

\section{Study of a High-Resolution PET System Using a Silicon Detector Probe}

\section{Karol Brzeziński}

\section{TESIS DOCTORAL UNIVERSITAT DE VALENCIA}

AÑ 2015

DIRECTORES DE LA TESIS

Magdalena Rafecas López

José F. Oliver Guillén

John Gillam

Departamento de Física Atómica, Molecular y Nuclear

Programa de Doctorado en Tecnologías para la Salud y el

Bienestar

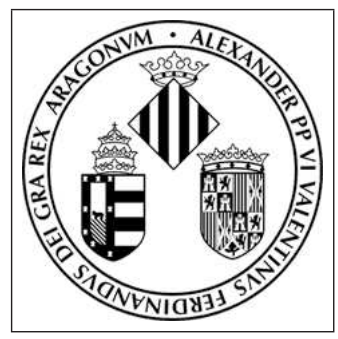




\section{Contents}

Summary \& Objectives $\quad 3$

$\begin{array}{lll}\text { I INTRODUCTION } & 7\end{array}$

1 POSITRON EMISSION TOMOGRAPHY 9

1.1 Introduction . . . . . . . . . . . . . . . . 9 9

1.2 Working Principles . . . . . . . . . . . . . 10

1.2.1 Physical Principles . . . . . . . . . . . . . 10

1.2.2 Sources of Degradation . . . . . . . . . . 14

1.3 Detectors . . . . . . . . . . . . . . . . . 18

1.3.1 Scintillation Detectors . . . . . . . . . 19

1.3.2 Alternate Detector Technologies . . . . . . . 26

1.4 Data . . . . . . . . . . . . . . . . 27

1.4.1 Types of Coincidence Events _ . . . . . . . 29

1.4.2 Spatial Resolution . . . . . . . . . . . 35

1.5 Image Reconstruction . . . . . . . . . . . . . . . 36

1.5.1 Analytic Reconstruction . . . . . . . . . . 37

1.5.2 Iterative Reconstruction . . . . . . . . . . . 40

1.6 Summary . . . . . . . . . . . . . . . . . . . 46

References .................... 46 
2 THE PET PROBE CONCEPT

2.1 Introduction . . . . . . . . . . . . . . . 55

2.2 Effect on resolution . . . . . . . . . . . . . 57

2.2.1 Photon acollinearity . . . . . . . . . 58

2.2 .2 Detector size . . . . . . . . . . . . 59

2.2.3 Acollinearity and Detector Size Combined . . . 63

2.2.4 Block effect . . . . . . . . . . . . 64

2.3 The Ring Insert Geometry . . . . . . . . . . . . . . . . 66

2.3.1 Working Principles . . . . . . . . . . 66

2.3.2 Existing systems . . . . . . . . . . . 67

2.4 The Probe Geometry . . . . . . . . . . . . 68

2.4.1 Working Principles . . . . . . . . . . 68

2.4 .2 Existing systems . . . . . . . . . . . . 68

2.4.3 Advantages and disadvantages . . . . . . . 69

2.5 Clinical applications of the PET probe . . . . . . 70

2.5.1 Breast Imaging . . . . . . . . . . . . . 70

2.6 Summary . . . . . . . . . . . . . . . 72

References .................... . . 72

3 PET PROBE SYSTEM DESIGN \& SIMULATION $\mathbf{7 9}$

3.1 Introduction . . . . . . . . . . . . . . . 79

3.2 The Clinical PET Scanner . . . . . . . . . . . . 80

3.2.1 Scanner Geometry . . . . . . . . . . . 80

3.2 .2 Timing and Energy . . . . . . . . . . . . 81

3.3 The PET Probe . . . . . . . . . . . . . . . . 81

3.3.1 Choice of Probe Material . . . . . . . . 82

3.3.2 Probe Design . . . . . . . . . . . . . . . 83

3.3.3 Timing and Energy . . . . . . . . . . . . . . 84

3.4 Simulating the Probe-and-Ring System . . . . . . . 86

3.4.1 Geometry .............. 86 
CONTENTS iii

3.4.2 Simulation Tools . . . . . . . . . . . . 86

3.5 Summary . . . . . . . . . . . . . . . . 89

References ...................... 89

II PREPARATORY WORK 93

4 EVENT CLASSIFICATION 95

4.1 Introduction . . . . . . . . . . . . . 95

4.2 Experimental Method . . . . . . . . . . . 96

4.2.1 Identifying Types of Events . . . . . . . . . 96

4.2.2 Simulation Experiment _. . . . . . . . . 97

4.3 Results. . . . . . . . . . . . . . 100

4.3.1 Effect of Coincidence Timing Window . . . . . 101

4.3.2 Effect of Probe LET . . . . . . . . . . . . 105

4.4 Discussion . . . . . . . . . . . . . . . 109

4.5 Conclusions . . . . . . . . . . . . . . 110

References . . . . . . . . . . . . . . . . 112

5 IMAGE RECONSTRUCTION TOOLS 115

5.1 Introduction . . . . . . . . . . . . . . . 115

5.2 Methods . . . . . . . . . . . . . 116

5.2 .1 Limited FOV . . . . . . . . . . . . . 116

$5.2 .2 \quad$ List-Mode ML-EM . . . . . . . . . . . . . . 118

5.2.3 Multi-Line Siddon . . . . . . . . . . . . . . 119

5.2.4 The Combined System Model . . . . . . . . . 122

5.2.5 Attenuation Correction . . . . . . . . . . . 124

5.2.6 Random and Scatter Correction . . . . . . . . 124

5.3 Results \& Discussion . . . . . . . . . . . . . . . . 127

5.3 .1 Limited FOV . . . . . . . . . . . . . . 127

5.3.2 Multi-Line Siddon . . . . . . . . . . . . . 128 
5.3.3 The Combined System Model . . . . . . . . . 130

5.3.4 Probe Attenuation Correction . . . . . . . . . 132

5.4 Conclusions . . . . . . . . . . . . . . . . . . 132

References ..................... 134

$\begin{array}{lll} & \text { III SIMULATION STUDIES } & 137\end{array}$

6 SYSTEM SPATIAL RESOLUTION 139

6.1 Introduction . . . . . . . . . . . . . . 139

6.2 Methods . . . . . . . . . . . . . . 140

6.2.1 Estimating Resolution . . . . . . . . . . . . 141

6.2.2 Initial Sinogram-Based Estimates . . . . . . . . 141

6.2.3 Variation Across the FOV . . . . . . . . . . . 142

6.2.4 Addition of Degradation Effects . . . . . . . . . 144

6.2.5 Resolution Phantom Study . . . . . . . . . 145

6.3 Results. . . . . . . . . . . . . . 145

6.3.1 Estimates from Sinograms . . . . . . . . . . 146

6.3.2 Testing for Convergence . . . . . . . . . . . . . 147

6.3.3 Variation Across the FOV . . . . . . . . . . . 148

6.3.4 Addition of Realistic Effects . . . . . . . . . . . 148

6.3.5 Resolution Phantom Study . . . . . . . . . . 151

6.4 Discussion . . . . . . . . . . . . . . . 153

6.5 Conclusions . . . . . . . . . . . . . . 158

References ...................... 159

7 IMAGE QUALITY 161

7.1 Introduction . . . . . . . . . . . . . . . . 161

7.2 SOR and Contrast Study . . . . . . . . . . . 162

7.2.1 Contrast Phantom . . . . . . . . . . . . . . 163

7.2 .2 Figures of Merit . . . . . . . . . . . 164 
7.3 Breast Imaging Study _ . . . . . . . . . . . . 166

7.3.1 Breast Phantom . . . . . . . . . . 166

7.3.2 Quantifying Lesion Detectability . . . . . . 168

7.4 Results. . . . . . . . . . . . . . . . . 169

7.4.1 SOR and Contrast Study . . . . . . . . . 169

7.4.2 Breast Imaging Study . . . . . . . . . . . . . 172

7.5 Discussion . . . . . . . . . . . . . . . . . 174

7.6 Conclusions . . . . . . . . . . . . . . 176

References ..................... 178

\section{EXPERIMENTAL SUPPLEMENT 181}

8 SILICON PET PROTOTYPES 183

8.1 Introduction . . . . . . . . . . . . . . . . . 183

8.2 Materials \& Methods . . . . . . . . . . . . . . . 185

8.2.1 Sources used . . . . . . . . . . . . 185

8.2 .2 The PET Scanner . . . . . . . . . . . 186

8.2.3 The Ring Insert Geometry . . . . . . . . . . . 186

8.2.4 The Probe Geometry . . . . . . . . . . . . . 188

8.2.5 Reconstructing the Data . . . . . . . . . . 192

8.3 Results \& Discussion . . . . . . . . . . . . . . . 195

8.3.1 2D Ring Insert Geometry . . . . . . . . . 195

8.3.2 3D Ring Insert Geometry . . . . . . . . . . . 198

8.3.3 3D Probe Geometry . . . . . . . . . . . . 198

8.3.4 Resolution Phantom: 2D Probe Geometry . . . 199

8.4 Conclusions . . . . . . . . . . . . . . . . 200 
V CONCLUSIONS

\section{SUMMARY IN SPANISH}

Estudio de un Sistema PET de Alta Resolución usando una Sonda de Detectores de Silicio 219

Introducción . . . . . . . . . . . . . . . . 219

Metodología . . . . . . . . . . . . . . . . . . . . . . . . . . . . . . . . . . . .

El sistema anillo-sonda . . . . . . . . . . . . . . 221

Reconstrucción de la imagen 222

Calidad de imagen . . . . . . . . . . . . . . . 223

Aplicación a mamografía PET . . . . . . . . . . . . . 226

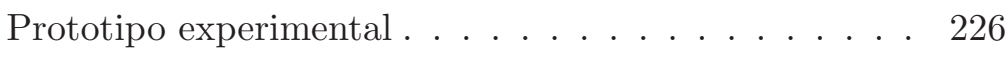

Resultados . . . . . . . . . . . . . . 227

Discusión y Conclusiones . . . . . . . . . . . . . 228

Referencias .................... 231 


\section{List of Figures}

1.1 Positron emission and annihilation. . . . . . . . . 13

1.2 Schematic diagram of a LOR. . . . . . . . . . . . 14

1.3 Intrinsic spatial resolution of a pair of face-to-face detectors. . . . . . . . . . . . . . . 17

1.4 The widening of VOR with increasing radial offset. . . 18

1.5 Schematic diagram of a scintillation detector. . . . . 20

1.6 Energy band structure of an inorganic scintillator crystal with an activator. . . . . . . . . . . 21

1.7 Schematic diagram of a PMT. . . . . . . . . . 23

1.8 Schematic diagram of a block detector. . . . . . . . . 24

1.9 Cross-section through a Si pixel. . . . . . . . . . 28

1.10 True and random coincidence. . . . . . . . . . . . . 30

1.11 multiple coincidence. . . . . . . . . . . . . . . . 32

1.12 Phantom-scattered coincidence. . . . . . . . . . . 33

1.13 2D PET data represented as projections. . . . . . . 38

2.1 Schematic diagram of a ring insert geometry. . . . . 56

2.2 Schematic diagram of a probe geometry. . . . . . . . 57

2.3 Resolution loss due to photon acollinearity as a function of position. . . . . . . . . . . . . . 59

2.4 The CRF as it varies along the length of the LOR. . . 61

$$
\text { vii }
$$


2.5 Resolution loss due to detector size as a function of position. . . . . . . . . . . . . 62

2.6 Resolution loss due to the combined effects of photon acollinearity and detector size as a function of position. 64

2.7 The effect of insert detector size on resolution for I-S events. . . . . . . . . . . . . . . 65

3.1 Initial experiments to characterize the Si detector timing response. . . . . . . . . . . . . . . 85

3.2 The proposed system with a Derenzo-like phantom. . . 87

4.1 Schematic diagrams of the types of probe coincidence events. . . . . . . . . . . . . . . . . 9 98

4.2 Undetected probe scatter. . . . . . . . . . . . 99

4.3 Position of the NEMA phantom relative to the probe. 101

4.4 Plots of the true coincidence rate. . . . . . . . . . . . 102

4.5 Plots of the random coincidence rate. . . . . . . . . . 103

4.6 Plots of the random fractions. . . . . . . . . . . . . . 104

4.7 Plot of the total probe coincidence rate. . . . . . . 105

4.8 Energy spectrum of detections in the probe. . . . . . 106

4.9 The effect of LET in the probe on the true probe coincidence rate and the TF. . . . . . . . . . . . . 107

4.10 Effect of LET in the probe on the object-scattered probe coincidence rate and the object SF. . . . . . . 108

4.11 Effect of LET in the probe on the random probe coincidence rate and the RF. . . . . . . . . . . . . 108

4.12 Plots of the relative contributions of trues, randoms and scattered events. . . . . . . . . . . . . 111

5.1 Limited FOV of the probe LORs. . . . . . . . . . 117

5.2 Sampling the response of a pair of detectors. . . . . . 121 
5.3 The 2D setup used for the probe attenuation demonstration. . . . . . . . . . . . . . 125

5.4 Sinograms showing the limited FOV of the probe in coincidence with the ring. . . . . . . . . . . 128

5.5 The effect of the probe's limited FOV in image space. 129

5.6 Effect of using the multi-line Siddon method on the ring sensitivity image. . . . . . . . . . . . . . 130

5.7 Effect of using the multi-line Siddon method. . . . . . 131

5.8 The value of $\beta$ measured at several probe offset positions.131

5.9 The effect of modeling attenuation due to the probe in the ring image. . . . . . . . . . . . . . 133

6.1 The grid of positions at which the point-spread function was calculated. . . . . . . . . . . . . . 143

6.2 The two orientations of the small Derenzo-like phantom relative to the probe. . . . . . . . . . . . 146

6.3 Sinograms of the four point sources. . . . . . . . . 147

6.4 2D PSFs of the ring and the probe data. . . . . . . 148

6.5 Results of convergence study. . . . . . . . . . . 149

6.6 Resolution in the direction perpendicular to the probe surface. . . . . . . . . . . . . 150

6.7 Resolution in the direction parallel to the probe surface. 151

6.8 Comparison of resolution from the simulated single column of point-sources. . . . . . . . . . . . 152

6.9 Images of the Derenzo-like phantom in configuration A. 153

6.10 Line profiles through the Derenzo-like phantom, in configuration A. . . . . . . . . . . . . . 154

6.11 Images of the Derenzo-like phantom in configuration B. 155

6.12 Line profiles through the Derenzo-like phantom in configuration B. . . . . . . . . . . . . . 156 
6.13 PSF for one point measured from the three data sets.

7.1 The phantom used for the image quality study and its position relative to the probe. . . . . . . . . . 164

7.2 The breast-and-torso phantom. . . . . . . . . 167

7.3 SOR at different distances from the probe, vs. iteration number. . . . . . . . . . . . . . 170

7.4 $C_{\text {hot } / \text { warm }}$ at different distances from the probe, vs. iteration number. . . . . . . . . . . . . . 171

7.5 $C_{\text {warm } / \text { cold }}$ at different distances from the probe, vs. iteration number. . . . . . . . . . . . . 172

7.6 Images of the breast region of the breast-and-torso phantom. . . . . . . . . . . . . . . 173

7.7 CRC and noise for the four central lesions. . . . . . . 174

7.8 CNR calculated for lesions of different sizes and different locations relative to the probe. . . . . . . . . 175

8.1 Experimental phantoms. . . . . . . . . . . 186

8.2 Photograph of the experimental setup. . . . . . . 187

8.3 2D ring insert geometry. . . . . . . . . . . . . . 189

8.4 3D ring insert geometry. . . . . . . . . . . . . . 190

8.5 Na source orientation. . . . . . . . . . . . . . . . 191

8.6 Derenzo-like phantom orientation. . . . . . . . . 193

8.7 Derenzo-like phantom reconstructed using BGO-BGO events only. . . . . . . . . . . . . . 196

8.8 Si-Si images. . . . . . . . . . . . . . . . . . 197

8.9 Na phantom reconstructed from the separate datasets. 203

8.10 FDG-filled vile reconstructed from the separate datasets.204

8.11 Na phantom reconstructed from Si-BGO data in the 3D probe configuration. . . . . . . . . . 205 
8.12 Images of the resolution phantom from the $2 \mathrm{D}$ probe data. . . . . . . . . . . . . . 206

8.13 Comparison of profiles through the resolution phantom. 207

14 El sistema PET con la sonda de silicio cerca de la superficie de un fantoma Derenzo. . . . . . . . . . . . . 222

15 La matriz de posiciones donde se midió la PSF. . . . . 224

16 El fantoma usado en estudios de contraste y SOR, en su posición relativa a la sonda. . . . . . . . . . . . 225

17 El fantoma antropomorfo en y posición relativa al escaner y la sonda. . . . . . . . . . . . . . . . . 227

18 Fotografía de prototipo experemental. . . . . . . . . 228

19 Comparación de la resolución espacial del Biograph y la del sistema sonda-anillo. . . . . . . . . . . . . . . 229

$20 C_{\text {hot } / \text { warm }}, C_{\text {warm/cold }}$ y SOR a varias distancias de la sonda en función de número de iteraciones. . . . . . . 230

21 Imágen de la mama sin usar la sonda e incluyendo la sonda en la simulación. . . . . . . . . . . . . . . . . . . 231

22 Imágenes del fantoma Derenzo reconstruidos con datos del anillo solamente e incluyendo datos de la sonda. 232 


\section{List of Tables}

1.1 Positron Ranges in Water for Radionuclides Used in PET 15

1.2 Properties of Selected Scintillator Materials Used in

Nuclear Medicine . . . . . . . . . . . . 22 


\section{Summary \& Objectives}

Clinical positron emission tomography (PET) is a medical imaging modality that provides three-dimensional information about the distribution of a tracer substance in a patient's body. Tracers are designed to follow specific metabolic pathways, giving PET the capability to provide functional rather than structural information. Although offering information complementary to that obtained using computed tomography (CT) and magnetic resonance imaging (MRI), clinical PET has a relatively low spatial resolution when compared to these other imaging modalities. There are practical limits on resolution in modern clinical scanners which utilize the standard PET ring geometry.

The use of high-resolution detectors, added within the scanner's field of view (FOV), has been proposed as a method of increasing the resolution of PET images. The "PET-probe" geometry is a case where one (or a stack of multiple) such detector(s) is placed close to a region of interest where higher resolution is desired. This work explores such a system, using a modern clinical PET scanner in coincidence with a probe consisting of a stack of Silicon detectors. The studies presented here quantify the improvement brought about by the probe and suggest a clinical application for the device. The system is studied at two levels: first, using Monte Carlo simulations of the system and second, 
by obtaining preliminary results from an experimental prototype.

The chapters of the thesis are grouped into five parts. The first part overviews the PET imaging technique and the PET probe principle before going into the details of the specific system on which this work is focused. The second part presents preparatory simulation studies of the system characteristics: an examination of event types is used to explore the effect of time and energy settings, and a study of the characteristics of the probe data is used to develop appropriate image reconstruction software. Further simulation studies, performed to characterize the capabilities of the probe system, are presented in part three. These studies quantify the improvement in image quality provided by the probe system with respect to that of the scanner alone. Any enhancement to the final image is expected to be localized, and therefore was studied as it varies over the FOV. The studies address image quality in terms of spatial resolution, contrast and spill-over ratio. In order to demonstrate the applicability of the system to PET mammography (PETM), simulations were conducted using a phantom which mimics a breast imaging scenario. In this context, lesion detectability was quantified in terms of contrast-to-noise ratio. Although here we focus on breast imaging, the proposed system could be used in other applications where high spatial resolution is desired in a determined region of interest. In the fourth part of this work, conclusions are presented as well as prospects for future investigation of the PET probe system. The fifth part of the thesis consists of a summary of the work performed, written in Spanish.

Also included as part of the thesis are studies performed using an experimental prototype of the PET probe system. Due to the preliminary nature of these studies they are not included in the main body of the work, but rather are presented as a supplement. The experimental studies present proof-of-concept support for the proposed system, 
while the experimental prototype acted as a physical testbed for the data processing and image reconstruction software developed within the dissertation. 
"thesis" - 2015/5/29 - 15:50 - page 6 - \#22 


\section{Part I}

\section{INTRODUCTION}




\section{Chapter 1}

\section{POSITRON EMISSION TOMOGRAPHY}

\subsection{Introduction}

Positron Emission Tomography (PET) is a medical imaging technique in the specialty of nuclear medicine. It makes use of positron emitting radionuclides to provide a three-dimensional (3D) map of metabolic processes inside the body. PET is widely used in clinical diagnosis as well as in pre-clinical studies. However, the focus of discussion throughout this work will be clinical PET. As a diagnostic tool, it is mainly used in oncology, cardiology and in the diagnosis of various brain diseases. By acquiring functional rather than structural information, PET is complementary to other imaging modalities. Modern PET scanners are combined with Computed Tomography (CT) systems to create the $\mathrm{PET} / \mathrm{CT}$ imaging technique, a powerful diagnostic tool that produces anatomical and functional information in a single co-registered image. Similarly, PET in combination with Magnetic Resonance Imaging (MRI) offers the advantages of both modalities in 
a co-registered PET/MRI image. In this chapter, a brief description of the underlying mechanisms of PET imaging is presented, from the positron emission itself to the formation of the final image.

\subsection{Working Principles}

Nuclear medicine imaging is based on the ability to trace a radioactive substance, relevant to the biological phenomenon being investigated, through the subject's body. The tracer compound, or radiopharmaceutical, follows certain biochemical pathways to localize to specific organs or cellular receptors. In PET imaging, the tracer is labeled with a positron-emitting radionuclide, usually incorporated into a specific pharmaceutically-active molecule which is injected into the body [1]. For example, the most commonly used radiopharmaceutical, fludeoxyglucose (FDG), is created by incorporating fluorine18 into deoxyglucose, an analogue of glucose. The concentrations of FDG imaged indicate regional glucose uptake and therefore tissue metabolic activity. There are a number of different radiopharmaceuticals employed in PET imaging, whose use depends on their chemical and biological properties and how they interact with the subject's metabolism.

Clinical PET is most often performed for static imaging that provides a $3 \mathrm{D}$ spatial map of radiopharmaceutical concentration. However, dynamic (4D) PET imaging is able to delineate both the temporal and spatial distribution of the tracer uptake, thus providing considerably more information about in vivo biology [2].

\subsubsection{Physical Principles}

The radiopharmaceutical accumulates in various regions of the body, ideally concentrating in certain tissues for which it is designed. Infor- 
mation about its distribution is transmitted to the PET scanner via gamma radiation. The following discussion explains the mechanisms of how the radionuclide produces this radiation, and how it is recieved by the PET scanner.

\section{Positron Emission}

A radionuclide is a nucleus with an excess number of either protons or neutrons. The imbalance makes the nucleus unstable and prone to changing its proton-neutron ratio to a more stable configuration by means of radioactive decay. If the nucleus has an excess of protons, it may decay through positron emission, also called $\beta^{+}$decay. In this process a proton $\left(p^{+}\right)$is converted to a neutron $(n)$, a positron $\left(\mathrm{e}^{+}\right)$and an electron neutrino $\left(\nu_{e}\right)$. The process can be represented schematically by

$$
p^{+} \rightarrow n+e^{+}+\nu_{e}
$$

The positron can be emitted at a range of energies, since the energy released during the nuclear reaction is shared by all of its products. Proton-rich radionuclides can also decay via electron capture. However, for PET imaging, radionuclides which favor positron emission are used.

\section{Annihilation}

After being ejected during $\beta^{+}$decay, the positron undergoes a series of inelastic collisions with atomic electrons as it passes through the tissue. The net distance it travels from the site of its emission is of the order of $10^{-2}$ to $10^{-1} \mathrm{~cm}$, depending on its energy, and is referred to as the positron range [3]. After losing most of its energy, the positron combines with an electron, its anti-particle, to form a system called 
positronium which resembles atomic hydrogen. Positronium is unstable and decays electromagnetically into two or more gamma rays depending on its state just prior to decay. The orthopositronium (oPs) and parapositronium ( $\mathrm{p}-\mathrm{Ps}_{\mathrm{s}}$ ) quantum states are determined by the alignment or nonalignment, respectively, of the spins of the electron and positron [4]. According to conservation laws, orthopositronium decays into odd numbers of photons and parapositronium into even numbers. Mean lifetimes of the two states are $142 \mathrm{~ns}$ for o-Ps and $125 \mathrm{ps}$ for p-Ps, making the even photon number decay much more common. Furthermore, within each of the two positronium states, the three- and two-gamma decay modes are the most common, with the probability of other modes quickly decreasing with photon count. The second most probable cases, the five- and four-gamma decay modes are negligible, having branching ratios of $1.0 \times 10^{-6}[5]$ and $1.439 \times 10^{-6}$ [6], respectively. As a result, the most common mode of positronium decay is the diagnostically useful two-gamma mode. In this case, in the rest frame of positronium, energy and linear momentum conservation require the two photons travel in opposite directions, each carrying half of the energy equivalent to the positronium mass, $511 \mathrm{keV}$ each. In the lab frame, however, the positronium is not completely at rest but rather contains a small amount of momentum. As a result, the trajectories of the two photons are not completely collinear. The formation and decay of positronium is commonly referred to as annihilation. The process of positron emission and annihilation is illustrated in figure 1.1, including acollinearity and positron range.

\section{Coincidence Detection}

The energy of the two annihilation photons, as they are called, gives them a high probability of exiting the body and being detected by the scanner, which consists of a ring of detectors surrounding the patient. 


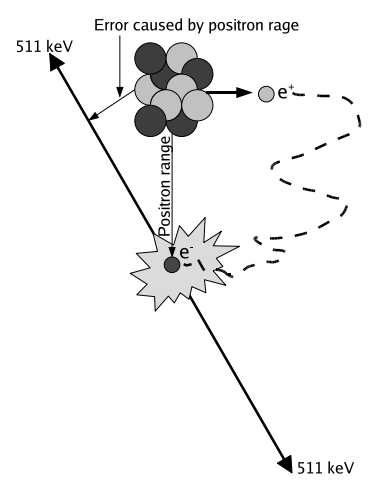

(a)

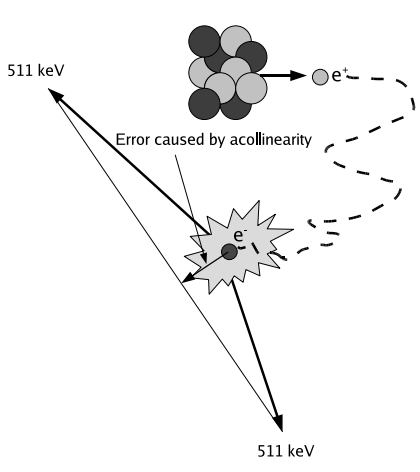

(b)

Figure 1.1: Schematic diagrams illustrating the process of positron emission and annihilation. A positron is emitted from a radionuclide and travels a short distance, losing energy, before annihilating with an electron. Upon annihilation, two back-to-back $511-\mathrm{keV}$ photons are emitted. For the LOR defined by the photon pair, the error due to positron range (a) is the distance from the emission site to the LOR. The error due to acollinearity (b) is the distance between the point of annihilation and LOR. Figures based on Phelps 2006 [3]

The near-simultaneous detection of both photons, originating from one annihilation, is called a coincidence event and forms the basis of PET imaging. Due to their approximately back-to-back trajectories, the photons are detected on either side of their point of origin, which must lie somewhere near the line joining the two detectors involved. This line is called the line of response (LOR). Because of the finite size of the two detectors, the shape of the region to which the annihilation location is confined is the tube-like volume connecting them, which can be called the volume of response (VOR). Figure 1.2 is a two dimensional representation of the concept of a VOR. 


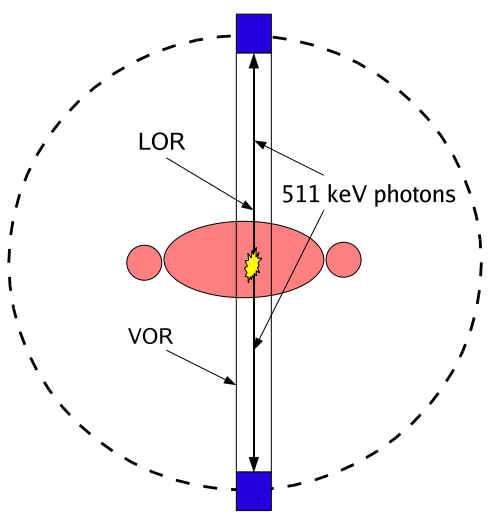

Figure 1.2: Schematic diagram of a LOR. The area between the two detectors defines a region within which the annihilation must have occurred.

\subsubsection{Sources of Degradation}

Several physical effects, related to the emission and detection of the annihilation photons, degrade the quality of the information available in a LOR. The following is a brief discussion of effects which reduce the precision or accuracy with which an LOR identifies the location of positron emission.

\section{Positron Range}

Positron range is the distance between the site where the positron is emitted and where it undergoes annihilation. The range is dependent upon the energy with which the positron is emitted, which varies from zero to a radionuclide-dependent maximum, and the material through which it travels. The quantity that directly affects the degradation of spatial resolution is the component of the net positron displacement vector that is perpendicular to the line defined by the annihilation 
Table 1.1: Positron Ranges in Water for Radionuclides Used in PET

\begin{tabular}{|l|l|l|l|}
\hline Radionuclide & $\mathrm{E}_{\beta}^{\max }(\mathrm{MeV})$ & FWHM $(\mathrm{mm})$ & FWTM $(\mathrm{mm})$ \\
\hline${ }^{11} \mathrm{C}$ & 0.970 & 0.188 & 1.86 \\
\hline${ }^{13} \mathrm{~N}$ & 1.19 & 0.282 & 2.53 \\
\hline${ }^{15} \mathrm{O}$ & 1.72 & 0.501 & 4.14 \\
\hline${ }^{18} \mathrm{~F}$ & 0.635 & 0.102 & 1.03 \\
\hline
\end{tabular}

*Based on data from Levin et al. [7].

photons. This distance is referred to as the effective positron range. The distribution of this range about the point of annihilation is characterized by a sharp peak with long tails, rather than a Gaussian [7]. Therefore, in addition to the full width at half maximum (FWHM) of the distribution, the full width at one-tenth maximum (FWTM) should be given to characterize the distribution. The maximum energies and effective positron ranges in water for several radionuclides are presented in table 1.1 .

\section{Acollinearity}

The magnitude of photon acollinearity is independent of the radionuclide used because the positron's final and initial energies are independent. However, the extent to which acollinearity impacts the data depends on scanner diameter since the further the photons have to travel before being detected, the larger the distance separating the line between detection locations and the site of annihilation. At the centre of two detectors separated by a distance $D$, the FWHM of the blurring profile due to acollinearity is $0.0022 D[8]$.

\section{Detector size}

The size of the detectors determines the precision with which an annihilation event is located within the VOR, at any given position along its length. This precision is called the intrinsic resolution of a detec- 
tor pair and is described by the coincidence response function (CRF). The CRF is the probability that a point source, which is moved perpendicular to the LOR corresponding to a detector pair, is detected as a coincidence event. PET scanners typically use identical detectors around the ring, so the CRF of a pair of any of these is symmetric about the midpoint between the two. For LORs near the centre of the FOV, where the detectors are face-to-face, the CRF has the shape described in figure 1.3. It forms a triangle at the centre of the VOR and becomes rectangular near the ends [3]. At intermediate positions the CRF is trapezoidal in shape, with a plateau whose width is equal to the distance between two lines joining opposing sides of the two detectors. The width of the base of the CRF is the same as the detector width $w$. Figure 1.3 shows the CRF at different positions for a pair of face-to-face detectors of the same size. The spread of the CRF is given by its FWHM and is a measure of intrinsic resolution. Resolution is highest when the CRF is triangular, in the centre, and degrades as the function spreads out to be trapezoidal and, finally, rectangular. For LORs located away from the centre of the FOV, where the detector pairs are not directly facing each other, the shape of the CRF varies due to the effect of the depth of photon interaction in the detector and becomes asymmetric.

\section{Depth of Interaction}

In order to stop 511-keV annihilation photons efficiently, PET detectors are designed to be 2 to $3 \mathrm{~cm}$ thick, corresponding to 2 to 3 times the attenuation length of the detector. When a photon enters a detector at an oblique angle, uncertainty regarding the depth of interaction (DOI) causes a degradation of intrinsic resolution known as parallax. The effect of DOI uncertainty is illustrated in figure 1.4 for the case of a circular scanner. For LORs away from the centre of the FOV, the ef- 


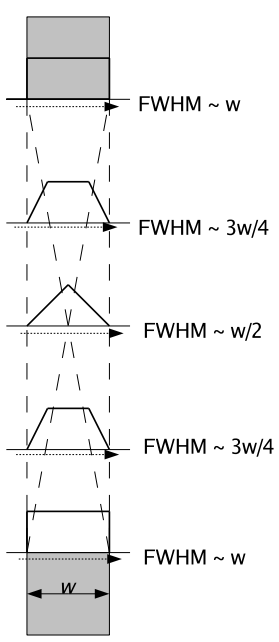

Figure 1.3: Intrinsic spatial resolution of a pair of face-to-face detectors. The width of the base of the CRF is equal to the width $w$ of the detectors. It is triangular at the midplane, with $\mathrm{FWHM}=w / 2$. Moving towards the detectors it becomes trapezoidal and eventually rectangular at their surfaces. Figure based on Phelps [3]

fective width of the detectors is increased due to their inclination with respect to the LOR. This causes a widening of the VOR (spreading of the CRF) and therefore loss of resolution [9]. The effective width is given as:

$$
w^{\prime}=w \cos \theta+l \sin \theta
$$

where $w, l$ and $\theta$ are the width, length and inclination angle of the detector. In such LORs, the modified value $w^{\prime}$ serves as the width of the base of the CRF. The FWHM is modified by the multiplicative factor $\cos \theta+(l / w) \sin \theta$. In practice, this factor is always larger than 1 and its effect is to increase the FWHM, thereby degrading resolution. 


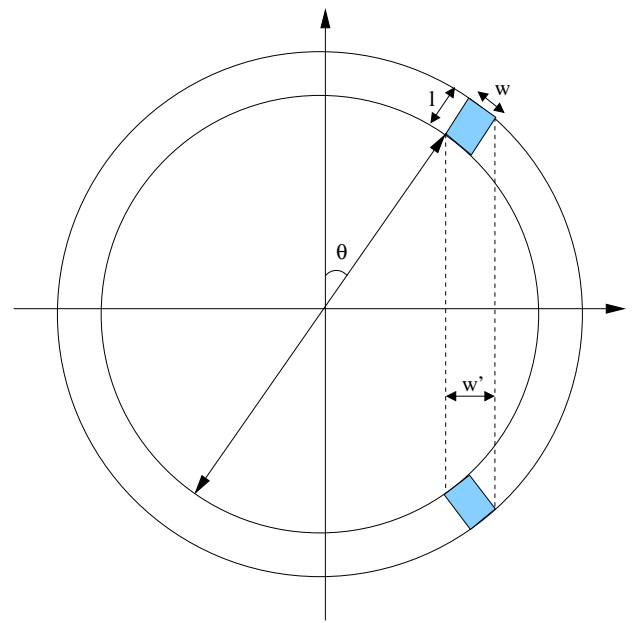

Figure 1.4: The widening of VOR with increasing radial offset is due to increased effective width of the detectors. The annihilation event could have occurred anywhere between the two dashed lines. Figure based on Cherry et al. [9]

The CRF is further modified due to the effect of varying detector thickness across the radial width of the VOR, the function increasing where the detectors are thicker (see figure 1.4). For a scanner with 4-mm-wide detectors and 80-cm diameter, the parallax effect causes a $40 \%$ loss of resolution from the centre of the FOV to a point offset by $10 \mathrm{~cm}[9]$.

\subsection{Detectors}

A clinical PET scanner is made up of radiation detectors which surround the patient in order to simultaneously detect annihilation photon pairs on opposite sides of the body. For this purpose, detectors are placed around the patient, typically arranged in several axially-offset 
rings of about $1 \mathrm{~m}$ diameter and several centimeters in axial extent.

A number of properties make a detector suitable for use in PET scanners [3]. One of the most important of these is spatial resolution, the ability of a detector to determine, with precision, the location of the photon interaction within its sensitive material. Detector resolution is directly related to that of the reconstructed image. Another important property is high detector photoabsorption efficiency for 511-keV photons, that is the fraction of those photons incident on its surface that are photoelectrically absorbed. High efficiency allows for more photon pairs to be collected which leads to a higher signal-to-noise ratio in the images. Good timing resolution, the precision of a detector's temporal response, allows the system to accurately group detected photons into annihilation pairs according to the time of detection. Finally, good energy resolution, the precision with which a detector can measure deposited energy, is essential for discarding unwanted photons which have lost energy after having undergone Compton scattering in the the body (see section 1.4.1). Below is an examination of the types of detectors used in PET.

\subsubsection{Scintillation Detectors}

The main type of radiation detector used in clinical PET scanners is the scintillation detector. This device consists of a scintillator material, which offers a high cross-section to $\gamma$-ray photons, converting their deposited energy to visible light, and a light sensor, which converts the visible light into an electrical signal. The basic process is described schematically in figure 1.5. First, the scintillator material is excited by the interaction of a high-energy photon. In organic scintillator materials, the excitation is an inherent molecular property, while in inorganic materials it occurs in their crystal structure [10]. Upon de-excitation, a portion of the energy is released as visible and UV 


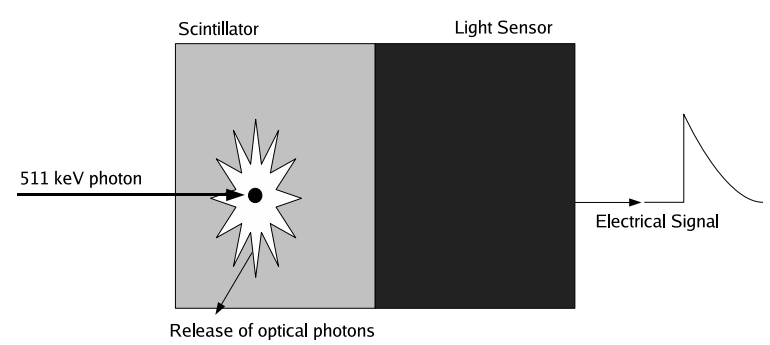

Figure 1.5: Schematic diagram of a scintillation detector. When a photon interacts in the scintillator, light is emitted isotropically. Part of it arrives at the light sensor which produces an electrical signal proportional to the photon energy.

light, the amount of which is proportional to the energy deposited by the photon. For a single 511-keV photon, the number of light photons released is in the thousands or tens of thousands [10]. A part of this light arrives at the surface of the light sensor and is converted into an electrical signal.

The most common organic scintillators are in liquid form. They are used in medical applications for investigating biological samples using non-penetrating radiation. Liquid organic scintillators are inefficient for penetrating $\gamma$-rays and have relatively low light output [9]. For PET detectors, inorganic scintillators are a much better choice due to their higher cross-section at $511 \mathrm{keV}$, while remaining transparent at the scintillation photon energies. The scintillation process in these materials depends on the energy states determined by their crystal lattice. In the pure scintillator, electrons can be found in the valence band, bound at lattice sites, or in the conduction band, with sufficient energy to be free to migrate through the crystal. They can never be found in the forbidden band, at intermediate energies. The absorption of sufficient energy from a $\gamma$-ray interaction may excite an 


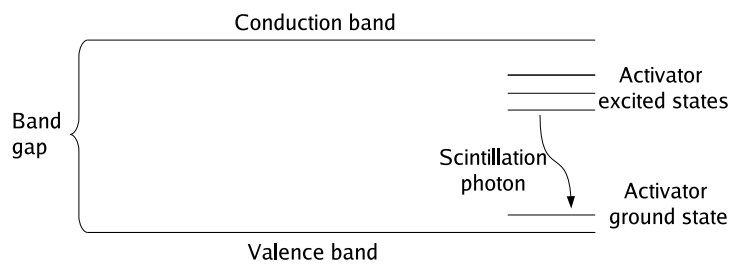

Figure 1.6: Energy band structure of an inorganic scintillator crystal with an activator. Figure based on Knoll [10].

electron in the valence band into the conduction band. The electron returns to the valence band by emitting a photon. However, in the pure crystal, this mechanism is inefficient and generally the photons released are not in the visible spectrum. For this purpose, impurities, called activators, are introduced into the scintillator material and alter the normal energy band structure. Energy states are created in the forbidden band, providing the excited electron with an alternate mode of de-excitation, one that releases photons in the visible light spectrum [10]. The mechanism is illustrated in figure 1.6.

Besides sensitivity, light yield is also an important property of the scintillator since it influences the detector's spatial and spectral (energy) resolutions. Localization of the point of interaction in the detector depends on the distribution of light arriving on the light detector surface. Higher light output in the scintillator lowers the statistical fluctuation of its intensity at the sensor and in turn increases precision of the position measurement. This is also true for the precision of energy measurement, whose value is proportional to the amount of light detected and is used to identify scattered photons. Other important factors which determine the suitability of scintillators for use as PET detectors are: density, for providing sufficient stopping power; decay 
Table 1.2: Properties of Selected Scintillator Materials Used in Nuclear Medicine

\begin{tabular}{|l|l|l|l|l|l|}
\hline Property & NaI $(\mathrm{Tl})$ & BGO & LSO $(\mathrm{Ce})$ & GSO $(\mathrm{Ce})$ & CsI(Tl) \\
\hline Density $\left(\mathrm{g} / \mathrm{cm}^{3}\right)$ & 3.67 & 7.13 & 7.40 & 6.71 & 4.51 \\
\hline Decay time $(\mathrm{nsec})$ & 230 & 300 & 40 & 60 & 1000 \\
\hline Photon yeild (per keV) & 38 & 8 & $20-30$ & $12-15$ & 52 \\
\hline Index of refraction & 1.85 & 2.15 & 1.82 & 1.85 & 1.80 \\
\hline
\end{tabular}

time, which, together with light yield determines timing accuracy; and the index of refraction, which indicates the amount of generated light that makes it to the light detector [3]. The properties of some inorganic scintillators used in nuclear medicine applications are summarized in table 1.2. In this work, Bismuth Germanate (BGO) and Lutetium Oxyorthosilicate (LSO) are used, in experiment and simulation, respectively. In the experimental section, photomultiplier tubes (PMTs) are used as light sensors.

\section{Photomultiplier Tubes}

PMTs are commonly used to convert light emitted by the scintillator material into an electric signal. Scintillation light incident on the PMT surface enters through a glass window and excites a photocathode, liberating photoelectrons. These are accelerated through a vacuum chamber under high voltage to the first in a series of charged electrodes called dynodes. At each consecutive dynode, one electron releases 3 to 4 secondary electrons, creating an avalanche, which, after passing through 10 stages of amplification, for example, would contain on the order of $10^{6}$ electrons [3]. In the period of a few nanoseconds, a small current, on the order of milliamps, is created at the PMT anode. A schematic representation of this process is shown in figure 1.7. 


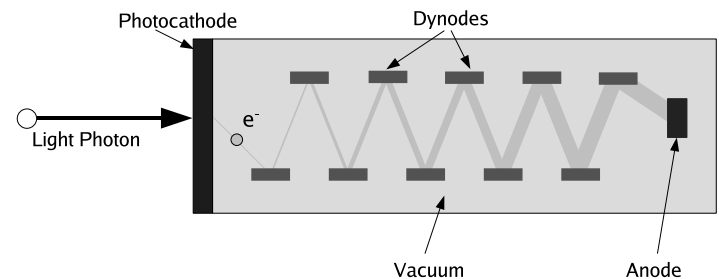

Figure 1.7: Schematic diagram of a PMT. A single photon from the scintillation light is incident on the photocathode and releases an electron. The electron hits a dynode releasing secondary electrons which are accelerated through a series of further dynodes, each at a higher voltage, releasing further electrons resulting in a small current at the anode.

\section{Block Design}

Most scintillation detectors used in clinical PET scanners follow the block design. A block detector consists of a scintillator crystal, a few centimeters deep and usually with a square or rectangular surface, measuring a few centimeters on each side. The scintillator is segmented into small detector elements, whose sides measure on the order of millimeters. The cuts which segment the detector's front surface are of varying depth and do not pass all the way through the scintillator, but stop short of reaching the PMTs coupled to its back surface (usually four). The amount of scintillation light received by each PMT is proportional to the position at which the high-energy photon interacted. The detector elements are optically isolated with reflective material filling the cuts. This geometry is shown schematically in figure 1.8, which includes side and top views of a simplified block detector. The depth of the cuts allows partial light-sharing which makes it possible to determine the detector element where the interaction occurred, given a certain distribution of scintillation light 


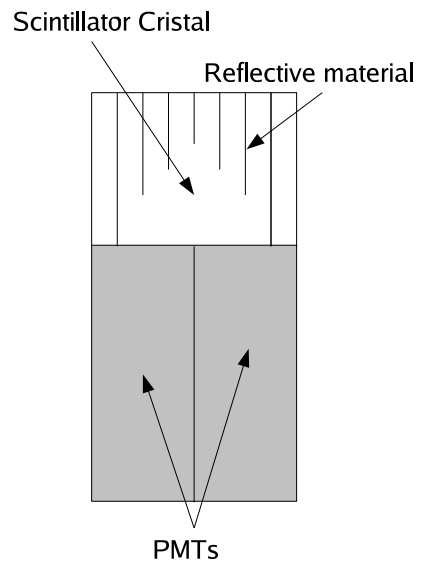

(a)

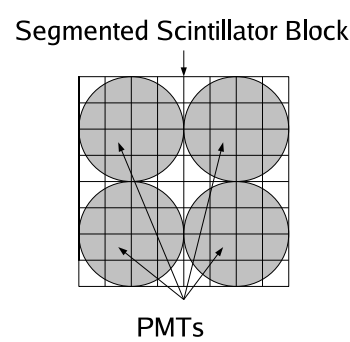

(b)

Figure 1.8: Schematic diagram of a block detector including a) a side view and b) as seen from the inside of the scanner, with the PMT locations on the other side of the crystal also visible.

arriving at the PMTs and, consequently, a certain combination of PMT signals.

In this work, the block design is utilized in the scintilation detectors of the simulated and experimental PET rings.

\section{Beyond the Block Design}

Advances in detector technology are changing the design and application of scintillator detectors in PET imaging. Here we briefly describe some of the most important improvements made to PET detectors based on the scintillator design, mostly in the pre-clinical phase of development. Improvements in electronics and PMT performance have produced faster detectors which allow for time-of-flight PET, a technique which utilizes the difference in detection times of the two an- 
nihilation photons for better localization of the event along the LOR [11]. In addition to timing, PMTs have improved in terms of the bulk of the devices and, as a result, increased the number of PMT channels available for reading a crystal array. Devices with multiple dynode chains inside a common vacuum envelope are available, each channel effectively an independent PMT. These devices decrease dead space in the light detector and increase the ratio of PMTs to crystals [12].

Substantial work has also been done on developing alternatives to PMTs. The use of various forms of solid-state photodetectors has provided many benefits, including: one-to-one photodetector-to-crystal coupling for small crystal sizes, multiple-surface readout of the scintillator, more compact design and operability in magnetic fields [12]. Silicon PIN diodes have been studied as light detectors for PET applications [13]. These devices offer the above-stated advantages but have low gain and poor timing resolution. The avalanche photodiode (APD), on the other hand, offers improved gain and timing performance. APDs are available as arrays of sensors as well as positionsensitive planar devices (PS-APD) where signals from the four corners of the device are used to determine the position of an event [12]. The Geiger-mode APD, or silicon photomultiplier (SiPM), is another widely studied photosensor in novel PET research [14, 15]. These devices consist of series of APD micro-cells, each an independent Geiger-mode detector capable of digital (on/off) reading. Light photons released in the scintillator interact in multiple micro-cells at once. SiPMs are capable of excellent timing performance and with high-output, fast scintillators can achieve 250 - 500-ps resolution. The development of the new photosensors has opened up the possibility of one-to-one coupling and enabled the development of new scintillation detector designs beyond the block model.

Another area of development in scintillation detector design is that 
of DOI determination capabilities, for reducing parallax with little cost to sensitivity. One of the first approaches to achieving DOI resolution was the phoswich technology, which uses scintillators with different decay times at different depths of the detector and a pulse shape discriminator (PSD) to determine the layer in which the interaction took place $[16,17]$. Limitations of this approach include high cost of fabrication, poor timing resolution and high scatter. Several solutions to these problems based on the PSD method have been proposed [12]. Non-PSD methods have also been employed. One of the first such attempts used two radial layers of LSO detectors, each read by a its own APD unit [18]. An alternative method used offset layers of scintillator crystals of the same material read by position sensitive PMTs capable of decoding each layer [19]. Another widely studied method has been to place photosensors on either end of the scintillation crystal and using the fraction of light received by each to determine the DOI [20]. A third technique is to use crystal pairs with a depth-varying absorber interface. If each crystal is individually read out, the fraction of light from each provides DOI information [21].

\subsubsection{Alternate Detector Technologies}

Although scintillation detectors are standard in PET systems, there has been much interest in alternate materials, with the goal of achieving higher spatial resolution than that imposed by the current state of scintillator detector development. To this end, work has been done using wire chambers [22] or similar designs based on scintillator sheets, layers of scintillator fibers [23] or resistive plate chambers (RPC) [24]. These designs provide high spatial resolution and good timing performance to various degrees but have poor energy resolution and are generally complex to fabricate and apply to PET scanners.

Another alternative to scintillator detectors widely investigated in recent years is the use of solid-state detectors. Work has been 
done using various semiconductor materials such as silicon ( $\mathrm{Si})[25,26]$ and high purity germanium (HPGe) [27] as well as the compounds cadmium telluride (CdTe) [28] and cadmium zinc telluride (CdZnTe or CZT) [29]. For reasons which will become apparent in chapter 3, Si detectors are of particular interest to this work. A brief description of these is included below.

\section{Silicon Detectors}

One of the most mature semiconductor materials for radiation detection is Silicon $(\mathrm{Si})$ and its potential applicability to PET imaging has already been demonstrated [25]. A typical detector consists of a thin slab of $\mathrm{Si}$, usually about $1 \mathrm{~mm}$ thick, with a potential difference applied across its thickness creating a depleted region. A photon traversing the Si has a certain probability of interacting in the material and liberating an electron. The electron then loses energy as it travels through the $\mathrm{Si}$, creating electron-hole pairs along its path [10]. The electrons and holes drift in the applied electric field to the cathode and anode, respectively. Figure 1.9 shows a schematic diagram of the basic working mechanism of a Si detector. Although Si detectors have relatively poor efficiency at $511 \mathrm{keV}$ (2\% per mm, with 1 photoabsorption for 499 Compton interactions), several layers of the detector material can be used to increase stopping power. Such a layered design has been applied to Compton camera technology for PET imaging [30].

\subsection{Data}

To exclude photons which have altered their path while undergoing Compton-scattering in the patient, only those photons which deposit their known emission energy in the detector via the photoelectric effect 


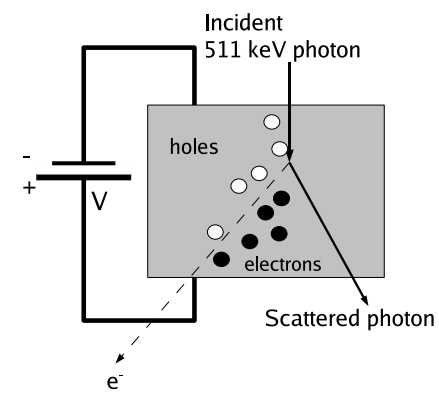

Figure 1.9: Schematic diagram of a cross-section through a Si pixel. An annihilation photon undergoes Compton scatters in the Si material, liberating a Compton electron. The electron traverses the Si creating electrons (in black) and holes (in white) which move towards the electrodes.

should be considered as usable events. However, the limited energy resolution of a detector must be considered when identifying a 511-keV photoelectric energy deposit. In order to minimize sensitivity loss, an energy acceptance window is defined that includes photons which deposit energy within a range around $511 \mathrm{keV}$. The width of the energy window is adapted to the energy resolution of the system. Typical values are 350-650 keV, but this range can be made narrower as energy resolution is enhanced. Limited timing resolution and variable photon path-lengths cause the detection of the annihilation photons to occur within a small time interval rather than simultaneously. Time resolution is considered when defining the duration of a coincidence detection. To optimize sensitivity, the coincidence timing window (CTW) is adjusted accordingly (usually to twice the time resolution). 


\subsubsection{Types of Coincidence Events}

The finite energy and timing resolutions of the PET detectors, and the corresponding timing and energy windows used for coincidence sorting, result in a variety of types of coincidence events. A long CTW results in an increase in those coincidences which involve photons from uncorrelated annihilation events, as well as those involving more than two photons. A large energy window causes an increase in scattered events being accepted. The following is a brief introduction to the different types of coincidence events measured during a PET scan.

\section{True Coincidences}

A true coincidence is the detection of two photons, originating from the same annihilation event, within the CTW. If, in addition, the detected photons do not interact in the patient or other part of the scanner, these events provide information about the activity distribution being imaged. Sensitivity to true coincidences should be maximized to obtain optimal image quality. Figure 1.10 a) shows a schematic diagram of this type of event.

\section{Random Coincidences}

A random coincidence, also called an accidental coincidence, is the detection within the CTW of two photons which originate from different annihilation events. Figure $1.10 \mathrm{~b}$ ) shows a schematic diagram of a random coincidence. These events provide no information about the activity distribution being imaged and add an undesired background to the reconstructed images.

Random coincidences can be quantified using several methods. The most common way of estimating the randoms rate in a particular LOR is using the delayed coincidence channel method [31]. The 


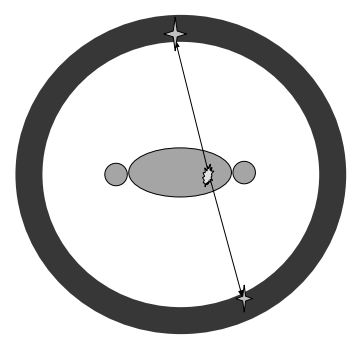

(a)

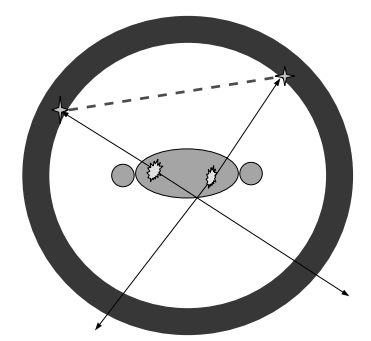

(b)

Figure 1.10: Schematic diagrams of a) a true and b) a random coincidence. In the case of the true coincidence, the two detected photons originate from the same annihilation event, which occurs on the LOR (up to the limit of acollinearity). In the random case, the two detected photons originate from two separate annihilation events. Here, the LOR (dotted line) is unrelated to any point of annihilation.

method consists of delaying the signal from one of the detectors by a time significantly greater than twice its timing resolution $\tau$, then sorting for coincidences using the standard CTW of $2 \tau$. The resulting delayed coincidence events cannot contain any trues, since a detected single can only be paired with one from another event. The coincidences formed represent a good estimate of the randoms contribution to the prompt data. The advantage of this method is that the randoms estimate for a certain LOR is computed using the delayed coincidence rate in the same LOR, having identical dead-time properties. On the other hand, the statistics in the delayed channel estimate are on the order of those in the prompt data (or lower) and are a source of statistical noise.

An alternative method for estimating the randoms contribution to a given LOR is using the singles rate (SR) approximation. Using this method, the randoms rate $\mathrm{N}_{R}$ is given by 


$$
N_{R}=2 \tau N_{1} N_{2}
$$

where $\mathrm{N}_{1}$ and $\mathrm{N}_{2}$ are the single detection rates in each individual detector in the pair. Since the SR is much greater than the coincidence rate, applying the correction does not introduce as much statistical noise into the data as the delayed coincidence channel method. As its main drawback, the SR method has been shown to systematically overestimate the randoms rate [32]. However, it is one of the most widely used estimations methods and continuing research strives to overcome this main limitation.

The randoms estimates are used to reduce the degrading effect of these events on the reconstructed image. An estimate may be subtracted from the prompt coincidence data as they occur, or stored for subsequent use in the image reconstruction algorithm. If online data correction is used, subtracting the randoms estimate leaves corrected data with degraded statistical quality. On the other hand, using the estimate to compensate for randoms in the process of image reconstruction requires an estimate with low statistical noise. For this purpose, the singles-rate method is an appropriate choice, and was used wherever randoms compensation was performed in this work.

\section{Multiple Coincidences}

Besides randoms, a long CTW may also increase the preponderance of multiple coincidences, where three or more single events are detected in the same CTW, an effect known as pileup. As the CTW is extended, the more probable multiple coincidences become, replacing what otherwise may have been true coincidences. In the case of a coincidence event involving three singles, there are two or three possible LORs and identifying which (if any) corresponds to a positron emission is non-trivial. These events are generally discarded. One pos- 


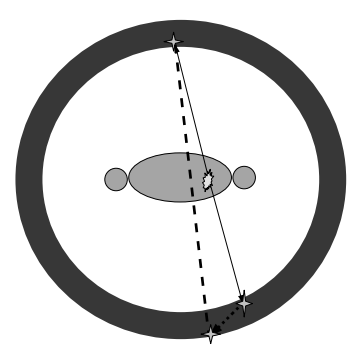

(a)

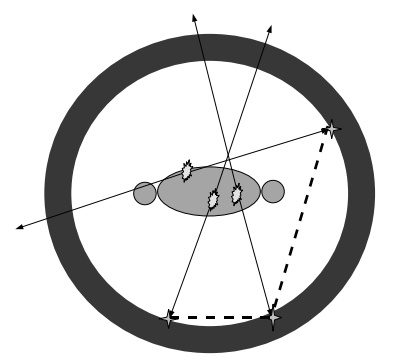

(b)

Figure 1.11: Schematic diagram of a multiple coincidences involving a) ICS and b) a random coincidence. Three events are detected within the CTW, creating multiple possible LORs: two in the case of ICS and three in the random case. In each case one of the LORs is related to a point of annihilation and the others are not (dashed lines). The trajectory of the crystal-scattered photon in a) is shown as a dotted arrow.

sible mechanism for multiple coincidences occurs when one (or both) of the annihilation photons undergoes inter-crystal scatter (ICS) and produces individual signals in each of the two detectors in which it interacted. Although the low-energy threshold (LET) of the energy acceptance window is designed to reduce the frequency of this type of event, the finite energy resolution of the detectors creates a possibility for their occurrence. Figure 1.11 a) shows a schematic diagram of a multiple coincidence event involving ICS. Another possibility is random multiple coincidence, which occurs when three or more uncorrelated 511-keV photon are detected within the CTW. A long CTW increases the likelihood of the occurrence of this type of event, which is described in Figure $1.11 \mathrm{~b}$ ). 


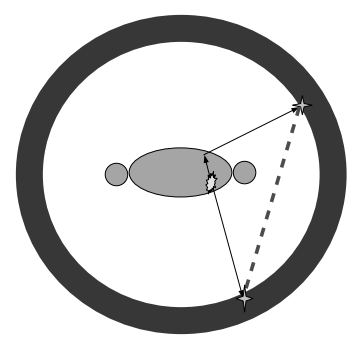

(a)

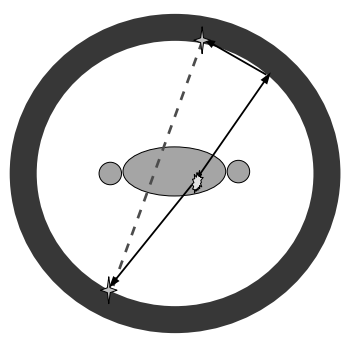

(b)

Figure 1.12: Schematic diagram of a phantom-scattered coincidence. Here, one of the photons undergoes scatter in the phantom before being detected, thereby displacing the LOR (dotted line) away from the point of annihilation. Schematic diagram of inter-crystal scatter, were an annihilation photon initially scatters in one crystals and is subsequently detected in another. The LOR (dotted line) is displaced away from the point of annihilation.

\section{Object-Scattered Events}

Before reaching the detectors, annihilation photons may undergo scatter in the object being imaged. As they are scattered, depositing a fraction of their energy in the subject, these photons change their trajectory, thereby displacing the LOR away from their point of emission as described in figure 1.12 a). Events involving object-scattered photons reduce image resolution since they contain information from a displaced LOR. These events can be partially removed from the data by employing a LET in the detectors. Scattered photons with energy below the LET will not be accepted. However, some low-anglescattered photons will have energy above the LET since the threshold must be low enough to account for energy resolution in the detectors.

The most commonly used scatter correction schemes include the tail-fitting technique $[9,33,34]$ and model-based scatter simulation methodologies [35, 36]. 
The tail-fitting method consists of fitting a function (most often a Gaussian) to the scatter tails immediately outside the object at each projection. This method is well suited for cases where the activity and scatter medium occupy only a small portion of the FOV and follow a fairly uniform distribution, such as in the case of brain imaging.

The more accurate, model-based scatter simulation algorithms use the non-corrected emission image and an attenuation map obtained from a transmission image together with a model of the scanner geometry to estimate the contribution of scattered events at each LOR. One implementation of such a methodology, the Single Scatter Simulation algorithm [36] has been used for scatter correction in the PET insert device developed by the Washington University group [37].

A technique based on Monte-Carlo modeling of the imaging system, together with activity and attenuation distributions, has also been proposed [38]. Using this method, the history of each generated photon is followed and a scattered coincidence distribution is determined.

\section{Undetected Scatter in Ring Detectors}

Before being detected, some annihilation photons may undergo scatter in the sensitive detector material but, because they deposit an amount of energy below the LET in that detector, not be recorded. Such is the case with undetected ICS, where the photon interacts in the first crystal, depositing an amount of energy below the LET, thus generating no signal, and is absorbed in a second detector element. As in the case of phantom scatter, the change in trajectory these photons undergo causes a reduction of the resolution information they carry. Figure $1.12 \mathrm{~b}$ ) shows a schematic diagram of an event involving undetected scatter in the detectors. 


\subsubsection{Spatial Resolution}

The spatial resolution of a medical imaging system is often measured as the extent of its point spread function (PSF), that is of the response of the system to a high-contrast point-like source distribution. In PET, the PSF is the three-dimensional image obtained when imaging a point source. Image resolution is usually expressed as the FWHM of the PSF, the closest distance that two Gaussians of this width can be brought together and still exhibit two separate peaks. The physical effects contributing to the reconstructed image resolution of a conventional scanner using cylindric geometry have been identified as: the detector crystal width and photon acollinearity, which contribute to the intrinsic resolution, and source size and the tomographic reconstruction process [39]. Though of some importance in small-animal PET, the effect of positron range is negligible in clinical scanners compared to other factors. The contributing factors can be expressed in an empiric formula proposed by Moses and Derenzo [40]:

$$
R_{\text {sys }}=1.25 \sqrt{(w / 2)^{2}+(0.0022 D)^{2}+s^{2}+b^{2}}
$$

In this equation $R_{\text {sys }}$, the FWHM (in mm) of the PSF, is expressed as a function of the crystal width $w$, the scanner diameter $D$ and the effective source size $s$, which includes positron range. The factor of 1.25 is due to the image reconstruction process and may vary slightly depending on the algorithm used. The variable $b$ represents the socalled "block effect", the degradation caused by events mispositioned in the block detector. The block effect is in fact the combined effect of several phenomena which occur inside a block detector. These include the multiple interactions of an annihilation photon in the scintillation crystal array, light transport in the scintillator as a function of the depth of interaction, electronic noise in the PMTs, undersampling of 
the image by the detectors, errors in the crystal identification matrix, and the discrepancy between the effective crystal location and its physical location [41]. A typical value for $\mathrm{b}$ is $2 \mathrm{~mm}$ [40].

The spatial resolution of a PET system also influences its ability to accurately represent radioactivity concentrations in structures smaller than $2 \times$ FWHM. Due to the so-called partial-volume effects the radioactivity concentration in objects of this size will be either underestimated (for objects with higher activity than the background) or overestimated (for objects with lower activity than the background).

\subsection{Image Reconstruction}

PET image reconstruction uses mathematical algorithms to convert coincidence detection data into a 3D map of the distribution of positronemitting radiophamaceutical inside the scanned object. In this sense, image reconstruction becomes an inverse problem for which we attempt to determine an unknown activity distribution $\mathbf{f}$ given projection measurements $\mathbf{p}$ and a linear operator $\mathbf{A}$ that describes the imaging process as

$$
\mathbf{p}=\text { Af. }
$$

Expressed in this way, the problem is linear since the projection data consists of detector-pair counts which are proportional to the total activity along the corresponding LOR, essentially line integrals through the activity distribution in the object. In reality, the presence of noise in the imaging problem is expressed as an extra term in the above equation.

There are two general approaches for solving the problem. Analytic reconstruction provides an exact solution to the inverse problem in the absence of noise, using a mathematical transform to relate the 
line integrals to activity distribution. The methods are linear and therefore robust. The second common approach, iterative reconstruction, attempts to determine the distribution most consistent with the measurements using a model of the system, which can include noise, and a criterion that measures the correctness of the fit. The following is a brief overview of the two methods, with a focus on one specific example of an iterative algorithm, ML-EM, which is used throughout this work.

\subsubsection{Analytic Reconstruction}

The analytic method, based on the inverse Radon transform, has historically been the most commonly used for image reconstruction in PET. Among the various analytic algorithms, filtered backprojection (FBP) might be considered the general standard. However, in this discussion we focus on iterative methods and FBP is presented here only briefly. This is done for the sake of completeness and also to introduce important concepts, which will be useful in the discussion throughout this work.

\section{Projection data}

From measured data, all parallel line integrals can be grouped together to form projections of the object from various angles. This representation of the distribution is a discrete approximation of its Radon transform. For simplicity, in the following discussion we concentrate on the $2 \mathrm{D}$ case. Figure 1.13 a) shows a projection $p(S, \theta)$, of a $2 \mathrm{D}$ activity distribution $f(x, y)$. The projection data can be discretized and organized into a 2D histogram, called a sinogram, whose bins represent the angle $\theta$ on one axis and offset from the centre, $S$ on the other. The sinogram corresponding to the activity distribution in figure $1.13 \mathrm{a}$ ) is shown in $1.13 \mathrm{~b}$ ). 


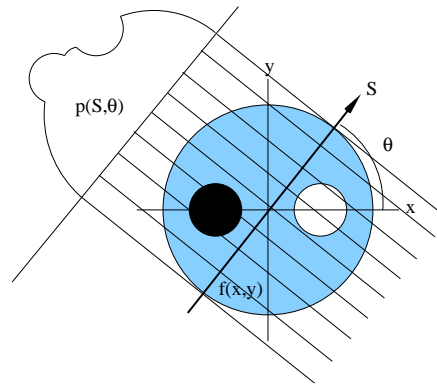

(a)

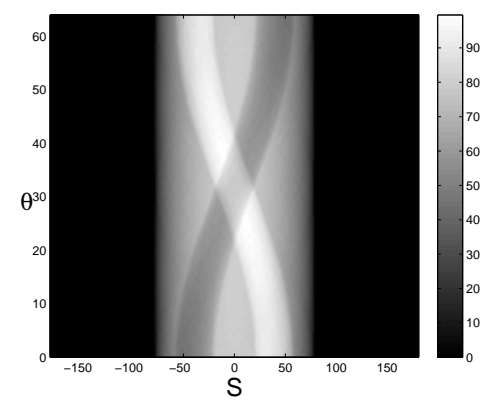

(b)

Figure 1.13: 2D PET data represented as projections. An activity distribution $f(x, y)$, consisting of an active disk with a hot, highactivity region (in black) and a cold, zero-activity region (in white) is represented in a). Also shown are parallel line integrals through $f(x, y)$ which are grouped into a projection $p(S, \theta)$. A sinogram of the distribution is shown in b).

\section{Backprojection}

The process of backprojection is the distribution of the counts in a detector pair into the image pixels that lie along the line joining the two detectors. The value given to each pixel is weighted by the length of the line that passes thought it. The backprojection of measured counts from all detector pairs is summed to form the backprojected image, which is an approximation of the original activity distribution $f(x, y)$. Since counts are distributed equally along the length of the line from which they originated, the backprojected image is a blurred representation of $f(x, y)$ which can be designated as $f^{\prime}(x, y)$. The operation can be expressed mathematically as the sum of sinogram elements from all $N$ projections $p\left(S, \theta_{n}\right)$ : 


$$
f^{\prime}(x, y)=\frac{1}{N} \sum_{n=1}^{N} p\left(S(x, y), \theta_{n}\right) .
$$

The projection variable $S$ is related to the image coordinates $(x, y)$ using the relation

$$
S(x, y)=x \cos \left(\theta_{n}\right)+y \sin \left(\theta_{n}\right) .
$$

The values of the variable $S$ in the sinogram will generally not coincide with the exact $(x, y)$ coordinates of image pixels and interpolation is necessary to calculate the sinogram contribution to each pixel.

\section{Filtered backprojection}

Filtered backprojection (FBP), as the name suggests, utilizes a method similar to backprojection but incorporates a high-pass filter into the projection data. The filter gives a larger weight to higher spatial frequencies that has the effect of reversing the blurring caused by backprojection. The mathematical operation is analogous to the equation for simple backprojection. It is expressed as

$$
f(x, y)=\frac{1}{N} \sum_{n=1}^{N} p^{*}\left(S, \theta_{n}\right) .
$$

To obtain $p^{*}(S, \theta)$, first a 1 -D Fourier transform of the original projection data $p(S, \theta)$ is taken with respect to the offset variable $S$ :

$$
P\left(\nu_{S}, \theta\right)=\mathscr{F}[p(S, \theta)]
$$

Then $P\left(\nu_{S}, \theta\right)$ is modified in spatial frequency domain, using a ramp filter:

$$
p^{*}(S, \theta)=\frac{1}{2 \pi} \mathscr{F}^{-1}\left[P\left(\nu_{S}, \theta\right) \times\left|\nu_{S}\right|\right] .
$$


This 2-D method is extended into 3-D reconstruction by means of the 3-D reprojection algorithm [42]. FBP is robust and computationally is relatively fast. However, it also has some limitations, which are discussed below.

\section{Limitations}

Analytical reconstruction approaches have a number of drawbacks. The method is based on ideal line integrals and does not model other aspects of the data collection process. These can include effects such as finite detector dimensions, inter-crystal scatter, acollinearity, patient motion, etc. Since statistical noise in the emission and detection processes is not modeled, it is present in the reconstructed images. Moreover, analytic reconstruction requires equally spaced sampling in the projection data [3], which is not always suitable for reconstructing data of variable resolution or data which has missing projections. The system presented in this work produces truncated, multi-resolution data and requires a more flexible reconstruction method. Statistic iterative methods provide the desired flexibility and have been used throughout this work.

\subsubsection{Iterative Reconstruction}

Unlike analytical reconstruction, which is based on the inverse transform of ideal line integrals, iterative techniques treat reconstruction as an optimization problem, which seeks the solution which best fits the measurements. These algorithms can be broadly classified as algebraic and statistical. The main advantage of algebraic iterative algorithms is that they can be very fast, while statistical iterative methods offer the possibility of directly modeling noise, making them more suitable for reconstructing noisy data [43]. Physical models for emission and detection can be included in iterative algorithms to better account for 
such effects during image reconstruction. Position-variant resolution and attenuation, for example, can be modeled in a straight-forward manner, which gives these algorithms considerable flexibility [43]. In addition, iterative methods are better at handling missing data resulting from truncation or inter-detector gaps. Finally, patient motion may be included into the acquisition model [44].

The iterative process uses a feedback loop to compare image estimates with the physical measurements and makes necessary adjustments for a closer fit. More specifically, an initial image estimate and a model of the data collection process are used to generate the projections that would result from that image estimate. The estimated projections are then compared to the measured projections and the image estimate updated accordingly. The updated image then serves as the input for the next iteration and the process is repeated, stopping when a predetermined condition is met [43].

Although more computationally demanding, iterative methods have replaced analytic ones in clinical practice, thanks to advances in computer technology.

\section{The system matrix}

In order to estimate the projections from an input distribution, a model of the emission and detection process of the system is required. In other words, the operator $\mathbf{A}$ from equation 1.5 must be estimated. The model must be able to determine the probability with which a positron, emitted at a certain position in the FOV, is detected as a coincidence event, in a certain pair of detectors. If the image space is discretized using the index $\mathrm{j}$ and the projection space using the index $i$, then the system model can be expressed as a matrix with elements $a_{i j}$, called the system matrix, which gives the probability with which an emission from pixel $\mathrm{j}$ is detected as measurement $\mathrm{i}$. Using the system matrix, forward projection is expressed as 


$$
\left\langle p_{i}\right\rangle=\sum_{j} a_{i j} f_{j}
$$

In this expression $\left\langle p_{i}\right\rangle$ is the expected, or mean value of projection $p_{i}$, given voxel values $f_{j}$. The imaging process itself is a specific realization of the projection operation in which noise is included. Similarly, an image estimate $f_{j}^{\prime}$ is obtained through backprojection, expressed as

$$
f_{j}^{\prime}=\sum_{i} a_{i j} p_{i}
$$

A system matrix consisting of a line integral model including attenuation correction and normalization results in a sparse matrix whose elements may be calculated at reconstruction time, that is on the fly. Including scatter in the model complicates the calculation and densely populates the matrix [45]. The system matrix can be expressed as a product of several matrices, each modeling a specific aspect of the imaging process [46]. These different factors may be calculated analytically or through simulation. Besides on-the-fly calculation, the system matrix may be pre-calculated and stored in memory.

\section{Statistical algorithms}

In general, PET data is noisy due to low count rates. A unique solution to the reconstruction problem cannot be obtained in such a case, so one that best fits the data is more suitable. Statistical algorithms consider the best solution to be the one that is most likely, given the measurements [43]. In other words, they look for a solution that maximizes the conditional probability $\operatorname{Pr}[\mathbf{f} \mid \mathbf{p}]$, the probability of achieving an image $\mathbf{f}$ given projection data $\mathbf{p}$. Using Bayes' theorem, this can be expressed in terms of the probability of measuring $\mathbf{p}$ given a distribution $\mathbf{f}$ : 


$$
\operatorname{Pr}[\mathbf{f} \mid \mathbf{p}]=\frac{\operatorname{Pr}[\mathbf{p} \mid \mathbf{f}] \operatorname{Pr}[\mathbf{f}]}{\operatorname{Pr}[\mathbf{p}]}
$$

When optimizing with respect to $\mathbf{f}, \mathbf{p}$ can be assumed to be constant and the problem is reduced to maximizing $\operatorname{Pr}[\mathbf{p} \mid \mathbf{f}] \operatorname{Pr}[\mathbf{f}]$. The factor $\operatorname{Pr}[\mathbf{p} \mid \mathbf{f}]$, called the likelihood, is a measure of how well the data agrees with the image. $\operatorname{Pr}[\mathbf{f}]$, called the prior, contains information about what is already known about the image. Reconstruction using an assumed prior is performed using maximum-a-posteriori (MAP) algorithms. If, on the other hand, we assume that nothing is know $a$ priori about the image, then $\operatorname{Pr}[\mathbf{f}]$ is also constant and the problem becomes maximizing the likelihood. Maximum-likelihood (ML) reconstruction looks for a solution that maximizes the likelihood. Since ML reconstruction will be the focus of our discussion, we assume a uniform prior from this point on.

Assuming that noise between detector locations is uncorrelated, the likelihood can be factorized into the contributions from the individual sinogram pixels $\mathrm{i}$ :

$$
\operatorname{Pr}[\mathbf{p} \mid \mathbf{f}]=\prod_{i} \operatorname{Pr}\left[p_{i} \mid \mathbf{f}\right]
$$

ML algorithms attempt to maximize this probability or, equivalently, its logarithm. The log-likelihood is given by

$$
l(f)=\sum_{i} \ln \operatorname{Pr}\left[p_{i} \mid \mathbf{f}\right]
$$

Poisson statistics, typically used to model radiation, are often employed as a model for PET measurements. According to this model, the probability of measuring a particular number of counts, c, given that the expected measurement is $\mathrm{m}$, is 


$$
\operatorname{Pr}[c \mid m]=\frac{e^{-m} m^{c}}{c !} .
$$

By substituting this into the factorized equation for likelihood (equation 1.14) we obtain:

$$
L(\mathbf{p} \mid \mathbf{f})=\operatorname{Pr}[\mathbf{p} \mid \mathbf{f}]=\prod_{i} \exp \left[-\sum_{j} a_{i j} f_{j}\right]\left(\sum_{j} a_{i j} f_{j}\right)^{p_{i}}\left(p_{i} !\right)^{-1}
$$

\section{Maximum Likelihood - Expectation Maximization}

The most widely used method for finding the ML solution uses the expectation maximization (EM) algorithm, resulting in maximum likelihoodexpectation maximization (ML-EM) [47, 48]. This method follows the iterative reconstruction process described above and, in addition, guarantees convergence. At the $k$ th iteration, projections are calculated using the projection operation (equation 1.11) with an estimated activity distribution $f_{j}^{k}$ and an appropriate system matrix $a_{i j}$. The estimate is then updated in a way that maximizes the likelihood. For EM, this involves calculating the ratio of the individual measured projections and those estimated using equation 1.11, backprojecting this ratio (equation 1.12), multiplying by the estimated distribution and dividing by sensitivity. The algorithm can be expressed as

$$
f_{j}^{k+1}=\frac{f_{j}^{k}}{S_{j}} \sum_{i} a_{i j} \frac{p_{i}}{\sum_{j} a_{i j} f_{j}^{k}} .
$$

An important feature in the above equation is the factor $S_{j}$ which accounts for system sensitivity. This is defined as the probability that an emission in $\mathrm{j}$ is detected by the scanner, and is calculated by backprojecting one count from every projection bin. It is expressed by the formula: 


$$
S_{j}=\sum_{i} a_{i j}
$$

\section{Limitations of ML-EM}

With every iteration of the ML-EM algorithm, the likelihood increases and the image estimate theoretically becomes closer to the true activity distribution [43]. However, in practice, an iteration number is reached at which optimal visual quality is achieved and beyond which the image becomes noisier. The high frequencies of the noise are amplified while the lower frequency information of the activity distribution becomes less visible. It is common practice to stop at a low number of iterations to limit noise. This practice, however, may reduce the accuracy of the reconstruction since the rate of convergence is dependent on activity concentration [45] and may not be the same in all points of the reconstructed image. This problem can be avoided by using a higher number of iterations and smoothing the resulting image [49] or by using penalized ML-EM [50], equivalent to MAP. Another limitation of the algorithm is computation time. Each iteration involves a forward and back projection and therefore takes about twice the computational time as FBP. However, there are methods for significantly accelerating reconstruction using variations of ML-EM, most notably the widely used ordered subset expectation maximization (OSEM) algorithm [51].

\section{List-Mode ML-EM}

For certain applications, as when using continuous detectors, where the spatial discretization of the measurements leads to loss of information, it is more appropriate to use a list-mode version of the ML-EM algorithm [52]. Using this method, the main summation runs through 
the $\mathrm{N}$ events in the list-mode data $(l=1, \cdots, N)$. The algorithm is given by:

$$
f_{j}^{k+1}=\frac{f_{j}^{k}}{S_{j}} \sum_{l=1}^{N} a_{i_{l} j} \frac{1}{\sum_{b=1}^{J} a_{i_{l} b} f_{b}^{k}} .
$$

Here, $b=1, \cdots, J$ is the pixel index in the projection operation. The system matrix $a_{i_{l} j}$ is the probability that a detected emission from pixel $j$ is detected in the $i^{\text {th }}$ detector-pair, corresponding to event $l$. The list-mode ML-EM algorithm is used for image reconstruction throughout this work.

\subsection{Summary}

In this chapter a review of the fundamental concepts of PET imaging has been presented. The basic working principles of this imaging technique have been described: the concept of the radiopharmaceutical and how it is able to concentrate in certain areas of the body which are of clinical interest, and the fundamental physical processes that transmit this information to the outside world. The limitations of PET imaging have also been explained. It has been shown how resolution is degraded by certain physical effects. A review of the basic detector technology used in PET scanners has been presented as well as the idea of using $\mathrm{Si}$ as an unconventional detector material. An explanation of the different coincidence events has been included. Finally, a summary of the image reconstruction methods used in PET has been presented with a focus on the ML-EM algorithm. In the above discussion, a conceptual basis has been laid for the detailed examination of a the high-resolution PET probe system presented in this work. 


\section{References}

[1] Smith, W.L., Farrell, T.A.: Nuclear imaging. In: Radiology 101: The Basics \& Fundamentals of Imaging. Lippincott Williams \& Wilkins (2013) 337-357

[2] Muzi, M., O’Sullivan, F., Mankoff, D.A., Doot, R.K., Pierce, L.A., Kurland, B.F., Linden, H.M., Kinahan, P.E.: Quantitative assessment of dynamic PET imaging data in cancer imaging. Magn Reson Imaging 30(9) (2012) 1203 - 15

[3] Simon R. Cherry and Magnus Dahlbom. In: PET: Physics, Instrumentation, and Scanners. Springer, New York (2006)

[4] Harpen, M.D.: Positronium: Review of symmetry, conserved quantities and decay for the radiological physisist. Med. Phys. 31(1) (Jan 2004) 57-61

[5] Czarnecki, A., Karshenboim, S.G.: Decays of positronium. In: Proceedings of the International Workshop on High Energy Physics and Quantum Field Theory (QFTHEP). (May 1999) 538544

[6] Karshenboim, S.G.: Precision study of positronium: Testing bound state QED theory. Int. J. Mod. Phys. A 19 (2003) 38793896

[7] Levin, C.S., Hoffman, E.J.: Calculation of positron range and its effect on the fundamental limit of positron emission tomography system spatial resolution. Phys. Med. Biol. 44 (1999) 781-799

[8] Tai, Y.C., Wu, H., Pal, D., O'Sullivan, J.A.: Virtual-pinhole PET. J. Nucl. Med. 49 (2008) 471 
[9] Simon R. Cherry and James A. Sorenson and Michael E. Phelps. In: Physics in Nuclear Medicine. Saunders, Philadelphia (2003)

[10] Knoll, G.F.: Radiation Detection and Measurement, Third Edition. John Wiley and Sons, Inc. (2000)

[11] Surti, S., Karp, J.S., Popescu, L.M., Daube-Witherspoon, M.E., Werner, M.: Investigation of time-of-flight benefit for fully 3-D PET. IEEE Trans. Med. Imag. 25 (2006) 259-269

[12] Lewellen, T.K.: Recent developments in PET detector technology. Phys. Med. Biol. 53 (2008) R287-R317

[13] Frach, T., Ruetten, W., Fiedler, K., Maehlum, G., Solf, T., Thon, A.: Assessment of photodiodes as a light detector for PET scanners. In: Nuclear Science Symposium Conference Record, 2004 IEEE. Volume 7. (Oct 2004) 4177-4181

[14] Herbert, D.J., Moehrs, S., D’Ascenzo, N., Belcari, N., DelGuerra, A., Morsani, F., Saveliev, V.: The silicon photomultiplier for application to high-resolution positron emission tomography. Nucl. Instrum. Methods A 573 (2007) 84-87

[15] McElroy, D.P., Saveliev, V., Reznik, A., Rowlands, J.A.: Evaluation of silicon photomultipliers: A promising new detector for MR compatible PET. Nucl. Instrum. Methods A 571 (2007) 106-109

[16] Costa, E., Massaro, E., Piro, L.: A BGO-CsI(TI) phoswich: a new detector for x-and y-ray astronomy. Nucl. Instrum. Methods A 243 (1986) 572577

[17] Braem, A., Chesi, E., Ciocia, F., Leo, R.D., Joram, C., Lagamba, L., Nappi, E., S'eguinot, J., Vilardi, I., Weilhammer, P.: Scintil- 
lator studies for the HPD-PET concept. Nucl. Instrum. Methods A 571 (2007) 419-24

[18] Rafecas, M., Boning, G., Pichler, B., Lorenz, E., Schwaiger, M., Ziegler, S.: A Monte Carlo study of high-resolution PET with granulated dual-layer detectors. Nuclear Science, IEEE Transactions on 48(4) (Aug 2001) 1490-1495

[19] Nishikido, F., Inadama, N., Yoshida, E., Murayama, H., Yamaya, T.: Four-layer DOI PET detectors using a multi-pixel photon counter array and the light sharing method. Nucl. Instrum. Methods A 729 (2013) 755-761

[20] Shao, Y., Li, H., Gao, K.: Initial experimental studies of using solid-state photomultiplier for PET applications. Nucl. Instrum. Methods A 580 (2007) 944-950

[21] Lewellen, T., Janes, M., Miyaoka, R.: Dmice-a depth-ofinteraction detector design for pet scanners. In: Nuclear Science Symposium Conference Record, 2004 IEEE. Volume 4. (Oct 2004) 2388-2392 Vol. 4

[22] Schäfers, K.P., Reader, A.J., Kriens, M., Knoess, C., Schober, O., Schäfers, M.: Performance evaluation of the 32-module quadHIDAC small-animal PET scanner. J. Nucl. Med. 46 (2007) 9961004

[23] Tsyganov, E., Anderson, J., Arbique, G., Constantinescu, A., Jennewein, M., Kulkarni, P., Mason, R., McColl, R., Oz, O., Parkey, R., Richer, E., Rosch, F., Seliounine, S., Slavine, N., Srivastava, S., Thorpe, P., Zinchenko, A., Antich, P.: UTSW small animal positron emission imager. Nuclear Science, IEEE Transactions on 53(5) (Oct 2006) 2591-2600 
[24] Blanco, A., Carolino, N., Ferreira, N., Correia, C.M.B.A., Macedo, M.P., Fazendeiro, L., Marques, M., Marques, R., Gil, C., Fonte, P.: RPC-PET: a new very high resolution PET technology. In: Nuclear Science Symposium Conference Record, 2004 IEEE. Volume 4. (Oct 2004) 2356-2360 Vol. 4

[25] Park, S., Rogers, W., Huh, S., Kagan, H., Honscheid, K., Burdette, D., Chesi, E., Lacasta, C., Llosá, G., Mikuž, M., Studen, A., Weilhammer, P., Clinthorne, N.: A prototype of very highresolution small animal PET scanner using silicon pad detectors. Nucl. Instrum. Methods A 570 (2007b) 543

[26] Clinthorne, N., et al.: Very high resolution animal PET. J. Nucl. Med. 41 (2000) 20

[27] Cooper, R.J., Boston, A.J., Boston, H.C., Cresswell, J.R., Grint, A.N., Harkness, L.J., Nolan, P.J., Oxley, D.C., Scraggs, D.P., Lazarus, I., Simpson, J., Dobson, J.: Charge collection performance of a segmented planar high-purity germanium detector. Nucl. Instrum. Methods A 595 (2008) 401-409

[28] Shiga, T., Morimoto, Y., Kubo, N., Katoh, N., Katoh, C., Takeuchi, W., Usui, R., Hirata, K., Kojima, S., Umegaki, K., Shirato, H., Tamaki, N.: A new PET scanner with semiconductor detectors enables better identification of intratumoral inhomogeneity. J. Nucl. Med. 50 (2009) 148-155

[29] Gu, Y., Matteson, J.L., Skelton, R.T., Deal, A.C., Stephan, E.A., Duttweiler, F., Gasaway, T.M., Levin, C.S.: Study of a highresolution, 3D positioning cadmium zinc telluride detector for PET. Phys. Med. Biol. 56 (2011) 1563-1584 
[30] Meier, D., Czermak, A., Jalocha, P., Sowicki, B., Kowal, M., Dulinski, W., Maehlum, G., Nygard, E., Yoshioka, K., Fuster, J., Lacasta, C., Mikuž, M., Roe, S., Weilhammer, P., Hua, C.H., Park, S.J., Wildermann, S., Zhang, L., Clinthorne, N., Rogers, W.: Silicon detector for a compton camera in nuclear medical imaging. Nuclear Science, IEEE Transactions on 49(3) (Jun 2002) 812-816

[31] Brasse, D., Kinahan, P., Lartizien, C., Comtat, C., Casey, M., Michel, C.: Correction methods for random coincidences in fully 3D whole-body PET: impact on data and image quality. J Nucl Med. 46 (2005) 859-67

[32] Oliver, J.F., Rafecas, M.: Improving the singles rate method for modeling accidental coincidences in high-resolution PET. Phys. Med. Biol 55 (2010) 6951-6971

[33] Stearns, C.: Scatter correction method for 3D PET using 2D fitted gaussian functions. J Nucl Med. 36 (1995) 105P

[34] Cherry, S., Huang, S.C.: Effects of scatter on model parameter estimates in 3D PET studies of the human brain. IEEE Trans. Nucl. Sci. 42(4) (1995) 1174-1179

[35] Ollinger, J.: Model-based scatter correction for fully $3 \mathrm{~d}$ pet. Phys. Med. Biol. 41 (1996) 153-176

[36] Watson, C., Newport, D., Casey, M.: A single-scatter simulation technique for scatter correction in 3D PET. Three-Dim. Imag. Recon. Rad. Nucl. Med. 4 (1996) 255-268

[37] Komarov, S.A., Wu, H., Keesing, D.B., O’Sullivan, J.A., Tai, Y.C.: Compton scattering in clinical PET/CT with high resolu- 
tion half ring PET insert device. IEEE Trans. Nucl. Sci. 57(3) (1996) 1045-1051

[38] Levin, C., Dahlbom, M., Hoffman, E.: A monte carlo correction for the effect of compton scattering in 3-d pet brain imaging. Nuclear Science, IEEE Transactions on 42(4) (Aug 1995) 11811185

[39] Derenzo, S.E.: Recent developments in positron emission tomography (PET) instrumentation. SPIE - Physics and Engineering of Computerized Multidimensional Imaging and Processing 671 (1986) 232-243

[40] Moses, W.W., Derenzo, S.E.: Empirical observation of resolution degradation in positron emission tomographs utilizing block detectors. J. Nucl. Med. 34 (1993) 101P

[41] Tomic, N., Thompson, C., Casey, M.: Investigation of the "Block Effect" on spatial resolution in PET detectors. Nuclear Science, IEEE Transactions on 52(3) (2005) 599-605

[42] Kinahan, P., Rogers, J.: Analytic 3d image reconstruction using all detected events. Nuclear Science, IEEE Transactions on 36(1) (Feb 1989) 964-968

[43] Brian F. Hutton and Johan Nuyts and Habib Zaidi: Iterative Reconstruction Methods. In: Quantitative Analysis in Nuclear Medicine Imaging. Springer, New York (2006) 107-140

[44] Blume, M., Navab, N., Rafecas, M.: Joint image and motion reconstruction for PET using a B-spline motion model. Phys. Med. Biol. 57 (2012) 8249-8270 
[45] Michel Defrise and Paul E. Kinahan and Christian J. Michel: Image Reconstruction Algorithms in PET. In: Positron Emission Tomography. Springer-Verlag, London (2005) 63-91

[46] Qi, J., Leahy, R.M., Cherry, S.R., Chatziioannou, A., Farquhar, T.H.: High-resolution 3D bayesian image reconstruction using the microPET small-animal scanner. Phys. Med. Biol 43 (1998) 10011013

[47] Shepp, L.A., Vardi, Y.: Maximum likelihood reconstruction for emission tomography. Medical Imaging, IEEE Transactions on $\mathbf{1}(2)$ (Oct 1982) 113-122

[48] Lange, K., Carson, R.: EM reconstruction algorithms for emission and transmission tomography. J Comput Assist Tomogr 8(2) (1984) $306-16$

[49] Nuyts, J.: On estimating the variance of smoothed mlem images. Nuclear Science, IEEE Transactions on 49(3) (Jun 2002) 714-721

[50] Nuyts, J., Bequé, D., Dupont, P., Mortelmans, L.: A concave prior penalizing relative differences for maximum-a-posteriori reconstruction in emission tomography. Nuclear Science, IEEE Transactions on 49(1) (Feb 2002) 56-60

[51] Hudson, H.M., Larkin, R.S.: Accelerated image reconstruction using ordered subsets of projection data. IEEE Trans. Med. Imag. 13 (1994) 601-609

[52] Barrett, H., White, T., Parra, L.: List-mode likelihood. J. Opt. Soc. Am. A 14 (1997) 2914 


\section{Chapter 2}

\section{THE PET PROBE CONCEPT}

\section{$2.1 \quad$ Introduction}

One of the most important challenges in PET imaging is spatial resolution. Modern clinical scanners typically achieve values of $5 \mathrm{~mm}$ FWHM [1] for reconstructed image resolution, compared to the submillimeter values achievable by clinical CT [2] and MRI [3]. The factors limiting image resolution in PET are listed in chapter 1 (section 1.4.2) and their relative contribution to image resolution, at the centre of a scanner's FOV, was quantified by an empirical formula (equation 1.4, page 35). It follows from this formula that there are three approaches to increasing resolution in PET: reducing the effects of acollinearity, detector size and block effect. Source size, of course, is independent of scanner geometry. These approaches are employed, in varying combinations, in several PET-insert geometries including the probe system. 


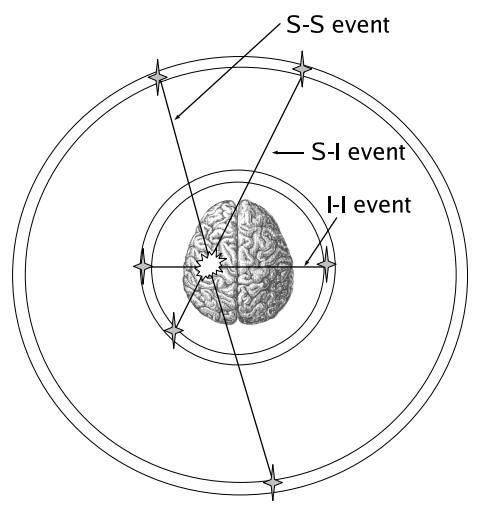

Figure 2.1: Schematic diagram of a ring insert geometry where the insert detectors form a full ring around the object being imaged. The three types of coincidence events are shown: scanner to scanner (S-S), scanner to insert (I-S) and insert to insert (I-I).

The PET-insert concept involves placing additional detectors in the FOV of a PET scanner. The inserted detectors are connected in coincidence with one another, as well as with the PET ring itself and are therefore able to simultaneously detect annihilation photon pairs. In the most general case there are three types of coincidence events: those between two insert detectors (insert to insert, I-I events), those between an insert detector and a detector in the PET scanner (insert to scanner, I-S events) and conventional PET coincidences between two detectors in the PET scanner (scanner to scanner, S-S events). The three types of coincidence events are shown schematically in figure 2.1 for a specific geometry where insert detectors form a ring around the object.

Insert systems can be broadly classified into two categories: ring and probe devices. The ring insert geometry involves multiple detectors placed around the object being imaged, in the form of a ring 


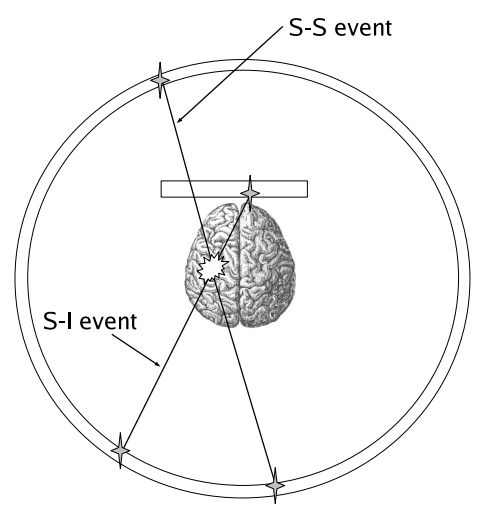

Figure 2.2: Schematic diagram of a probe geometry with the highresolution detector in close proximity to the object being imaged. Included are the two types of coincidence events: S-S and I-S.

(as shown in figure 2.1) or partial ring. The probe insert consists of a single detector (or stack) on one side of the object, as shown schematically in figure 2.2.

This chapter explains the working principles of the PET-insert technique and its two variations. It also provides an overview of the various implementations of these techniques, as developed by several research groups.

\subsection{Effect on resolution}

Equation 1.4 applies to the resolution of a cylindrical scanner at the centre of its FOV. For an insert geometry certain modifications must be made. Those related to photon acollinearity and detector size have been elaborated by Tai et al [4]. The contribution due to block effect depends on the type of detector used and can be greatly reduced using detectors employing one-to-one coupling, made possible by the advances in photodetector technology described in section 1.3.1. This section explores the factors which limit PET spatial resolution, in 
the context of the PET insert concept. The individual and combined effects of photon acollinearity and detector size on resolution of the three event types are quantified. The block effect is briefly discussed.

\subsubsection{Photon acollinearity}

At the centre of a cylindrical scanner $R_{a c l n}$, the FWHM of the blurring function due to annihilation-photon acollinearity, is expressed as

$$
R_{a c l n}=0.0022 \times D,
$$

where $D$ is the diameter of the scanner. However, for other positions along the LOR, a more general expression is

$$
R_{a c l n}=0.0088 \times \frac{d_{1} \times d_{2}}{d_{1}+d_{2}},
$$

where $d_{1}$ and $d_{2}$ are the distances to the two detectors and the factor 0.0088 is due to the angular distribution of the photon acollinearity, which is approximately $0.5^{\circ}$ or $0.0088 \mathrm{rad}$ FWHM [4]. In the centre of the FOV, where $d_{1}=d_{2}=D / 2$, equation 2.2 reduces to equation 2.1. It is clear from equation 2.2 that if one or both of the detectors are brought in closer to the object, $R_{a c l n}$ is reduced and therefore higher resolution is achieved.

To demonstrate this effect, the acollinearity component of spatial resolution blurring for several detector pair geometries is plotted in figure 2.3. $R_{a c l n}$ is plotted for detectors positioned at $x_{1}$ and $x_{2}$, as a function of position along the LOR. The case of a clinical PET scanner is represented by two detectors separated by $1000 \mathrm{~mm}$, approximately the diameter of a typical scanner. Figure 2.3 a) demonstrates the effect of bringing both detectors closer to each other, at several smaller diameters. The plots corresponds to S-S events when $x_{1}=0 \mathrm{~mm}$ and $x_{2}=1000 \mathrm{~mm}$ and I-I events for other positions. Figure $2.3 \mathrm{~b}$ ) demonstrates the effect of placing one of the detectors closer to the 


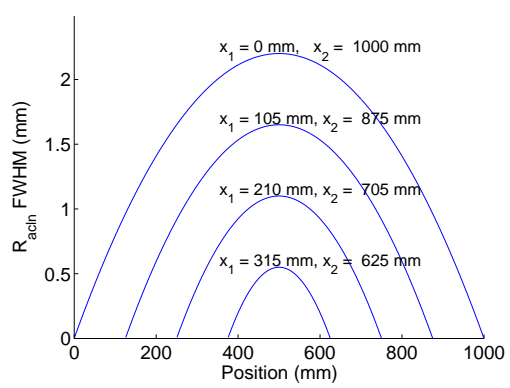

(a)

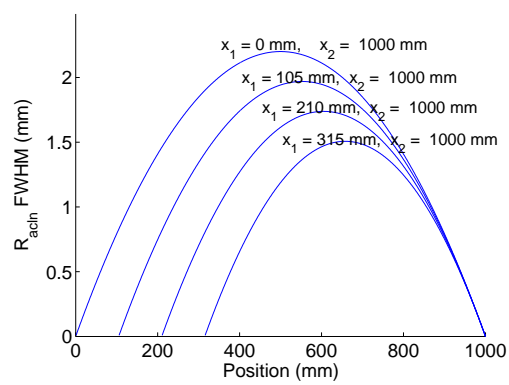

(b)

Figure 2.3: Resolution loss due to photon acollinearity as a function of position for a) I-I events and b) I-S events. The case where $\mathrm{x}_{1}$ $=0 \mathrm{~mm}$ and $\mathrm{x}_{2}=1000 \mathrm{~mm}$ corresponds to the $\mathrm{S}-\mathrm{S}$ events and is included in both plots for reference. Resolution is expressed in terms of the FWHM of the blurring function. Given a constant insert offset position, improvement is greater in the case of an I-I event.

centre at several offset positions. These plots correspond to I-S events with S-S events $\left(x_{1}=0 \mathrm{~mm}\right.$ and $x_{2}=1000 \mathrm{~mm}$ for reference. It can be seen that in both cases (I-I and I-S) resolution is improved. The PET insert geometries exploit this effect by placing detectors close to the object.

\subsubsection{Detector size}

The effect of detector size on the intrinsic resolution of a cylindrical PET scanner was described in section 1.2.2. For two identical, faceto-face detectors (as in the central LOR), the coincidence response function $(\mathrm{CRF})$ is symmetric about the midplane, with a base width equal to the detector width $w$. At the midplane the CRF is triangular, with FWHM $=w / 2$ (figure 1.3, page 17). The value $w / 2$ is used in equation 1.4 for resolution loss due to detector size. If the two detec- 
tors involved in the coincidence measurement are of different widths, $w_{1}$ and $w_{2}$, the variation of the CRF becomes asymmetric along the $\mathrm{LOR}$, with respect to its centre (figure $2.4 \mathrm{~b}$ ). The CRF still becomes triangular in shape but now this occurs at a point closer to the detector with smaller width. As in the case of identical detectors, the CRF becomes rectangular at the two detector surfaces, but now with widths $w_{1}$ and $w_{2}$. It becomes trapezoidal at intermediate locations, as with identical detectors, but now its base and plateau widths are modified due to the difference in detector widths. Figure 2.4 illustrates the differences in the CRF for a pair of identical detectors and for a pair where one of the detectors is smaller.

For two detectors of widths $w_{1}$ and $w_{2}$, separated by a distance $D$, the equation for the FWHM of the spatial resolution blurring function due to detector size is:

$$
R_{\text {det }}=\frac{(D-x) \cdot w_{1}+x \cdot w_{2}+\left|(D-x) \cdot w_{1}-x \cdot w_{2}\right|}{2 \cdot D}
$$

where $x$ is the distance from the detector of width $w_{1}$ [4]. Rewriting in terms of the variables $d_{1}$ and $d_{2}$, as in equation 2.2 , the expression becomes:

$$
R_{d e t}=\frac{d_{2} \cdot w_{1}+d_{1} \cdot w_{2}+\left|d_{2} \cdot w_{1}-d_{1} \cdot w_{2}\right|}{2 \cdot\left(d_{1}+d_{2}\right)} .
$$

Intrinsic resolution due to detector size, $R_{\text {det }}$, is plotted in figure 2.5 for several detector pair geometries. The FWHM is plotted for face-to-face detectors, positioned at $x_{1}$ and $x_{2}$, as a function of position along their LOR. The detector pairs correspond to S-S, I-I and I-S events. The scanner detectors are $4 \mathrm{~mm}$ wide, a typical crystal width in a modern clinical scanner, and separated by $1000 \mathrm{~mm}$. The highresolution insert detector width was chosen to be $1 \mathrm{~mm}$. The detector 


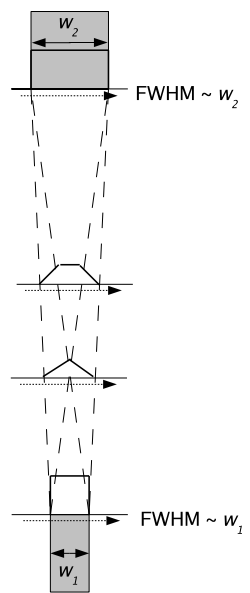

Figure 2.4: The CRF for a pair of detectors as it varies along the length of the LOR. If the detectors are identical (as shown in figure 1.3, the CRF is triangular at the midpoint and becomes trapezoidal towards the two detectors in a symmetric fashion. Intrinsic resolution is highest where the CRF is triangular and degrades as it spreads to become trapezoidal. If one of the detectors is narrower (as shown here), then the symmetry is broken and the CRF becomes triangular and narrower at a point closer to the small detector.

sizes were chosen to match those used in simulation studies of the probe system, presented in coming chapters.

Resolution for S-S and I-I events, with the insert detectors at several offsets, is shown in figure 2.5 a). The plots demonstrate how decreasing the size of both detectors, from $4 \mathrm{~mm}$ to $1 \mathrm{~mm}$, increases the intrinsic resolution, but that moving them to smaller radii reduces the improvement at a given point along the LOR. Figure $2.5 \mathrm{~b}$ ) shows resolution of I-S events with the insert at several offset positions and includes S-S resolution for reference. Figure $2.5 \mathrm{~b}$ ) demonstrates that decreasing the size of just one of the detectors also increases spa- 


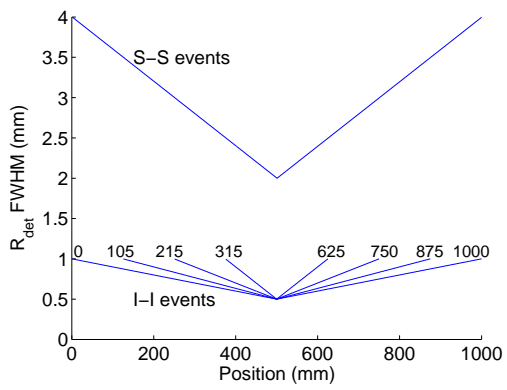

(a)

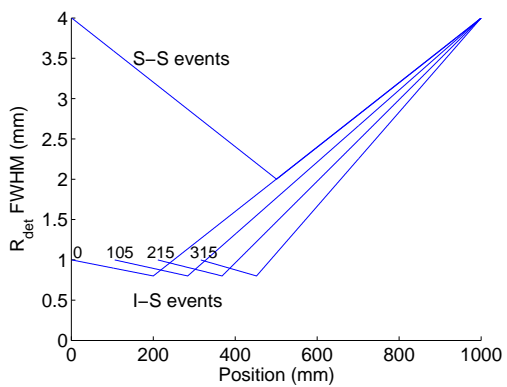

(b)

Figure 2.5: Resolution loss due to detector size as a function of position for a) I-I events and b) I-S events, with S-S events plotted for reference. The scanner and insert detector widths are $4 \mathrm{~mm}$ and $1 \mathrm{~mm}$ respectively. Both insert detectors are stepped in by equal amounts. Resolution is expressed in terms of the FWHM of the blurring function.

tial resolution. The degree of improvement at a particular location, however, is dependent on the offset of the insert detector relative to the centre of the FOV. The point of highest intrinsic resolution is located at some distance away from the insert detector. In figure 2.5 b) this distance ranges from about $200 \mathrm{~mm}$ and decreases as the detector is brought toward the centre. The point of highest resolution corresponds to the location along the LOR where the CRF becomes triangular.

Most state-of-the-art whole-body PET scanners achieve high spatial resolution by using crystal sizes between $4 \times 4 \mathrm{~mm}^{2}$ and $6 \times 6 \mathrm{~mm}^{2}$ in face area [1]. Further crystal segmentation would result in an increased complexity in detector readout, or alternatively a reduced FOV if the number of detectors were maintained. Reduction of crystal size also leads to an increase in inter-crystal scatter and events that are associated with the incorrect LOR. The choice of crystal size results in an intrinsic resolution-sensitivity trade-off. 


\subsubsection{Acollinearity and Detector Size Combined}

A more accurate representation of resolution variation across the FOV for the above geometries is obtained by looking at the combined effect of photon acollinearity and detector size, ignoring, for simplicity, the factor of 1.25 due to reconstruction. The resolution blurring due to the combination of the two effects is calculated by adding their individual components in quadrature:

$$
R_{c o m b}=\sqrt{R_{a c n l}^{2}+R_{d e t}^{2}} .
$$

Plots of $R_{\text {comb }}$, analogous to those in figures 2.3 and 2.5, are shown in figure 2.6. It can be seen from figure 2.6 a) that the resolution of I-I events is higher than that of the S-S events at all detector offsets and for all points along the LOR. It is also evident that resolution increases as the insert detectors are brought closer to the centre of the FOV. It is interesting to note that when both detectors are $4 \mathrm{~mm}$ wide (S-S event) the effect of detector size is the dominant contribution to resolution variation along the LOR, while with $1 \mathrm{~mm}$ detectors (I-I event) the effect of acollinearity dominates. An exception to this observation is the case where the insert detectors are at their closest to each other, at $\mathrm{x}_{1}=315 \mathrm{~mm}$ and $\mathrm{x}_{2}=625 \mathrm{~mm}, 310 \mathrm{~mm}$ apart. Here, detector size is once again the dominant component. From figure $2.6 \mathrm{~b}$ ) we observe that the resolution of I-S events is higher than that of the S-S events at all offsets and for all points along the LOR. A notable difference is when the $1 \mathrm{~mm}$ detector is located at the PET ring's radius. Here $R_{\text {comb }}$ for the I-S and S-S events are identical on the side of the LOR closer to the large detector. It was observed that resolution increases as the insert detector is brought closer to the centre of the FOV. The sharp dip in $R_{\text {det }}$ close to the insert detector (figure 2.5) is much less pronounced with the addition of acollinearity. $R_{\text {comb }}$ dips only slightly and rises back at about $10 \mathrm{~mm}$ from the detector surface. 


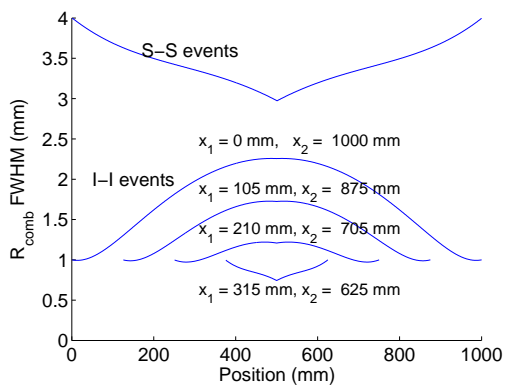

(a)

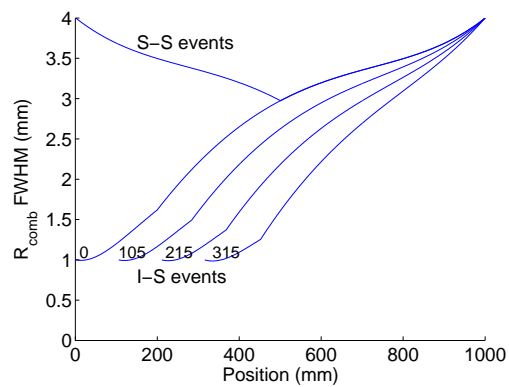

(b)

Figure 2.6: Resolution loss due to the combined effects of photon acollinearity and detector size as a function of position for a) I-I events and b) I-S events, with S-S events plotted for reference. Resolution is expressed in terms of the FWHM of the blurring function.

The resolution near the insert detector for a I-S LOR can be further explored by considering different pixel sizes for the insert detector. Figure 2.7 shows plots of resolution for an insert detector of varying size, placed at the scanner radius. Sizes ranging from $0.5 \mathrm{~mm}$ to 4 $\mathrm{mm}$ are used. Intrinsic resolution $R_{\text {det }}$ is plotted in figure 2.7 a) while $R_{\text {comb }}$ is plotted in b). It can be seen in a) that a reduction in insert detector size only produces resolution gains on one side of the point of lowest $R_{d e t}$, closer to the insert. The same is true when the effect of acollinearity is included, as shown in b). Although the behavior of the resolution $R_{\text {comb }}$ is different from $R_{\text {det }}$, the region affected by detector size reduction is the same.

\subsubsection{Block effect}

The block design of a typical scintillation detector in a PET scanner was described in section 1.3.1. The degradation to resolution inherent in this design is termed the block effect (explained in section 1.4.2). 


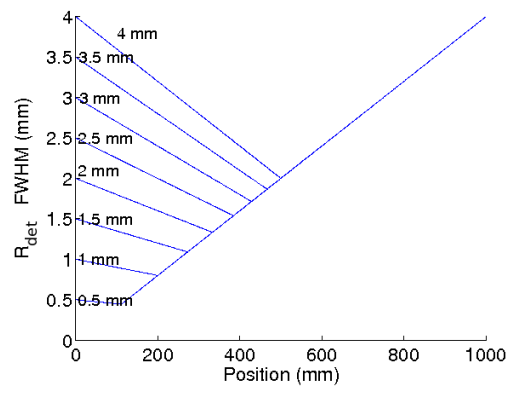

(a)

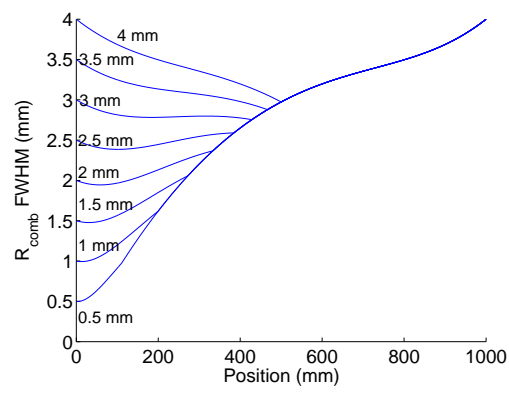

(b)

Figure 2.7: The effect of insert detector size on resolution for I-S events. For a pair of detectors separated by $1000 \mathrm{~mm}$, the plots demonstrate the effect of varying the size of one of the detectors on resolution. Plotted are a) the intrinsic resolution and b) the resolution due to the combined effects of photon acollinearity and detector size, as a function of position. Resolution is expressed in terms of the FWHM of the blurring function.

The degradation is caused by event mispositioning in the scintillation crystals due to the combined effect of several phenomena which occur inside a block detector [5]. One way of overcoming the problem of the block effect is to use a detector which does not follow the block design, for instance coupling every crystal individually to a photodetector. Advances in photodetector technology have allowed for the possibility of producing scintillator detectors with one-to-one coupling, as discussed in section 1.3.1. Although widely used in preclinical geometries, due to the large number of readout channels and the high cost of the detector, very few clinical PET scanners employ these devices ${ }^{1}$. For insert devices, which use a smaller number of detectors, this application is more realistic. Another possibility for

\footnotetext{
${ }^{1}$ The Philips Vereos PET/CT uses digital SiPMs to achieve one-to-one coupling
} 
individual pixel readout that can be applied in the context of insert geometries is the use of semiconductor detectors. These devices rely on the direct detection of gamma photons by a highly pixelated detector, in many cases with no signal multiplexing. Their application to PET imaging has been the subject of extensive investigation $[6,7,8]$.

\subsection{The Ring Insert Geometry}

The ring insert geometry consists of high-resolution detectors inside the FOV of a clinical PET scanner. They are placed around the object being imaged so as to detect coincidence events between each other as well as with the detectors of the clinical scanner. This definition includes geometries ranging from a pair of opposing insert detectors to a full ring, surrounding the object. Since all detectors are connected in coincidence, three types of events are possible in this configuration: S-S, I-S and I-I, as were defined in figure 2.1. The three event types can be reconstructed separately or combined during reconstruction to create a single image [9], with higher resolution than an image reconstructed using S-S events only.

\subsubsection{Working Principles}

By positioning highly-segmented detectors close to the object being imaged, high-resolution information can be obtained. As explained in section 2.2, this is because the effect of photon acollinearity is diminished and intrinsic resolution due to detector size is increased. Placing highly-segmented detectors at a small radius following a conventional PET scanner geometry is one method of realizing this goal. In addition, a sensitivity increase can be achieved by placing the smaller ring in the FOV of a clinical PET scanner, following the PET ring insert geometry. In such a system, S-S events are combined with high- 
resolution I-S and I-I events to create a single image. Experimental prototypes and simulation studies have demonstrated, to varying degrees, the increased spatial resolution of systems utilizing the ring insert concept (described below).

\subsubsection{Existing systems}

A small-animal PET imaging system which utilizes the ring insert geometry has been proposed using Si detectors [8]. An outer ring of $\mathrm{BGO}$ detectors provides high sensitivity while an inner ring of $\mathrm{Si}$ detectors collects high-resolution information. Simulation studies [10] and an experimental prototype [11] have demonstrated the ability of the system to achieve sub-millimeter resolution. An extension of the work using a human-scale scanner with BGO detectors and an inner Si-detector ring has also shown promising results [9]. The larger dimensions of the second prototype indicate the applicability to human subjects. Other ring insert prototypes, based on a commercial small-animal scanner and high-resolution LSO scintillator detectors, have been studied and demonstrate a marked improvement to image resolution as compared to the original system. These include a setup with a single insert detector rotated through $180^{\circ}$ [12] and a full-ring insert geometry [13]. Similar geometries, using LSO detectors in a ring insert, have been studied by the same group in application to whole body clinical scanners. Simulation studies have demonstrated the possibility of improved spatial resolution in this context [14]. A prototype system, based on a Siemens Biograph-40 clinical PET-CT scanner and a half-ring insert composed of high-resolution LSO detectors has been constructed [15] and shown to benefit image resolution and contrast $[15,16]$. The advantage of the half-ring insert is that while collecting all three types of events (S-S, I-S and I-I) it adds a certain degree of flexibility to patient positioning. Flexibility is further augmented when considering the probe-insert geometry. 


\subsection{The Probe Geometry}

An alternative to the ring insert is the probe. In a probe geometry, a single high-resolution detector, or a detector stack, is operated in coincidence with a PET scanner, providing increased spatial resolution and sensitivity in a region close to the detector surface. Such devices have been investigated by various groups $[17,18]$. The probe geometry offers several advantages over the ring insert, which will be described below.

\subsubsection{Working Principles}

In addition to regular S-S events, a probe system also collects highresolution I-S events (shown schematically in figure 2.2 ), which provide higher spatial resolution and increased sensitivity in the region close to the probe. As has been demonstrated, the resolution information contained in the I-S data depends strongly on the positioning of the probe and the size of its detectors. Furthermore, the proportion of I-S events in the data determines the degree with which the highresolution data serves to enhance the performance of the system.

\subsubsection{Existing systems}

A probe geometry was utilized in simulation studies of an internal PET probe for prostate imaging [17], an inter-operative imaging probe [19] and the less invasive zoom-in PET small-animal system [18]. These probes could be positioned in close proximity to the prostate, tissue accessible through surgery and tumors near the skin surface, respectively. By means of simulation studies Zhou \& Qi have shown that the spatial resolution of a small-animal PET scanner can be increased by combining the scanner with a high-resolution, depth-of-interaction (DOI) LSO detector probe. Their studies have also shown that the 
zoom-in PET system offers higher lesion detectability [18]. The study of an experimental prototype of this system, which has also shown an improvement in spatial resolution [20], is a further demonstration of the potential of such a device. Another PET probe that is currently under development for use in close proximity to the object, employs high-resolution Si detectors [21] for use in coincidence with a clinical scanner. The work presented here is an elaboration of the Si probe system.

\subsubsection{Advantages and disadvantages}

A probe is more straightforward to adapt to an existing system and less costly as it makes use of only one high-resolution detector, although several layers can be used to increase sensitivity. Flexibility in probe positioning allows for the placement of the high-resolution detector closer to the region of interest (ROI), taking full advantage of the available resolution. The geometry also allows for task-specific positioning of the probe with respect to the subject being imaged, thus allowing the possibility of adaptive imaging [22]. While a probe insert provides many advantages, there are also some disadvantages associated with this type of imaging system. These include a narrow range of projection angles in the probe data and variable resolution. These characteristics of the probe data present a challenge for image reconstruction and in determination of the optimal position for probe placement. A further difficulty, which the probe shares in common with the ring insert, arises due to the attenuation and scatter produced by the additional detectors in the FOV of the conventional scanner. 


\subsection{Clinical applications of the PET probe}

The clinical application of the PET probe system would be to imaging problems where higher resolution is desired in a localized ROI. This can include situations where a lesion is located close to the body surface, within a predetermined range of increased resolution provided by the probe system. A procedure can be envisioned where previouslyobtained diagnostic information is used to localize a region where higher resolution may be desired, then an appropriate probe position is determined to maximize resolution at that location. Finally, a PET scan, including the probe, is performed. Another application for the $\mathrm{PET}$ probe is ROI imaging, where the region where high resolution is desired is known a priori and the probe can be directly applied in a fixed geometry, for example brain and breast imaging.

\subsubsection{Breast Imaging}

According to the World Health Organization (WHO), breast cancer is the leading cause of cancer deaths among women aged 20-59 years worldwide ${ }^{2}$. Screening X-ray mammography is the gold standard for breast cancer surveillance which has been credited with reducing mortality by $33 \%$. However, its value is very limited for women with dense breasts. The ability of mammography to detect cancer was found to be $80 \%$ for women with predominantly fatty breasts but only $30 \%$ among women with extremely dense breasts [23]. Also, screening mammography suffers from a high false positive rate $(11 \%$ in the United States) which can incur substantial personal and financial costs [24]. High false positive rates and unnecessary biopsies are partially due to the nonspecific nature of the structural indicators of breast cancer in mammography, such as micro-calcifications [6]. On

\footnotetext{
${ }^{2}$ http://www.who.int/mediacentre/factsheets/fs334/en/
} 
the other hand, staging breast cancer through axillary dissection is often ineffective and traumatic for the patient [6]. Furthermore, there is no practical and accurate imaging technique for monitoring therapy.

The limitations of traditional diagnostic methods have motivated the study of the applicability of other imaging modalities to breast imaging. There is ongoing work in the field using CT [25, 26], MRI $[27,28]$ and PET [29, 30, 31].

The functional information provided by PET is useful since cellular changes associated with cancer can occur well before structural changes such as calcifications appear [6]. As early as in 1989, wholebody FDG PET was studied in its application to breast imaging [32]. In this study an $82 \%$ sensitivity of whole-body PET to breast tumors of $>5 \mathrm{~mm}$ in size was reported. Subsequent studies have reported sensitivities ranging from $80 \%$ to $100 \%$ and specificities ranging from $75 \%$ to $100 \%$, with reports of highest sensitivities coming from studies that included patients with large tumors [33]. FDG has also been studied for use in the monitoring of breast cancer treatment [34]. However, several limitations have prevented PET from being incorporated into standard breast cancer evaluation. Limitations include low sensitivity, inadequate spatial and contrast resolutions for small lesion, as well as high cost and long scan times. Dedicated positron emission mammography (PEM) systems have been developed to overcome some of these difficulties [33, 35, 31]. For improved spatial and contrast resolution, PEM devices use high-resolution detectors in a geometry which increases sensitivity in a ROI by employing a small FOV. However, these are highly specialized systems with a very narrow range of applicability in clinical practice. Furthermore, they would involve a high financial costs to implement on a large scale.

As a means of obtaining high resolution in a reduced FOV, the PET insert geometry offers a practical solution to the limitations of 
using whole-body PET for breast imaging applications. The technique makes use of a clinical scanner and its only requirement is that a limited number of high-resolution detectors to be connected with a, possibly already-existing, system. This application has been proposed using the ring insert concept, employing a partial ring of highresolution scintillation detectors in coincidence with a whole-body scanner $[36,37]$. A prototype of the system has shown improvements in spatial resolution and contrast recovery in a cylindrical phantom [16]. The application of a probe geometry to breast imaging using a more realistic breast phantom will be the subject of matter of chapter 8 of the present work.

\subsection{Summary}

The PET insert concept aims to enhance the spatial resolution of a conventional scanner by placing additional detectors in the FOV. Ring and probe insert geometries have been envisioned. This chapter illustrates how placing the insert detectors closer to the subject and reducing the size of their individual elements creates high-resolution coincidence events. Further enhancement may be obtained by using insert scintillation detectors with one-to-one coupling or solid-state detectors. Various implementations of the PET insert concept are currently under investigation by several groups and have been discussed in this chapter. Finally, a clinical application of the probe geometry has been proposed. A PET probe could provide functional information useful for breast imaging, providing higher sensitivity and resolution than whole-body PET, while offering an alternative to the highly specialized PEM devices. 


\section{References}

[1] Lonsdale, M.N., Beyer, T.: Dual-modality PET/CT instrumentation - Today and tomorrow. European Journal of Radiology $73(3)(2010) 452-460$

[2] Ulzheimer, S., Flohr, T.: Current Technology and Future Developments. In: Multislice CT. Springer, Berlin Heidelberg (2009) $3-23$

[3] Runge, V.M., Nitz, W.R., Schmeets, S.H., eds.: Image Resolution: Pixel and Voxel Size. In:. Thieme, New York (2009) 24-25

[4] Tai, Y.C., Wu, H., Pal, D., O’Sullivan, J.A.: Virtual-pinhole PET. J. Nucl. Med. 49 (2008) 471

[5] Tomic, N., Thompson, C., Casey, M.: Investigation of the "Block Effect" on spatial resolution in PET detectors. Nuclear Science, IEEE Transactions on 52(3) (2005) 599-605

[6] Peng, H., Levin, C.S.: Design study of a high-resolution breastdedicated PET system built from cadium zinc telluride detectors. Phys. Med. Biol. 55 (2010) 2761

[7] Yin, Y., Komarov, S., Wu, H., Song, T.Y., Li, Q., III, A.G., Lee, K., Simburger, G., Dowkontt, P., Krawczynski, H., Tai, Y.C.: Characterization of highly pixelated CZT detectors for sub-millimeter pet imaging. In: IEEE Nucl. Sci. Symp. Med. Imaging Conf. (2009) 2411-2414

[8] Clinthorne, N., et al.: Very high resolution animal PET. J. Nucl. Med. 41 (2000) 20

[9] Clinthorne, N.H., Brzezinski, K., Chesi, E., Cochran, E., Grošičar, B., Honscheid, K., Huh, S., Kagan, H., Lacasta, C., 
Linhart, V., Mikuž, M., Stankova, V., Studen, A., Weilhammer, P., Žontar, D.: Silicon as an unconventional detector in position emission tomography. Nucl. Instrum. Methods A 699 (2013) 216220

[10] Park, S.J., Rogers, W.L., Clinthorne, N.H.: Design of a very high-resolution small animal PET scanner using a silicon scatter detector insert. Phys. Med. Biol. 52 (2007a) 4653

[11] Park, S., Rogers, W., Huh, S., Kagan, H., Honscheid, K., D., B., Chesi, E., Lacasta, C., Llosá, G., Mikuž, M., Studen, A., Weilhammer, P., Clinthorne, N.: Performance evaluation of a very high resolution small animal PET imager using silicon scatter detectors. Phys. Med. Biol 52 (2007c) 2807

[12] Wu, H., Pal, D., O'Sullivan, J.A., Tai, Y.C.: A feasibility study of a prototype PET insert device to convert a general-purpose animal PET scanner to higher resolution. J. Nucl. Med. 49 (2008b) 79

[13] Wu, H., Pal, D., Song, T.Y., O’Sullivan, J.A., Tai, Y.C.: Micro insert: A prototype full-ring PET device for improving the image resolution of a small-animal PET scanner. J. Nucl. Med. 49 (2008a) 1653

[14] Janecek, M., Wu, H., Tai, Y.C.: A simulation study for the design of a prototype insert for whole-body PET scanners. IEEE. Trans. Nucl. Sci. 53 (2006) 1143

[15] Wu, H., Song, T.Y., Pal, D., Keesing, D.B., Komarov, S., O'Sullivan, J.A., Tai, Y.C.: A high resolution PET insert system for clinical PET/CT scanners. In: IEEE Nucl. Sci. Symp. Med. Imaging Conf. (oct. 2008c) 5442 
[16] Keesing, D.B., Mathews, A.J., Komarov, S., Wu, H., Song, T.Y., O'Sullivan, J.A., Tai, Y.C.: Image reconstruction and system modeling techniques for virtual-pinhole PET insert systems. Phys. Med. Biol. 57 (2012) 2517-2538

[17] Huh, S., Clinthorne, N.H., Rogers, W.L.: Investigation of an internal PET probe for prostate imaging. Nucl. Instrum. Methods A 579 (2007a) 339

[18] Zhou, J., Qi, J.: Theoretical analysis and simulation study of a high-resolution zoom-in PET system. Phys. Med. Biol. 54 (2009) 5193

[19] Huh, S.S., Rogers, W.L., Clinthorne, N.H.: An investigation of an intra-operative PET imaging probe. In: IEEE Nucl. Sci. Symp. Med. Imaging Conf. Volume 1. (26 2007-nov. 3 2007b) 552

[20] Qi, J., Yang, Y., Zhou, J., Wu, Y., Cherry, S.R.: Experimental assessment of resolution improvement of a zoom-in PET. Phys. Med. Biol. 56 (2011) N165

[21] Studen, A., et al.: A silicon PET probe. Nucl. Instrum. Methods A 648 (2011) S255

[22] Zhou, J., Qi, J.: Adaptive imaging for lesion detection using a zoom-in PET system. IEEE Trans. Med. Imag. 30(1) (jan. 2011) 119

[23] Mandelson, M., Oestreicher, N., Porter, P., White, D., Finder, C., Taplin, S., White, E.: Breast density as a predictor of mammographic detection: comparison of interval- and screen-detected cancers. J. Natl. Cancer Inst. 92 (2000) 1081-1087 
[24] Brewer, N., Salz, T., Lillie, S.: Systematic review: the long-term effects of false-positive mammograms. J. Natl. Cancer Inst. 92 (2000) 1081-1087

[25] O'Connell, A., Conover, D.L., Zhang, Y., Seifert, P., LoganYoung, W., Lin, C.F.L., Sahler, L., Ning, R.: Cone-beam CT for breast imaging: Radiation dose, breast coverage, and image quality. Am J Roentgenol. 195 (2010) 496-509

[26] Yang, W.T., Carkaci, S., Chen, L., Lai, C.J., Sahin, A., Whitman, G.J., Shaw, C.C.: Dedicated cone-beam breast CT: Feasibility study with surgical mastectomy specimens. Am J Roentgenol. 189 (2007) 1312-1315

[27] Heywang-Kobrunner, S., Viehweg, P., Heinig, A., Kuchler, C.: MRI of the breast: accuracy, value, controversies, solutions. Eur J Radiol 24 (1997) 94-108

[28] Bluemke, D.A., Gatsonis, C.A., Chen, M.H., DeAngelis, G.A., DeBruhl, N., Harms, S., Heywang-Kobrunner, S.H., Hylton, N., Kuhl, C.K., Lehman, C., Pisano, E.D., Causer, P., Schnitt, S.J., Smazal, S.F., Stelling, C.B., Weatherall, P.T., Schnall, M.D.: Magnetic resonance imaging of the breast prior to biopsy. JAMA 292 (2004) 2375-2742

[29] Freifelder, R., Karp, J.S.: Dedicated PET scanners for breast imaging. Phys. Med. Biol. 42 (1997) 2463-2480

[30] Eubank, W., Mankoff, D.: Evolving role of positron emission tomography in breast cancer imaging. Semin. Nucl. Med. 35 (2005) 84 
[31] Shkumat, N., et al.: Investigating the limit of detectability of a positron emission mammography device: A phantom study. Med. Phys. 38(9) (2011) 5176

[32] Minn, H., Soini, I.: 18F-fluorodeoxyglucose scintigraphy in diagnosis and follow up of treatment in advanced breast cancer. Eur J Nucl Med 15 (1989) 61-66

[33] Schilling, K., Conti, P., Adler, L., Tafra, L.: The role of positron emission mammography in breast cancer imaging and management. Applied Radiology 37 (2008) 26-36

[34] Biersack, H.J., Bender, H., Palmedo, H.: FDG-PET in monitoring therapy of breast cancer. European Journal of Nuclear Medicine and Molecular Imaging 31 (2004) S112-S117

[35] Thompson, C.J., Murthy, K., Picard, Y., Weinberg, I.N., Mako, R.: Positron emission mammography (PEM): a promising technique for detecting breast cancer. Nuclear Science, IEEE Transactions on 42(4) (Aug 1995) 1012-1017

[36] Tai, Y.C., Wu, H., Janecek, M.: Initial study of an asymmetric pet system dedicated to breast cancer imaging. Nuclear Science, IEEE Transactions on 53(1) (Feb 2006) 121-126

[37] Keesing, D., Pal, D., O'Sullivan, A., Komarov, S., Wu, H., Tai, Y.C.: System modeling of a doi-capable half-ring pet insert device for breast cancer imaging. In: Nuclear Science Symposium Conference Record, 2008. NSS '08. IEEE. (Oct 2008) 4218-4222 


\section{Chapter 3}

\section{PET PROBE SYSTEM DESIGN \& SIMULATION}

\subsection{Introduction}

The system studied in this work utilizes the probe geometry applied to a full-body, human PET scanner. This specific PET probe system has its origins in the MADEIRA collaboration ${ }^{1}$, a EU-funded project dedicated to minimizing the dose of radiation received by patients undergoing diagnostic nuclear medicine procedures. In the scope of the project there were five independent approaches for achieving this goal, one of which was the development of a PET probe to be combined with a clinical scanner. Work on the MADEIRA probe continues in several interrelated areas: the development of a probe prototype which utilizes silicon ( $\mathrm{Si}$ ) detectors, experimental characterization and optimization studies, simulation studies as well as the development of data processing and image reconstruction software. This chapter presents the system, based on the MADEIRA concept, designed specifically for

\footnotetext{
${ }^{1}$ http://www.helmholtz-muenchen.de/en/madeira/madeira-home/index.html
} 
simulation studies. A discussion of the choice of simulation software is also included.

\subsection{The Clinical PET Scanner}

The scanner geometry chosen for the simulation studies was based on the PET component of the Siemens Biograph 64 hybrid PET/CT system. The Biograph represents a commercially-available, whole-body PET device used in modern hospitals. Its choice as the scanner component of the PET-probe system allows us to test the probe concept on a PET device capable of a spatial resolution typically achievable in clinical practice. The Biograph's PET detectors are based on 4 $\mathrm{mm}$ LSO crystals, allowing for $5.9 \mathrm{~mm}$ (FWHM) transaxial resolution near the center of the FOV. The simulated system was based on specifications from the manufacturer ${ }^{2}$.

\subsubsection{Scanner Geometry}

The Biograph specifications describe block detectors of 169 LSO crystals each. A single crystal has a FOV-facing surface area of $4.0 \times 4.0$ $\mathrm{mm}^{2}$ and a depth of $20 \mathrm{~mm}$. They were arranged in $13 \times 13$ arrays in each detector block. Although not included in the specifications, 0.2 $\mathrm{mm}$ inter-crystal gaps were also introduced to account for the crystal spacing and the axial FOV given in the specifications. Optical insulation between the crystals was not modeled since light-sharing was not included in the simulation. Rather, light-sharing was accounted for in post-simulation processing via the addition of a crystal-identification uncertainty, or block effect.

The block effect was simulated by sharing single interactions in one crystal amongst its neighbors in the block detector. A single

\footnotetext{
${ }^{2}$ activexray.com/pdf/Siemens_Biograph.pdf
} 
event occurring in a specific crystal was given a $50 \%$ probability of being assigned to one of its 8 neighboring crystals. For crystals on the edge of the block, this percentage was reduced as the distribution was amongst fewer neighbors. The $50 \%$ probability was determined empirically to match the scanner resolution specified for the Biograph 64 .

The scanner consists of three rings, each composed of 48 block detectors, with a diameter of $842 \mathrm{~mm}$. The 3 rings create an axial extent of $163.8 \mathrm{~mm}$, consistent with the $162 \mathrm{~mm}$ axial FOV specified by Siemens. The size of the patient port is $70 \mathrm{~cm}$ and was modeled by discarding LORs not passing through this central region.

\subsubsection{Timing and Energy}

The Biograph specifications state a coincidence timing window (CTW) of $4.5 \mathrm{~ns}$. To simulate timing resolution in the scanner, a Gaussian distribution with $2.25 \mathrm{~ns}$ FWHM, half of the CTW, was employed. An energy resolution of $15 \%$, at $511 \mathrm{keV}$, was modeled in the LSO de-

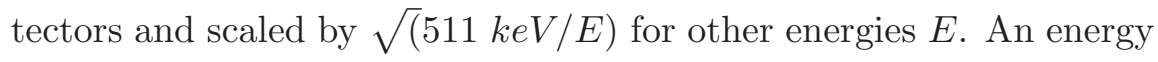
window of 400-650 keV was used for scanner-to-scanner, or "ring", events. Both energy resolution and acceptance window were chosen according to the Biograph64 specifications.

\subsection{The PET Probe}

The MADEIRA PET probe is based on highly-segmented, high-resistivity Si detectors. A number of the researchers involved in the MADEIRA collaboration had previously worked on Si detector applications for PET imaging. As a result, there was considerable experience within the group with this technology $[1,2]$, which was already in an advanced stage of development by the time the MADEIRA collaboration began 
work on the PET probe [3]. The probe design used in the simulation studies utilizes the previous research and implements, using realistic approximations, a device that could be constructed in practice.

\subsubsection{Choice of Probe Material}

Besides the advantage given by its mature stage of development and the high level of related expertise among the MADEIRA collaborators, high-resistivity Si has several properties that make it an attractive choice for the probe material. First, Si detectors have excellent spatial resolution. In a segmented semiconductor detector with individual pixel readout, resolution is limited by pixel size. Si devices with sub-millimeter segmentation are currently manufactured for various applications. Second, Si detectors are relatively compact and sturdy, simplifying mechanical design. Considerable work within MADEIRA focused on this aspect of the probe design [4]. Thin, densely packed detectors are essential for the construction of a probe with DOI capabilities for 3D imaging. Third, superior energy resolution makes $\mathrm{Si}$ useful for event classification, which is especially important in clinical scanners due to the large presence of scattered events. The ability of Si to preferentially Compton-scatter $511-\mathrm{keV}$ photons also makes it potentially useful as a Compton camera [5], where high energy resolution is essential. Using the Compton camera technique, events in which a single annihilation photon undergoes Compton scattering in the probe followed by absorption in the ring, or else multiple Compton scatter in the probe, can be used to add to system sensitivity. Finally, Si detectors can be operated in a magnetic field, allowing future investigation involving PET/MRI to be considered.

The use of other semiconductor detectors, such as germanium $(\mathrm{Ge})$ $[6,7,8]$ and cadmium zinc telluride (CZT) $[9,10,11]$ has been investigated for nuclear medicine applications. Ge and CZT have higher 
atomic numbers than $\mathrm{Si}$ and therefore provide a higher cross-section to 511-keV photons [12]. However, Ge must be cooled to reduce leakage current [12], which makes it less practical for medical applications. CZT detectors, on the other hand, are costly to produce [12]. Si detectors offer a robust and economical alternative to other semiconductor candidates, but with some cost to sensitivity. Their low efficiency is the most serious drawback of Si detectors and requires the use of a thicker detector, or multiple planes, for sufficient stopping power.

\subsubsection{Probe Design}

The probe used in the simulation studies consisted of ten layers of $\mathrm{Si}$ detectors, each being $1 \mathrm{~mm}$ thick and spaced by $1 \mathrm{~mm}$ gaps. Each detector was segmented into an $80 \times 52$ array of $1 \times 1 \mathrm{~mm}^{2}$ pixels, separated from each other by $0.1 \mathrm{~mm}$ gaps $(1.1 \mathrm{~mm}$ pitch). Using a stack of multiple detectors is preferable to using a single thick detector for several reasons. First, a thicker detector has poor timing resolution as there is significant DOI-dependent variation in the detector pulse shape [13] which degrades timing performance. Second, a thinner detector decreases the parallax error due to DOI uncertainty in a single detector. Using multiple layers of Si gives the probe DOI capabilities, making it possible to reduce parallax error in the coincidence events which involve the probe. These events are especially sensitive to parallax degradation due to the proximity of the probe to the object. Finally, using a thinner Si wafer decreases the possibility of multiple scatter in several pixels of a single detector and therefore the occurrence of multiple coincidences. Multiple events in separate detector layers may be used in Compton-camera mode [5]. Multiple Si layers would result in the probe being less compact, however several imaging geometries can be envisioned for a larger probe. 


\section{CHAPTER 3. PET PROBE SYSTEM DESIGN \& SIMULATION}

\subsubsection{Timing and Energy}

The timing response of the $\mathrm{Si}$ detectors is characterized by a delay between the time when a photon interacts in the Si and the time the signal reaches the trigger threshold. The response is a combination of several effects: time-walk, depth-related broadening and electronic jitter. Time-walk is caused by the energy-dependent trigger delay in the readout. Lower energy interactions create a signal which takes a longer time to rise to the trigger threshold. The depth-related broadening is due to a similar effect, but here rise-time is dependent on the depth at which the interaction occurs in the detector [14]. Finally, electronic jitter is noise due to detector electronics.

The model used for the timing response of the simulated probe detectors was based on a study of timing in the MADEIRA probe prototype [15]. In this study, coincidence events from a 511-keV photon source $\left({ }^{22} \mathrm{Na}\right)$ were measured using a 1-mm thick Si detector and a LYSO/PMT assembly (resolution at least 1 ns FWHM) as a timing reference. Due to the excellent timing resolution of the LYSO detector the difference between Si trigger time, $T_{S i}$, and that of the LYSO, $T_{L Y S O}$, is a good estimate of absolute Si trigger delay. Consequently, histograms of relative trigger delay $\left(T_{S i}-T_{L Y S O}\right)$ against energy can be used to represent the Si detector's timing response. The measured coincidence events were plotted in histograms, classified according to the energy deposited in a Si pixel and the Si trigger delay relative to that of the LYSO. Results are shown in figure 3.1.

According to the results of the timing study, the dominant feature in the Si detector's time response is time-walk. This can be observed in figure $3.1 \mathrm{a}$ ) as the movement of the histogram peak towards a longer delay at lower energy depositions [15]. Depth-related broadening and electronic jitter are shown in figure $3.1 \mathrm{~b}$ ) for a $220 \mathrm{keV}$ interaction energy.

Simulations performed by the same group [14] have demonstrated 


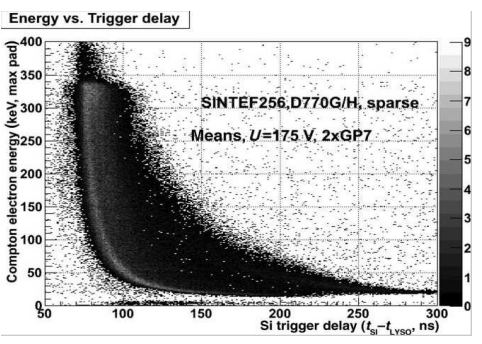

(a)

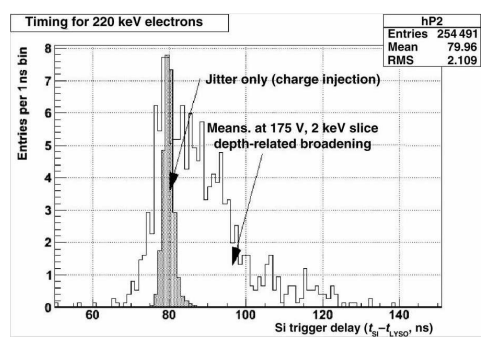

(b)

Figure 3.1: Results from initial experiments to characterize the Si detector timing response. Coincidence events are histogramed according to the energy deposited in a Si pixel and the trigger delay, for a) all energies and b) $220 \mathrm{keV}$ depositions. Figures taken from Studen et al [15].

that 5-10 ns FWHM timing performance is possible if pulse-height corrections are employed. In practice, several methods have been used to improve timing in the detectors. These include increasing bias voltage, thereby accelerating the movement of electrons and holes to the electrodes [16], and subtracting the energy-related delay from each event [17], thereby correcting for time-walk.

For the present study, Si timing response was assumed to be corrected for time-walk and a residual Gaussian spread was assumed [5]. A resolution of 10 ns FWHM was modeled as a conservative estimate taken from the results of the aforementioned simulation study [14]. In chapter 4 of this work, a study is performed to observe the effects of the CTW.

To simulate energy uncertainty in the Si detectors, a Gaussian distribution was used with $1.5 \mathrm{keV}$ FWHM, independent of deposited energy, as determined by a study of the probe prototype [15]. A low energy threshold (LET) of $20 \mathrm{keV}$ was used for probe singles, as 
would be used in experimental application to remove noise from the Si detector. In chapter 4 of this work, a study is performed to observe the effects of the LET in the probe detectors.

\subsection{Simulating the Probe-and-Ring System}

The combined probe-and-ring system was modeled by simulating the two detection systems described above in timing coincidence using the Monte Carlo package GATE (Geant4 Application for Tomographic Emission) [18].

\subsubsection{Geometry}

The proposed system consists of the Biograph 64 and the mobile $\mathrm{Si}$ probe, adaptively positioned according to a given imaging geometry. In general, the probe is placed in close proximity to the region where high resolution is desired. The system, with the probe close to the surface of a Derenzo-like phantom, is shown to scale in figure 14.

\subsubsection{Simulation Tools}

The PET-probe system was modeled using the Monte Carlo package GATE (version 5) [18], which is based on GEANT4 (version 9.2), a platform for the simulation of the passage of particles through matter, written for high-energy physics applications. GATE encapsulates GEANT4's well-validated physics libraries. In addition, GATE provides a PET-specific command set and has itself passed numerous validation studies ${ }^{3}$. The commands provided by GATE make it straight-forward to simulate a conventional scanner such as the Biograph, with its hierarchal detector arrangement of ring-block-crystal,

\footnotetext{
${ }^{3}$ http://www.opengatecollaboration.org/OpenGATEpublications
} 


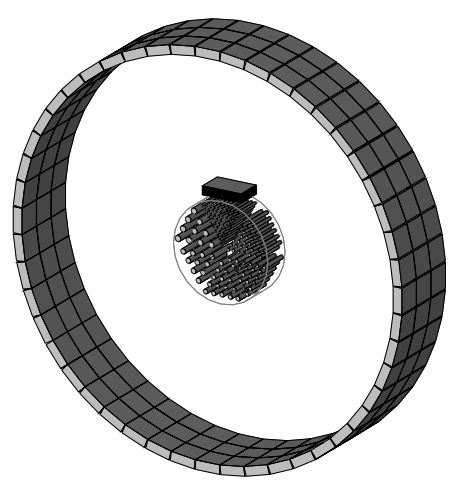

(a)

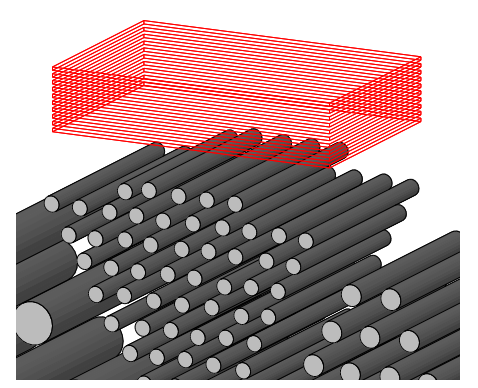

(b)

Figure 3.2: The proposed system with a Derenzo-like phantom, drawn to scale, showing a) the full probe and ring setup and b) a close up of the probe. The Si layers are shown to be transparent in b) and their pixelization is not shown.

simplifying read out of events at each of these levels. Furthermore, GATE is capable of modeling time-dependent phenomena such as detector movement and source decay kinetics.

GATE is intended for simulating more conventional geometries such as SPECT and PET systems and its use for simulating the full ring-and-probe system is a novel application. When simulating the probe geometry special care had to be taken when defining the GATE system and assigning its associated levels to the appropriate physical volumes. The addition of the probe to the typical PET geometry, consisting of rings of detector modules composed of scintillation crystals, required the use of the scanner (or PETscanner) GATE system, which has no fixed geometry.

In GATE, the basic volume elements where information about the interactions is saved must be attached to a crystalSD (SD for 
"sensitive detector") and be part of the chosen system. It is in each of these sensitive volumes that individual interactions, or hits, are summed into a single pulse. These basic detector elements can be grouped into a volume with a single readout common to all, just as crystals in a block detector whose combined signal is read out by a group of PMTs. By attaching this volume to GATE's hierarchal structure of levels, the summing of the signal can be performed using a single command line. The resulting pulse contains the total energy of the block and the location of the crystal with the highest energy deposit (winner-takes-all). Although only an approximation of blockdetector readout, GATE's winner-takes-all approach was used in the Biograph simulation for its convenience. At this stage, the common pulse from each block was used as the GATE singles output. The spectral and temporal resolutions of the Biograph detectors, as well as the energy thresholds, were applied to the singles output using dedicated software.

In the probe, which does not fit into GATE's hierarchal structure, pulses from the Si-pixel level were used as output singles. No common readout was necessary in the probe simulation since every pixel in a Si pad detector is read out individually. Like in the Biograph, the spectral and temporal response of the Si detector, as well as its energy threshold, were applied to the GATE output. No model of dead time was incorporated into the simulation for either detector.

Modeling detector response outside of GATE provided the additional flexibility needed to apply separate time resolutions to LSO and Si detectors. Furthermore, the application of a constant energy uncertainty to events detected in the probe (as explained in section 3.3.3) was made possible. The block effect in the Biograph detectors was applied to the singles output data in the same post-simulation process as time and energy resolutions. 
Because the probe could not be attached to the hierarchy of levels, the GATE coincidence sorter could not be used to create probe-ring coincidence events and a dedicated sorting algorithm was required. Coincidence sorting was performed using the method by which a time window cannot be opened until the previous one has been closed. Random coincidences and those which underwent scatter in the phantom or probe are tagged and included or removed from reconstruction, according to the specific study.

\subsection{Summary}

In this chapter, the PET probe system used in simulation studies was described. The simulated geometry consists of a whole-body clinical PET scanner, based on the Siemens Biograph64, and a layered Si detector probe. Si was chosen due to a number of properties which make the material suitable for such an application. The geometry of the scanner, as well as energy and timing settings, were taken from the Biograph specifications and simulated using GATE, as well as postprocessing of the simulated data. The probe design was based on a prototype constructed by the MADEIRA collaboration. The timing and energy resolutions of the existing device were simulated, but the number of pixels in a single detector and the number of Si layers was modified. GATE was used in a novel way to simulate the probe-andring system. The processing of the simulated data was also briefly described.

\section{References}

[1] Clinthorne, N., et al.: Very high resolution animal PET. J. Nucl. Med. 41 (2000) 20 
[2] Bernabeu, J., Clinthorne, N., Dewaraja, Y., Lacasta, C., Llosá, G., Mikuž, M., Roe, S., Rogers, W., Studen, A., Weilhammer, P., Zhang, L., Žontar, D.: Development of a high efficiency and high resolution compton probe for prostate imaging. Nucl. Instrum. Methods A 527(1-2) (2004) 58-61

[3] Studen, A., Clinthorne, N., Mikuž, M., Kramberger, G.: Timing in thick silicon detectors-an update. Nucl. Instrum. Methods A 579(1) (2007) 83-86

[4] Studen, A., Chesi, E., Cindro, V., Clinthorne, N., Cochran, E., Grošičar, B., Honscheid, K., Huh, S., Kagan, H., Lacasta, C., Llosá, G., Linhart, V., Mikuž, M., Stankova, V., Weilhammer, P., Žontar, D.: Report on the MADEIRA PET probe. In: Nuclear Science Symposium Conference Record (NSS/MIC), 2010 IEEE. (Oct 2010) 1755-1758

[5] Park, S.J., Rogers, W.L., Clinthorne, N.H.: Design of a very high-resolution small animal PET scanner using a silicon scatter detector insert. Phys. Med. Biol. 52 (2007a) 4653

[6] Johnson, L.C., Campbell, D.L., Hull, E.L., Peterson, T.E.: Characterization of a high-purity germanium detector for small-animal SPECT. Phys. Med. Biol. 56 (2011) 5877-5888

[7] Boston, H.C., Boston, A.J., Cooper, R.J., Cresswell, J., Grint, A.N., Mather, A.R., Nolan, P.J., Scraggs, D.P., Turk, G., Hall, C.J., Lazarus, I., Berry, A., Beveridge, T., Gillam, J., Lewis, R.: Characterisation of the SmartPET planar Germanium detectors. Nucl. Instrum. Methods A 579(1) (2007) 104-107

[8] Phlips, B., Kroeger, R., Kurfess, J., Johnson, W., Wulf, E., Novikova, E.: Small animal PET imaging with germanium strip 
detectors. In: Nuclear Science Symposium Conference Record, 2002 IEEE. Volume 3. (Nov 2002) 1438-1442 vol.3

[9] Peng, H., Levin, C.S.: Design study of a high-resolution breastdedicated PET system built from cadium zinc telluride detectors. Phys. Med. Biol. 55 (2010) 2761

[10] Gu, Y., Matteson, J.L., Skelton, R.T., Deal, A.C., Stephan, E.A., Duttweiler, F., Gasaway, T.M., Levin, C.S.: Study of a highresolution, 3D positioning cadmium zinc telluride detector for PET. Phys. Med. Biol. 56 (2011) 1563-1584

[11] Vaska, P., Bolotnikov, A., Carini, G., Camarda, G., Pratte, J.F., Dilmanian, F.A., Park, S.J., James, R.B.: Studies of CZT for PET applications. In: Nuclear Science Symposium Conference Record, 2005 IEEE. Volume 5. (2005) 2799-802

[12] Knoll, G.F.: Radiation Detection and Measurement, Third Edition. John Wiley and Sons, Inc. (2000)

[13] Studen, A., Burdette, D., Chesi, E., Cindro, V., Clinthorne, N., Cochran, E., Grošičar, B., Honscheid, K., Kagan, H., Lacasta, C., Llosá, G., Linhart, V., Mikuž, M., Stankova, V., Weilhammer, Z., Žontar, D.: Performance of the MADEIRA PET probe prototype. In: Nuclear Science Symposium Conference Record (NSS/MIC), 2009 IEEE. (Oct 2009) 3111-3115

[14] Clinthorne, N., Burdette, D., Studen, A., Honscheid, K., Kagan, H., Chesi, E., Huh, S., Lacasta, C., Llosá, G., Mikuž, M., Smith, D., Weilhammer, P.: Timing in silicon pad detectors for compton cameras and high resolution PET. In: Nuclear Science Symposium Conference Record, 2005 IEEE. Volume 5. (Oct 2005) $2868-2872$ 
[15] Studen, A., et al.: Performance of the MADEIRA PET probe prototype. In: IEEE Nucl. Sci. Symp. Med. Imaging Conf. (24 2009-nov. 1 2009) 3111

[16] Studen, A., Burdette, D., Chesi, E., Cindro, V., Clinthorne, N., Cochran, E., Grošičar, B., Kagan, H., Lacasta, C., Linhart, V., Mikuž, M., Stankova, V., Weilhammer, Z., Žontar, D.: Timing performance of the silicon PET insert probe. Radiat Prot Dosimetry 139(1-3) (2010) 199-203

[17] Studen, A., Brzezinski, K., Chesi, E., Cindro, V., Clinthorne, N., Grkovski, M., Grošičar, B., Honscheid, K., Huh, S., Kagan, H., Lacasta, C., Llosá, G., Linhart, V., Mikuž, M., Stankova, V., Weilhammer, P., Žontar, D.: Narrowing coincidence timing window through time-walk correction in silicon ring enhanced PET scanner. In: Nuclear Science Symposium and Medical Imaging Conference (NSS/MIC), 2011 IEEE. (Oct 2011) 266-270

[18] Jan, S., et al.: GATE: a simulation toolkit for PET and SPECT. Phys. Med. Biol. 49 (2004) 4543 


\section{Part II}

\section{PREPARATORY WORK}




\section{Chapter 4}

\section{EVENT}

\section{CLASSIFICATION}

\subsection{Introduction}

In the probe geometry, high-resolution insert-scanner, or "probe", events are added to standard scanner-scanner, or "ring", events to increase the image resolution provided by the PET system. The quantity and quality of the probe events has a great impact on the degree of improvement and the inclusion of these events can even lead to degradation of the images, due to the presence of scattered and random coincidences. The system should be optimized so that the number of true events, both ring and probe, is maximized while keeping the scattered and random events to a minimum. The true events, ring and probe, are those that contribute to image quality, they involve non-scattered photons originating from a single annihilation event. Randoms and scattered events degrade image quality. The settings of the coincidence timing window (CTW) and the low energy threshold (LET) in the Si detectors can influence the quantities of each type of 
event. The optimization of these parameters would involve extensive study beyond the scope of the current investigation, but a general idea of their influence can be obtained by observing the rates of collection of different types of coincidence events. This chapter presents a study of the main types of events, observing how variations in CTW, Sidetector LET and phantom activity influence the quantities of each. The results of these studies aided in determining parameters used in further investigation of the PET probe system. While the study concentrated on probe events, it was also important to consider the ring component, noting the impact of adding probe data.

\subsection{Experimental Method}

To observe the effect of CTW and Si-detector LET on the abundance of various event types, a study was performed by simulating the probe system and a scatter phantom with variable activity. This section defines the types of coincidence events under investigation and includes a description of the simulation study.

\subsubsection{Identifying Types of Events}

The classification of probe events is analogous to that of the regular scanner events presented in section 1.4.1. These events are described in figure 4.1 for a probe system and include: a) true coincidences, b) randoms, c) object-scattered events and d) multiple coincidences in the probe. Multiple coincidences due to a single photon-pair are highly unlikely in the scanner ring due to the 400-keV LET. As the CTW is extended, multiples may occur in the ring due to pile-up. However, the layered structure of the probe and the low LET in the Si detectors may allow for the possibility of a single photon to be detected multiple times in the probe. It may be expected then that 
the contribution of multiples to probe events will be greater than to ring events. Multiple coincidence events could be included in image reconstruction should Compton kinematic processing be employed to select a primary interaction in the probe [1]. Such processing would also be necessary for the use of single-photon coincidences in the probe as Compton camera events. However, such extensions will not be considered here. The multiple coincidences in the PET scanner, along with those measurements involving several interactions in the probe, are excluded from image reconstruction in this work.

\section{Undetected Scatter in Si Detectors}

The probe is an additional scatter medium in the FOV of the PET scanner. Annihilation photons may interact in the Si detectors or in any other of the probe's components, such as the related electronics or support structure. In this study only the sensitive component of the detectors was simulated, therefore only the scatter due to the $\mathrm{Si}$ is considered. Some photons interact in the probe detectors without generating any signal by depositing an amount of energy less than the minimum LET of $20 \mathrm{keV}$ per interaction (applied to reduce noise). These photons may later be detected in the ring or in another of the probe's Si detectors as part of a coincidence event, but their deviation will be relatively small. Figure 4.2 shows a schematic diagram of these two possibilities.

\subsubsection{Simulation Experiment}

The relative amount of the various types of events present in the coincidence data is affected by the choice of the CTW and LET in the Si detectors. Activity levels also have an effect on the rate of these events and therefore should be considered when measuring their contribution. 


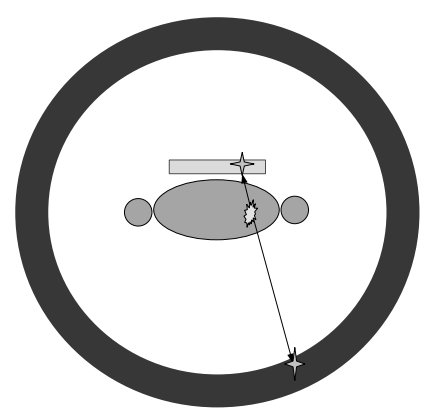

(a)

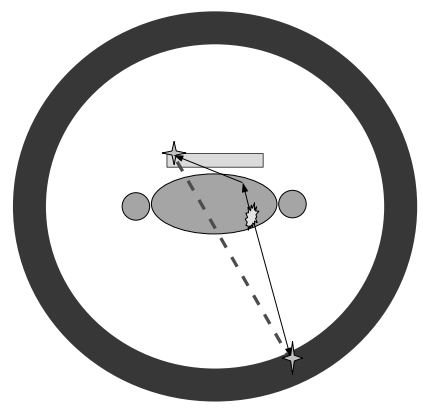

(c)

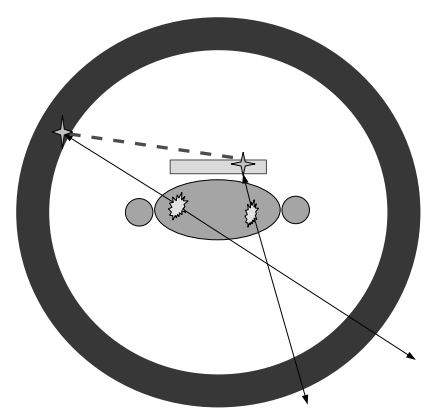

(b)

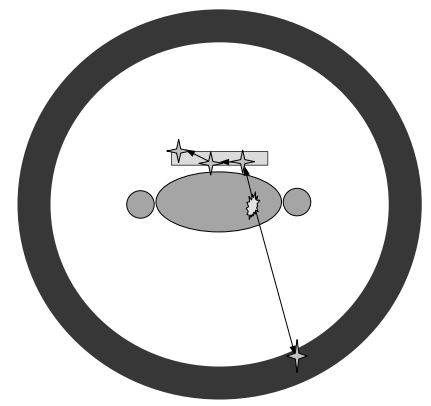

(d)

Figure 4.1: Schematic diagrams of the following types of coincidence events. a) A true probe coincidence. The two detected photons are not scattered prior to detection and originate from the same annihilation event. b) A random probe coincidence. The two detected photons originate from two separate annihilation events. c) A phantomscattered probe coincidence. Here a photon undergoes scatter in the phantom before being detected by the probe (as in the figure) or ring. d) A multiple coincidence with several detections in the probe. In the figure, the three detections in the probe originate from the same photon. 


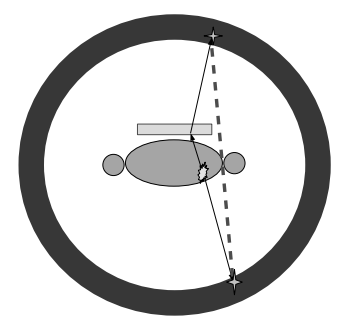

(a)

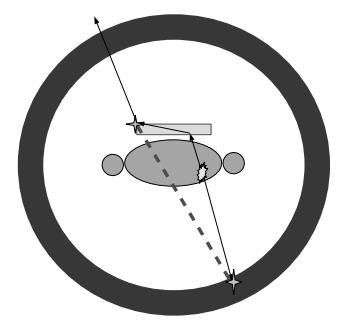

(b)

Figure 4.2: Schematic diagram of an a) ring and b) probe coincidence event involving undetected probe scatter.

To measure the effect of the CTW and the LET, simulations were performed using the NEMA PET Scatter Phantom [2]. This phantom, designed to standardize the measurement of count rate performance of PET devices in the presence of scatter, consists of an axially centered polyethylene cylinder, $70 \mathrm{~cm}$ in length and $20.3 \mathrm{~cm}$ in diameter. The phantom contains a line source, $80 \mathrm{~cm}$ long and $3.2 \mathrm{~mm}$ in diameter, axially centered at a radial offset of $4.5 \mathrm{~cm}$. Simulations of a 10-s acquisition were performed at three different activities: $1 \mathrm{mCi}$, $5 \mathrm{mCi}$ and $10 \mathrm{mCi}$, the middle value representative of the total activity given to a patient during an FDG study. The probe was placed $120 \mathrm{~mm}$ from the center of the FOV, its surface being $8.5 \mathrm{~mm}$ away from the phantom surface and $65 \mathrm{~mm}$ away from the line source. A transaxial view of the probe and phantom is shown in figure 4.3. A required separation of 14 blocks between detected events was imposed to disallow any LOR that does not pass through the Biograph's 70$\mathrm{cm}$ bore. A geometric acceptance cut was imposed on probe LORs by rejecting all measurements that involved a ring detector located beyond the probe position (above the dotted line which is flush with the probe surface in figure 4.3). Counts in these LORs, while clearly 
unrelated to the activity distribution, were tagged and their contribution to randoms and scattered coincidences analysed. In the imaging studies of the forthcoming chapters, no such geometric acceptance criteria was used in the probe since the effect of events in this region may be observed in image-space analysis. However, as the present study only involves the counting of events, such a geometrical disistinction is useful. Coincidence sorting was performed on the processed list-mode singles data as described in section 3.4.2. In order to observe the effects of the CTW, values of 4.5, 10, 15 and 20 ns were used. In PET it is common practice to set the CTW to twice the value of the time resolution of the scanner detectors. The smallest and largest CTW values were chosen to be double the time resolution of the LSO and $\mathrm{Si}$ detectors respectively. Probe LET values of 20, 100, 200, 300, 400 and $500 \mathrm{keV}$ were used and the effect on count rates measured. For the LET studies a CTW of 20 ns was fixed. To help in understanding the LET results, energy spectra of phantom-scattered and non-scattered interactions in the probe were plotted using a 6 -s simulation of the NEMA phantom containing $5 \mathrm{mCi}$.

\subsection{Results}

Coincidence events were classified as ring or probe. For each type, the contribution of true coincidences and randoms was measured, as well as the number of those coincidences containing a scattered photon. Events involving one detection in the ring and multiple detected interactions in the probe were counted separately. The contribution of each type of event is considered as a function of the activity in the phantom and CTW or LET, according to the study. 


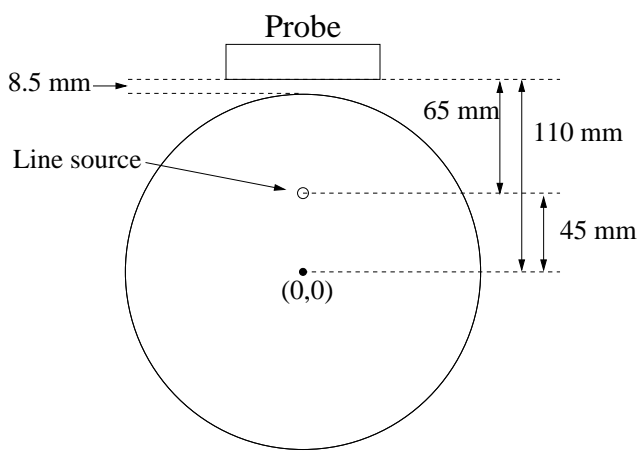

Figure 4.3: Diagram showing the position of the NEMA phantom relative to the probe. The phantom is centred in the FOV of the scanner. The diagram is to scale except for the diameter of the line source, which is doubled for better visualization.

\subsubsection{Effect of Coincidence Timing Window}

The plots describing the effect of CTW on true coincidences, randoms, and those with multiple interactions in the probe are shown below. Uncertainty was calculated considering that the count rates follow Poisson statistics. In this case the magnitude of the resulting error bars are on the order of that of the data-point markers. In the following plots, error bars are included only where they aid in explaining deviations from a clear trend, as in the case of scattered fractions.

\section{True Coincidences}

Figure 4.4 shows how the true coincidence rate varies with the CTW and activity. In figure $4.4 \mathrm{a}$ ) we see that as the CTW is increased there is a reduction of ring sensitivity to true events. At $1 \mathrm{mCi}$ this trend is not significant but increases with activity and becomes significant at $10 \mathrm{mCi}$, where the sensitivity falls to less than half at $20 \mathrm{~ns} \mathrm{CTW}$ compared to the value at $4.5 \mathrm{~ns}$. This trend is expected since the 


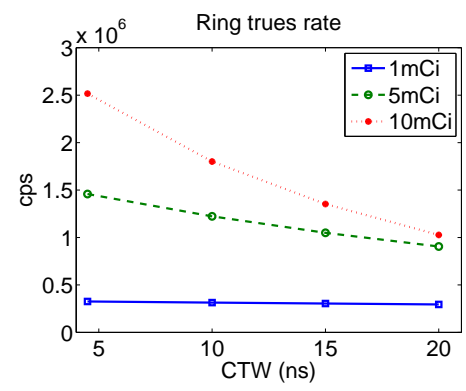

(a)

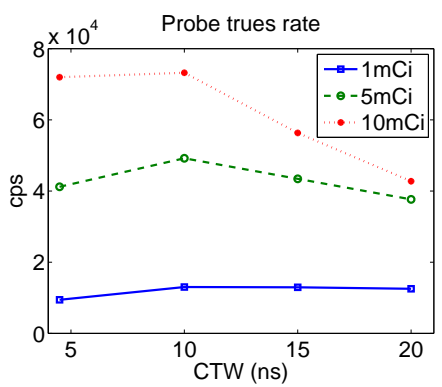

(b)

Figure 4.4: Plots of the true coincidence rate, measured as coincidences per second (cps), for different CTWs and activities for a) ring and $\mathrm{b}$ ) probe events.

probability of multiple detections from different annihilation events (pile up) increases with the width of the CTW. The plot indicates that pile-up begins to affect the coincidence count rate at a CTW lower than 10 ns. Figure 4.4 b) shows that the probe true coincidence rate peaks when using the $10 \mathrm{~ns}$ CTW. This trend is most notable for the higher activities and negligible for the $1 \mathrm{mCi}$ case. For $10 \mathrm{mCi}$ the probe trues rate experiences an overall decrease as the CTW is changed from 4.5 to $20 \mathrm{~ns}$. For $5 \mathrm{mCi}$ there is a small overall drop and for $1 \mathrm{mCi}$ there is a small overall rise. The results indicate that pile-up begins between 4.5 and $15 \mathrm{~ns}$. 


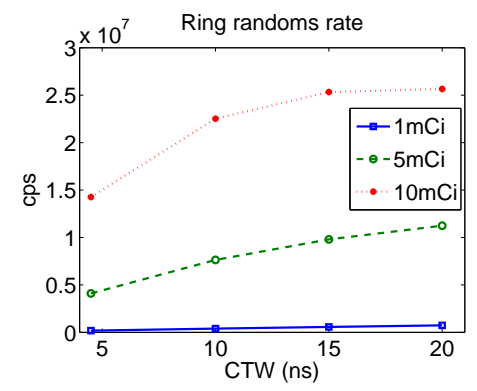

(a)

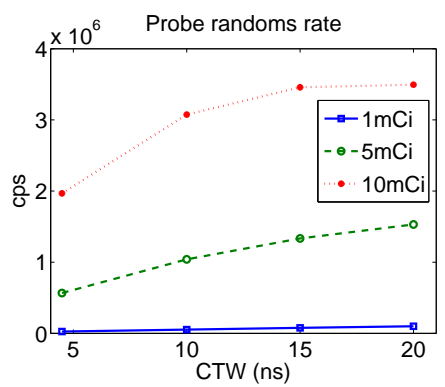

(b)

Figure 4.5: Plots of the random coincidence rate for different CTWs and activities for a) ring and b) probe events.

\section{Random Coincidences}

Figure 4.5 shows how the random coincidence rate varies with the CTW and activity. A similar trend is illustrated for both ring and probe events. An increase is observed, with a higher rate for higher activities, as expected from the relation $N_{R}=2 \tau N_{1} N_{2}$ (equation 5.11). The equation also indicates that the randoms rate should increase linearly with CTW. However, figure 4.5 shows a deviation from this linear behavior, especially at higher activities. This can be explained by pile-up, when an increase in multiple coincidences causes a decrease in those involving two photons.

Figure 4.6 shows the proportion of random coincidences as a fraction of the total number of coincidence events, including trues, randoms and those involving scatter in the object or the probe detectors. The values are high, over $50 \%$ in most cases, especially at high activities. The random fraction is highest in the probe events due to the poor timing resolution in the Si detectors (10 ns FWHM). Extending CTW increases the randoms fraction as expected from figures 4.4 and 4.5. The high randoms fractions among ring events may be explained 


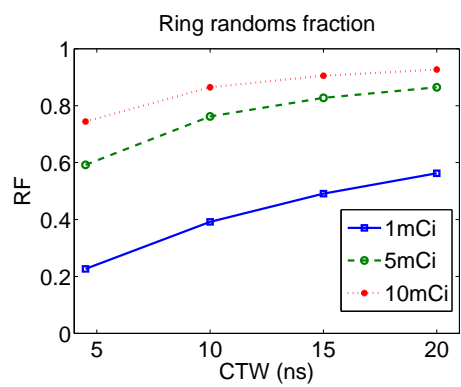

(a)

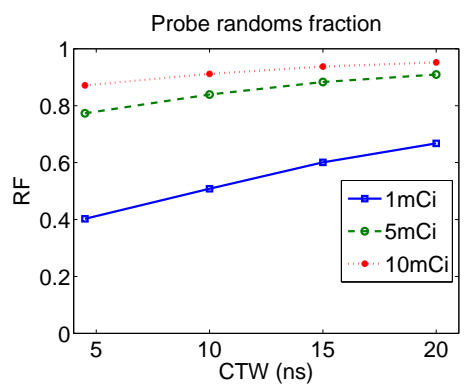

(b)

Figure 4.6: Plots of the random fractions for different CTWs and activities for a) ring and b) probe events.

by the fact that most of the activity in the phantom is located outside of the FOV and no shielding was simulated to prevent the detection of photons from the outside regions. In practice, the clinical Biograph is sandwiched between two lead sheilds to reduce the detection of such events [3].

Counting those random probe events which occured in LORs above the Si device revealed that they would have constituted $56 \%$ of all probe randoms. This high proportion, constant across all activities and CTW values, justifies the implementation of the geometrical acceptance cut on probe LORs.

\section{Multiple Coincidences in Probe}

Figure 4.7 show plots of the rate of events involving one detection in the scanner and multiple detections in various Si pixels. The quantity of these events increases with a larger CTW. However, all of these include at least one non-correlated photon, are therefore classified as randoms and discarded in all remaining studies. 


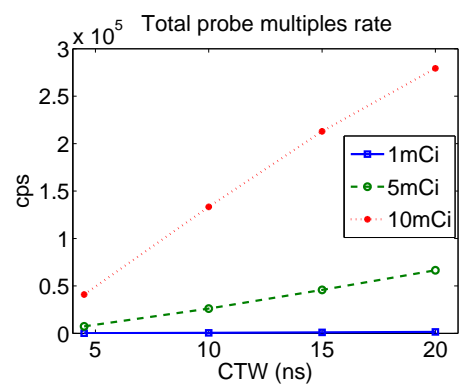

Figure 4.7: Plot of the total probe coincidence rate, with multiple coincidences in the probe, for different CTWs and activities.

\subsubsection{Effect of Probe LET}

In this section the effects of probe LET on the quantity of the various probe event types is illustrated. Plots describing the effect on true coincidences, randoms, those events involving scatter in the phantom, those with undetected scatter in the Si detectors are shown. The count rates of all event types are plotted along with their abundance as fractions of all non-random probe events. Randoms dominate probe coincidence data, as demonstrated in figure $4.6 \mathrm{~b}$ ). When using LETs of 400 and $500 \mathrm{keV}$, beyond the Compton edge for 511-keV photons ( $\sim 340 \mathrm{keV}$, see figure 4.8$)$, there are very few interactions in the probe, often none at all. At such low counts, fractions have little meaning due to large statistical variation and are not plotted.

\section{Energy spectrum}

Figure 4.8 shows the spectra of phantom-scattered and non-scattered probe interactions. For non-scattered events, the mostly flat, slightly concave Compton spectrum for $511 \mathrm{keV}$ photons, ending in the Compton edge at $\sim 340 \mathrm{keV}$, and the photonpeak at $511 \mathrm{keV}$ are clearly identified. The lack of detected events below $20 \mathrm{keV}$ is due to the LET 


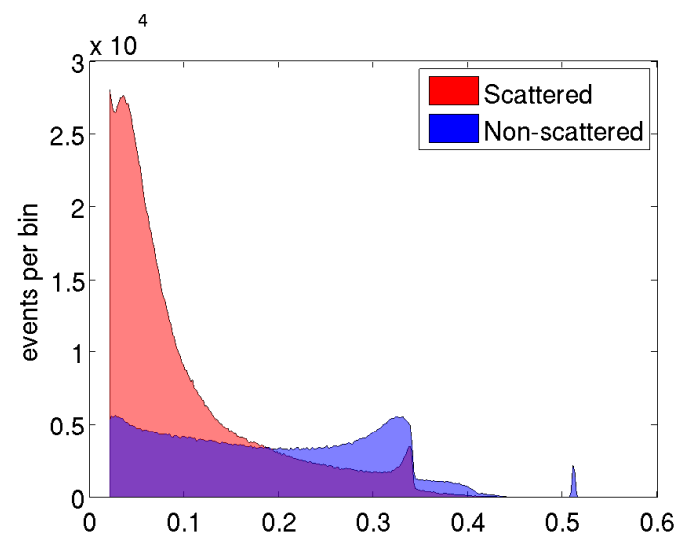

Figure 4.8: Energy spectrum of detections in the probe. The nonscattered events are shown in dark blue and the phantom-scattered events in red.

which is set to that energy. The spectrum of phantom-scattered events is high at low energies and falls quickly, reaching zero at $\sim 420 \mathrm{keV}$, beyond the Compton edge. Although there are some events beyond 400 and $500 \mathrm{keV}$, using these values as a LET in the probe leaves very few probe-ring events to be statistically significant. The preponderance of scattered events at lower energies suggests the possibility that the application of a LET would reduce the scattered fraction.

\section{True Coincidences}

Figure 4.9 shows the effect of LET in the Si detectors on the number of true probe coincidence events. The rate of trues is larger for higher activities and decreases linearly with increased LET, as expected from the energy spectrum. However, the contribution of trues as a fraction of all non-random events (including scattered events) appears to be independent of activity and to increase with LET, up to a maximum 


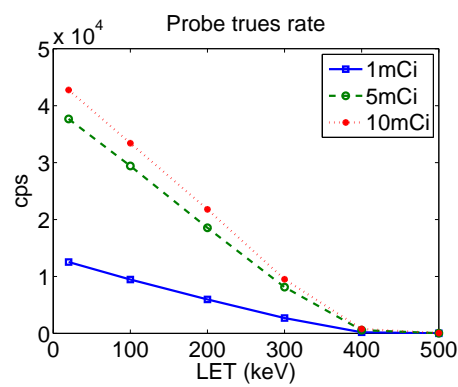

(a)

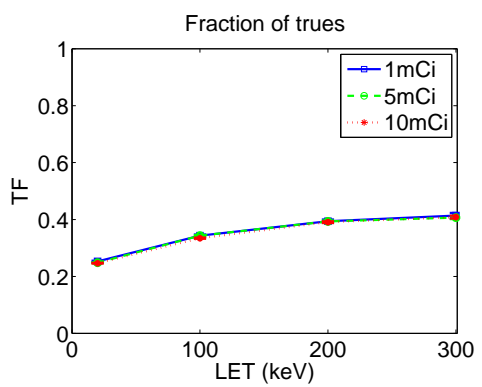

(b)

Figure 4.9: Plots showing the effect of LET in the probe on a) the true probe coincidence rate, measured as coincidences per second (cps), and $b$ ) the $\mathrm{TF}$, for different activities.

at $300 \mathrm{keV}$. Here, the trues fraction (TF) is at a value of about $40 \%$, independent of activity.

\section{Object Scattered Events}

Figure 4.10 shows the object-scattered rate and fraction for probe coincidence events as a function of LET. As with true coincidences, the rate of object-scattered events is larger at higher activities but decreases more sharply with increasing LET than the rate of trues. As a result, the object-scattered fraction (object SF) decreases with LET to a value of $60 \%$ at $300 \mathrm{keV}$. As with the TF, the SF is independent of activity.

\section{Random Coincidences}

Figure 4.11 shows the effect of raising LET on randoms. The randoms rate falls sharply as LET is increased. It does so faster than the trues rate and, as a result, the randoms fraction decreases with increasing LET. 


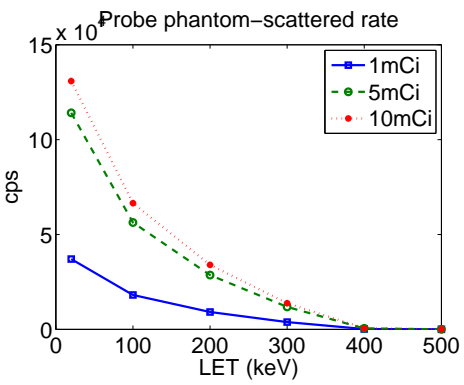

(a)

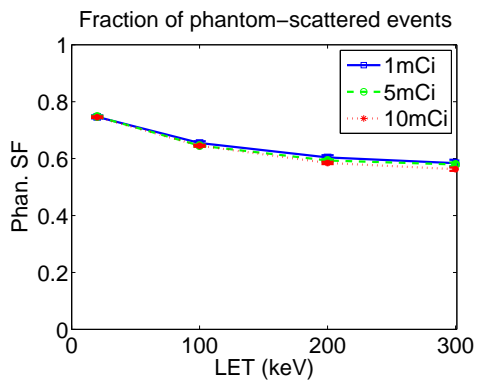

(b)

Figure 4.10: Plots showing the effect of LET in the probe on a) the object-scattered probe coincidence rate and b) the object SF, for different activities.

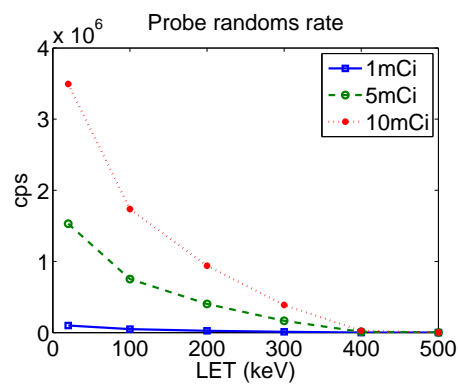

(a)

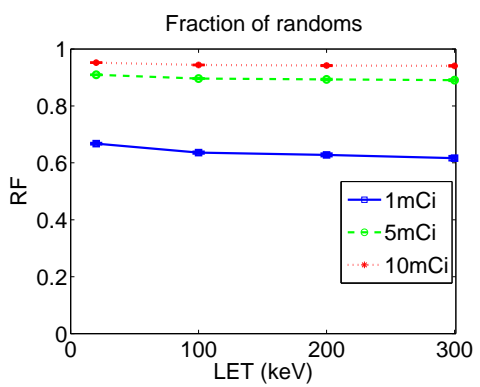

(b)

Figure 4.11: Plots showing the effect of LET in the probe on a) the random probe coincidence rate, measured as coincidences per second (cps), and b) the RF, for different activities.

\section{Undetected Scatter in Si Detectors}

The fraction of probe coincidence events that underwent undetected scatter in the $\mathrm{Si}$ prior to detection in the probe was found to be minimal, between 1 and $2 \%$.

Varying the probe LET above the minimum of $20 \mathrm{keV}$ does not 
affect ring events since the only scatter in the probe that goes undetected occurs under this minimum. It was found that less than $1 \%$ of all ring events are affected by this type of probe scatter, which causes only low-angle deviations in the trajectory of the scattered photon. When probe LET is increased, lower-energy interactions in the probe are excluded from reconstruction, but they are detected nonetheless, if they deposit more than $20 \mathrm{keV}$. If the scattered photon is subsequently detected by the ring, the event is labeled a multiple coincidence and excluded, regardless of the energy deposited by the photon in the $\mathrm{Si}$ detector, as long as this is above the minimum LET.

\subsection{Discussion}

For all further imaging studies in this work, CTW values were determined following the common procedure of setting them to twice the resolution (FWHM) of the detectors in the system. The timing resolution of $2.25 \mathrm{~ns}$ in the LSO detectors was chosen according to this method, given that a CTW of 4.5 ns was quoted in the Biograph specifications, and used in further studies when sorting data from ringonly simulations. Following the same method, for the ring-and-probe system, where the 10 ns time resolution of the Si detectors is the limiting factor, a single CTW of 20 ns was used for both ring and probe events. However, it has been shown in this chapter that a 20-ns CTW causes a significant rise in the randoms fraction in probe and ring coincidence data. It has also been observed that the majority of random probe events (56\%) would only contribute to noise in the area above the probe, where there is no activity distribution, thus effectively reducing the randoms rate. In order to further reduce randoms, two possible approaches may be taken. The first would be to improve the timing resolution of the $\mathrm{Si}$ detectors, allowing for a shorter CTW to 
be used. Although several approaches have been proposed for achieving this aim [4], the present work attempts to reflect the limits of the current timing performance of the probe. The second approach would involve optimizing the coincidence sorting algorithm. A double time-window may be implemented, using $4.5 \mathrm{~ns}$ for ring events and a longer CTW only for probe events. This longer CTW could be determined through a study of the behavior of the noise-equivalent count ratio (NECR) under different CTW values. Both approaches should be studied, the first in the sphere of hardware development and the second in software. Random events can also be compensated during image reconstruction. However, the high rate of coincidences with multiple interactions in the probe, may cause difficulties when using the singles method of randoms estimation which is designed for coincidences involving two detections; alternative methods should be investigated. To minimize the effect of random coincidences observed in this study at higher activities, simulation studies presented in further chapters are limited to low activity, around $1 \mathrm{mCi}$.

An increase in the probe LET reduces the rate of scattered probe events as well as their fraction of the non-random total. Increasing the LET from 20 to $300 \mathrm{keV}$ results in the reduction of object SF of $15 \%$. To a lesser degree, increasing probe LET reduces the RF (by $5 \%$ ). Object SF was found to be independent of phantom activity.

In further simulation studies, a LET of $20 \mathrm{keV}$ is used to increase the system's sensitivity to probe events, whether scattered or not. Using these settings and an activity of $1 \mathrm{mCi}$, the relative contributions of trues, randoms and scattered events is shown in figure 4.12.

\subsection{Conclusions}

A simulation study, using the NEMA scatter phantom containing varying activity, was performed to investigate the effect of CTW and 


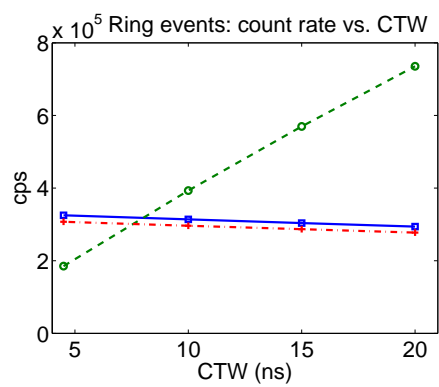

(a)

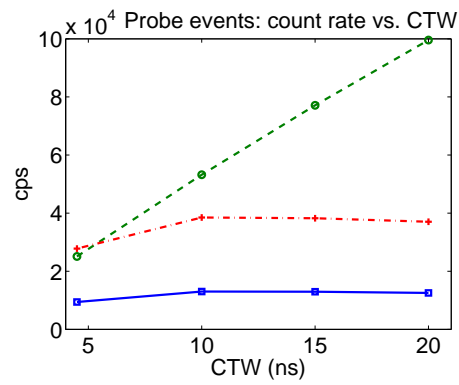

(b)

Figure 4.12: Plots of the relative contributions of trues, randoms and scattered events. a) Shows the results for ring events and b) for probe events, using an activity of $1 \mathrm{mCi}$, probe LET of $20 \mathrm{keV}$. Data points for true events are represented in blue by squares connected by a solid line, randoms in green by circles and a dashed line and scattered events in red by plus signs and a dash-dot line.

probe LET on the quantity of different types of events. High random fractions were observed when using a 20-ns CTW. However, most of the observed random probe events come from spurious measurements which would not contribute to the signal coming from the activity distribution. Nevertheless, the high randoms rate limits the current implementation of the PET probe system to low-activity applications. When using $1 \mathrm{mCi}$ in the phantom, reasonable random fractions were achieved considering that a large portion of the activity was near to, but outside the true coincidence FOV of the scanner [5]. For application to higher activities, work should focus on optimizing the coincidence sorting algorithm, possibly by using a dual CTW.

Raising the LET in the probe has been shown to reduce the scattered fraction of probe events. However, a value of $20 \mathrm{keV}$ was chosen for use in further simulations to increase probe sensitivity while maintaining a minimum LET that would reduce low-energy noise in a real 
probe prototype. The implementation of scatter-reducing LET and the analysis of its effect on image quality is left to future work.

Potentially useful coincidence events involving absorption in the ring and multiple interactions in the probe were observed to be all randoms given the limited simulation statistics. Not a single multiple probe coincidence, amongst the $4 \times 10^{5}$ measured, was due to correlated photons. The low probability of detecting a true multiple probe event justifies the exclusion of these events from reconstruction.

While it can be seen that the probe suffers from increaesd random fractions and scattered coincidences, it is inherently designed as a tool for resolution optimization and its performace as such will be the main focus of further chapters. Although methods for improving the quality of probe events have been proposed, the aim of the studies contained in this work concentrates on utilizing these events at the current state of the system's development, with further improvements left to future work.

\section{References}

[1] Rafecas, M., Böning, G., Pichler, B.J., Lorenz, E., Schwaiger, M., Ziegler, S.I.: Inter-crystal scatter in a dual layer, high resolution LSO-APD positron emission tomograph. Phys. Med. Biol. 48 (2003) 821848

[2] National Electrical Manufacturers Association (NEMA): Performance Measurements of Scintillation Cameras. NEMA Standards Publication No. NU2, Washington, D.C. (2001)

[3] Poon, J.K., Dahlbom, M.L., Moses, W.W., Balakrishnan, K., Wang, W., Cherry, S.R., Badawi, R.D.: Optimal whole-body PET 
scanner configurations for different volumes of LSO scintillator: a simulation study. Phys. Med. Biol 57 (2012) 4077-94

[4] Studen, A., et al.: A silicon PET probe. Nucl. Instrum. Methods A 648 (2011) S255

[5] Simon R. Cherry and James A. Sorenson and Michael E. Phelps. In: Physics in Nuclear Medicine. Saunders, Philadelphia (2003) 


\section{Chapter 5}

\section{IMAGE RECONSTRUCTION TOOLS}

\subsection{Introduction}

The specific characteristics of the additional probe data present a challenge for the development of image reconstruction algorithms. While containing higher resolution information, the probe dataset is incomplete, containing only a limited range of projection angles. Moreover, the angular coverage decreases as a function of the distance from the probe. The resolution of the data is likewise degraded as we move in the direction of the conventional PET detectors, as may be observed in figure 2.6. Should the probe data be reconstructed in isolation, its characteristics would be expressed as a rapid degradation of image quality in areas which are not in the close vicinity of the additional detectors. However, it is worth stressing this extra data is not meant to be used by itself but should be combined with the complete tomo- 
graphic data provided by the conventional PET system. Combining the high-resolution incomplete information with the low-resolution tomographic information is expected to result in an image that has the benefits of both datasets. This combination requires dedicated image reconstruction software that accurately considers the nature of the probe data. In this chapter we investigate the characteristic properties of the probe data, examining the challenges to image reconstruction which they create. Each challenge is provided a solution which contributes to the development of a dedicated image reconstruction algorithm. Wherever appropriate, a study is carried out to demonstrate the character of the data and the enhancement observed when accounting for it during image reconstruction. Although this chapter does not include the main results of the disertation, the software it describes makes all further studies possible.

\subsection{Methods}

In this section, the development of the image reconstruction package for the PET probe system is presented according to how it was developed: as a sequence of system-specific problems and their corresponding solutions. We begin with a closer look at the nature of the probe data.

\subsubsection{Limited FOV}

The limited FOV of the probe is illustrated in figure 5.1. The shaded region represents the area occupied by those probe LORs associated with the potential location of a patient. In practice, LORs not contained in this area could be removed from the data, possibly based on the anatomical information provided by the corresponding CT or MRI scan, thus removing noise related to spurious measurements. It has been shown in chapter 4 that events from this excluded region 


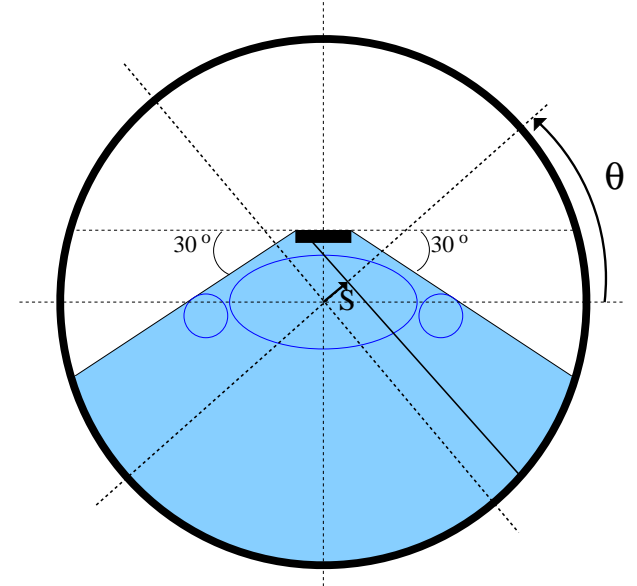

Figure 5.1: A schematic diagram demonstrating the limited FOV of the probe LORs. Data from those detectors without projection information regarding the object being imaged, should be excluded using geometrical considerations. Here this is implemented by a $30^{\circ}$ cut.

dominate the probe randoms rate. However, a situation where there is activity distributed on either side of the probe is also conceivable. It is to allow for such an imaging scenario that the studies presented in this work do not implement any projection-space limitations.

\section{Radon-Space Limitation}

The limited angular coverage of the probe can best be visualized in Radon space. A 2D sinogram was calculated assigning each possible probe LOR with an angle $\theta$ and offset $\mathrm{S}$ according to definitions illustrated in figure 5.1, and binning all axial differences together. We use $1^{\circ} \times 2 \mathrm{~mm}$ bins to form a histogram representing LOR density throughout the FOV. As a result, the sinogram contains probe sensitivity information. The removal of LORs defined by the $30^{\circ}$ limit 
from the sinogram was implemented to observe the effect on the sensitivity sinogram. The choice of a $30^{\circ}$ cut, adapted in this study for illustrative purposes, is indicative of values one might use given the above geometry.

\section{Image-Space Limitation}

To demonstrate the effect of the missing projection angles in image space, images were reconstructed using only the probe events. For this purpose a large Derenzo-like phantom (as displayed in figure 14), $21.4 \mathrm{~mm}$ in diameter, with inserts measuring 12.7, 11.1, 9.5, 7.9, 6.4 and $4.8 \mathrm{~mm}$ in diameter, was simulated.

\subsubsection{List-Mode ML-EM}

The probe coincidences provide a detection response of fan-like geometry, with resolution being highest at the Si pixel surface and decreasing with distance to the resolution of a conventional scanner event at the surface of the scintillation crystal (see section 2.2.2). In addition, this variable-resolution information must be combined with lower-resolution ring data. Such multi-resolution data is problematic when constructing sinograms, since the high-resolution information requires finer sampling than that of lower resolution. Furthermore, the layered structure of the probe causes multiple LORs, each with a different coincidence response, to be assigned to the same sinogram bin. Given these limitations, sinograms were not used to store projection data for image reconstruction for the probe system.

The fine pixelization of the $\mathrm{Si}$ detectors results in an abundance of possible measurements in the probe data. There are 2 to $4 \times 10^{9}$ different LORs in the PET probe system, depending on FOV restrictions. This number may be somewhat reduced by the implementation 
of projection-space cuts. However, the abundance of possible LORs makes storing data in a LOR histogram an impractical alternative to the sinogram due to its size. Furthermore, if the probe were to be moved to multiple positions during a single acquisition, the number of distinct LORs and sinogram bins would increase significantly. We considered it more appropriate to use a list-mode (LM) approach [1] rather than histograms of measurements. Using this method, the summation in the ML- EM algorithm runs through the $\mathrm{N}$ events in the LM data $(l=1, \cdots, N)$. The algorithm is given by:

$$
f_{j}^{k+1}=f_{j}^{k} \sum_{l=1}^{N} a_{l_{i} j}^{\prime} \frac{1}{\sum_{b=1}^{J} a_{l_{i} b}^{\prime} f_{b}^{k}} .
$$

The image reconstructed using this formulation of the algorithm is an estimate of the distribution of emitted counts which are detected [2], rather than of the actual activity distribution (all emitted counts), as in equation 1.20. To obtain an estimate of activity distribution, sensitivity correction must be performed after the final iteration by dividing the image by the sensitivity matrix. Similarly, the system matrix element $a_{l_{i j}}^{\prime}$ is the probability that a detected emission from pixel $j$ is detected in the $i^{\text {th }}$ detector-pair, corresponding to event $l$. Such a system matrix is obtained from the matrix $a_{i j}$ (as used in equation 1.20) but needs to be normalized for system sensitivity. For simplicity (and without loss of accuracy in the definition) we call it $a_{i j}^{\prime}$ in further discussion.

\subsubsection{Multi-Line Siddon}

The probe is conceived as a mobile device, therefore a pre-calculated system matrix is not a practical solution since it would have to be recalculated for every probe position. Furthermore, the abundance of possible LORs in the probe data would make the storage of such a large matrix unpractical (several Gb in storage for each probe position, 
for the probe-only system matrix elements alone). Rather, an on-thefly method of calculation is preferred. In addition, a unique sensitivity matrix is required for each probe location. This calls for an efficient method of sensitivity calculation.

The Siddon ray-tracing algorithm [3] provides a fast method of calculating the exact radiological path through a voxel space, an efficient model for the propagation of $511-\mathrm{keV}$ photons. An accelerated version of the algorithm [4] was used for the on-the-fly calculation of the elements of the system matrix as well as for the sensitivity matrix. Using the Siddon method, each system matrix element $a_{i j}$ is proportional to the intersection length, $l_{i j}$, of a line traced between the detectors of the $i^{t h}$ LOR, through pixel $j$. In the above formulation of the LM ML-EM algorithm, it is emphasized that $a_{i j}^{\prime}$ expresses the probability of detecting an emission from pixel $j$ in LOR $i$ given that the event is detected by the system at all. Expressed mathematically, we insist that $\sum_{i} a_{i j}^{\prime}=1$. Without this condition of detection by the system, the overall probability $a_{i j}$ of detecting an emission for a certain $i-j$ combination is related to the Siddon line length through a proportionality constant $C_{1}$, such that

$$
a_{i j}=C_{1} \cdot l_{i j}
$$

From this expression, $a_{i j}^{\prime}$ is obtained by dividing the overall probability by the system sensitivity as follows:

$$
a_{i j}^{\prime}=\frac{a_{i j}}{\sum_{i} a_{i j}}=\frac{l_{i j}}{\sum_{i} l_{i j}} .
$$

The division by system sensitivity in the system matrix calculations also reduces the effect of high-frequency, pixel-to-pixel sensitivity variations in the reconstructed image.

The difference in spatial resolution between the conventional PET data and the probe data presents a challenge when using the Sid- 
don method. The high-resolution information of the probe requires a finer pixelization of the image space for proper representation. A finer grid, however, would be undersampled by the low resolution ring data should only one ray per detector pair be used in modeling. Furthermore, as we have seen in chapter 2, the probe detection response is fan-like in geometry and, therefore, is of variable resolution over the length of the LOR. As a solution to these problems we use a multi-ray approach [5]. Instead of tracing one Siddon line between the centres of the two detection elements, four evenly spaced points were used (chosen at a depth of one attenuation length in ring crystals and in the central plane of each Si pixel): 16 Siddon lines per detector pair were calculated. The sampling of the detection response is shown in figure 5.2.

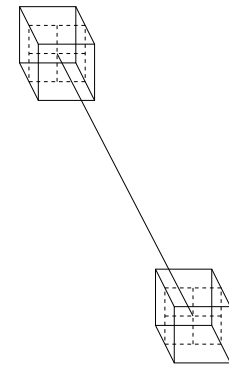

(a)

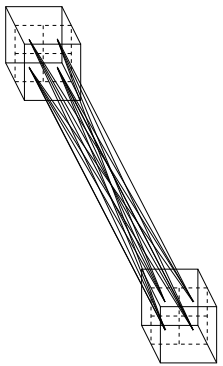

(b)

Figure 5.2: Diagram showing the sampling of the response of a pair of detectors. In a) we see the case of one line per detector pair and b) shows a higher sampling using 16 lines.

Using the multi-line method, the probability $a_{i j}$ is computed using a sum, $l_{i j}^{(D)}$, of all $D$ intersection lengths of the Siddon lines $l_{i_{d} j}$ corresponding to the $i^{\text {th }}$ LOR, through pixel $j$. For a proportionality constant $C_{2}$, the equation becomes 


$$
a_{i j}=C_{2} \cdot l_{i j}^{(D)}=C_{2} \cdot \sum_{d=1}^{D} l_{i_{d} j},
$$

where $d=1, \cdots, D$ is the index that runs over all the $\mathrm{D}$ lines that correspond to detector pair $i$. In the implementation used in this work, $\mathrm{D}=16$. The corresponding system matrix element $a_{i j}^{\prime}$ is again obtained using equation 5.3.

The effect of the increased sampling on reconstructed images can be demonstrated using the sensitivity calculation and reconstructed images of a uniform source distribution. The ring sensitivity was calculated using both 1 and 16-Siddon-line methods to illustrate the effect of increased ray-counts on related image artifacts. The artifacts were further observed in reconstructed images of an extended, 2D square source distribution.

\subsubsection{The Combined System Model}

Although ray-lengths are proportional to detection probability, it is important to recognize that the proportionality constant for a ring event is not the same as for a probe event. Due to differences in the sensitivity and segmentation density of the two detectors used, the intersection length of a Siddon line with an image pixel must be appropriately weighted depending on the type of event: ring or probe. A relative weighting factor, $\beta$, must be calculated a priori for each distinct probe position, such that:

$$
a_{i j}=C_{2} \cdot \begin{cases}l_{i j}^{(16)} & i \in R \\ \beta \cdot l_{i j}^{(16)} & i \in P\end{cases}
$$

where $R$ and $P$ are the sets of ring and probe LORs respectively.

Given the total number of ring and probe coincidence events, $N^{r}$ 
and $N^{p}$, measured using an arbitrary activity distribution $\boldsymbol{\lambda}$, with voxel-wise activities $\lambda_{j}$, the $\beta$ factor can be calculated as:

$$
\beta=\frac{N^{p}}{N^{r}} \frac{\sum_{j=1}^{J} S_{j}^{r} \lambda_{j}}{\sum_{j=1}^{J} S_{j}^{p} \lambda_{j}},
$$

where

$$
S_{j}^{r}=\sum_{i \in R} l_{i j}
$$

and

$$
S_{j}^{p}=\sum_{i \in P} l_{i j}
$$

This expression is valid independently of the chosen, non-zero, distribution $\boldsymbol{\lambda}$, as $\beta$ depends only on probe position. The factor can be estimated by counting $N^{r}$ and $N^{p}$ from a simulation of a known activity distribution $\boldsymbol{\lambda}$. While uncertainty due to counting statistics may be reduced by acquiring a sufficient number of coincidence events, a low activity should be used to avoid the effects of they system's timing performance such as pile-up, especially when using the long CTW required for probe coincidences. For this purpose we simulated a homogeneous, $40 \times 40 \times 40 \mathrm{~mm}^{3}$ cube source in the centre of the FOV, containing $1 \mu \mathrm{Ci}$ of activity, acquiring a sufficient number of events to determine the ratio $\frac{N^{p}}{N^{r}}$ to less than $1 \%$ error. Values of $\beta$ were measured at four probe positions by linearly offsetting the device from the centre of the FOV to positions used in subsequent phantom studies $^{1}$ : 35, 75, 85 and $105 \mathrm{~mm}$.

\footnotetext{
${ }^{1}$ The study with the probe at $85 \mathrm{~mm}$ is not included in this thesis.
} 


\subsubsection{Attenuation Correction}

The probe acts as an attenuating object when placed in the scanner's FOV and interacts with regular PET coincidence events. A method for correcting for this effect must be incorporated into the image reconstruction algorithm. Probe attenuation was accounted for by including a correction factor in the calculation of both the ring and probe system matrix elements. The length $l_{i j}$ of each Siddon line passing through the probe was weighted according to the length of $\mathrm{Si}$ it traversed, $d_{i}$, and the material's total attenuation coefficient $\mu_{S i}$, according to

$$
l_{i j} \rightarrow l_{i j} e^{-\mu_{S i} d_{i}}
$$

To demonstrate the effect of the probe attenuation correction, a homogeneous, 2D square source distribution $\left(20 \times 20 \mathrm{~cm}^{2}\right)$ was simulated and reconstructed both with and without attenuation modeling. Summed line profiles were taken through the image, incorporating the affected region of the phantom. To further demonstrate the effect of the probe attenuation correction, the difference in intensity between an area close to the probe and one further away was compared for both images. Regions of interest (ROIs) of the same width as the probe $(88 \mathrm{~mm})$ and $50 \mathrm{~mm}$ high, leaving a $1 \mathrm{~mm}$ margin from the phantom edge, were chosen at the top and the bottom of the square in the two images. The simulation setup is shown in figure 5.3.

\subsubsection{Random and Scatter Correction}

For selected studies performed in this work, scattered events and random coincidences were compensated for during image reconstruction. Correction for these events can be performed by adding an estimate of their total contribution in the projection operation within the main 


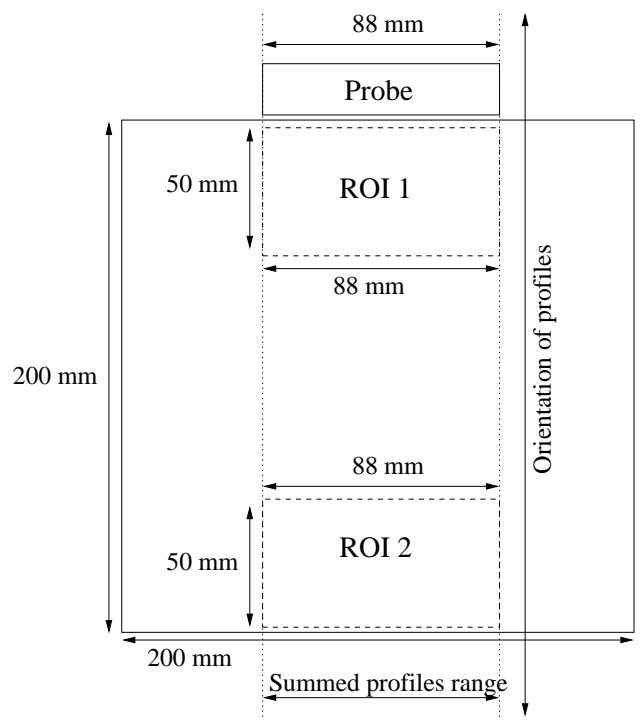

Figure 5.3: The 2D setup used for the probe attenuation demonstration. The size of the square distribution, the probe and the ROIs are drawn to relative scale.

sum of the ML-EM equation [6]. The expression becomes:

$$
\hat{f}_{j}^{k+1}=\hat{f}_{j}^{k} \sum_{l=1}^{N} a_{i j} \frac{1}{\sum_{j=1}^{J} a_{i j} \hat{f}_{j}^{k}+r_{i}+s_{i}},
$$

where $r_{i}$ and $s_{i}$ are, respectively, the total randoms and scatter estimates in the detector-pair $i$. In this work, these correction were applied in all studies where contrast was measured.

\section{Randoms}

As discussed in section 1.4.1, estimates of the randoms rate using the delayed coincidence channel method [7] have a low statistical quality. If the estimate is applied in the projection operation of the ML-EM algorithm, the poor statistical nature propagates to the reconstructed 
image as noise. The delayed coincidence channel method requires the use of variance reduction techniques. The simpler singles rate method requires no such additional procedure. Since singles rates are much larger than coincidence rates, applying the singles rate estimate in the ML-EM algorithm does not introduce as much statistical noise into the reconstructed image and requires no variance reduction. Employing this method for the simulation studies presented in this work, singles rates were taken directly from the simulated data. The number of random coincidences for the $i^{\text {th }} \mathrm{LOR}, r_{i}$, was obtained by multiplying the randoms rate, $R_{i}$, by the simulated acquisition time $\Delta t$. The randoms rate was calculated using the singles-rate equation:

$$
R_{i}=2 \tau S_{i_{1}} S_{i_{2}},
$$

where $\tau$ is the length of the CTW and $S_{i_{1}}$ and $S_{i_{2}}$ are the singles rates in the two detectors corresponding to LOR $i$.

\section{Scatter}

For studies in which scatter correction is applied in this work (see chapter 8), the tail-fitting method described in section 1.4.1 fails since the phantom occupies most of the FOV (and therefore the scatter tails available for fitting are very short). Furthermore, the activity and scatter medium are non-uniform, producing a non-uniform scatter distribution. Since a GATE simulation of the image acquisition system used in this work is already in place, a Monte-Carlo method was a quick way of obtaining a scatter distribution estimate.

An additional simulation of the same phantom as was used in the imaging study, containing similar statistics, was performed. Each photon was tracked and the number of times it underwent scatter in the phantom counted. Two 3D sinograms were constructed, for 
probe and ring data separately, using only those coincidence events which involved a scattered photon. The sinograms were constructed using four parameters: $S$ and $\theta$, as defined in figure 5.2.1; an angle $\phi$, which measures the axial separation of the pair of detectors; and $z$, which measures the axial offset of the line connecting the two. To approximate the low resolution of an emission scan, a course sampling of $(d S, d \theta, d \phi, d z)=\left(4 \mathrm{~mm}, 1^{\circ}, 2^{o}, 2 \mathrm{~mm}\right)$ was used in constructing the sinogram. The scatter estimate, $s_{i}$, was taken as the value in the bin of the scatter sinogram corresponding to the $i^{\text {th }}$ LOR and current event $l$, divided by the number of LORs falling into that bin. This approach assumes that the scatter contribution in all LORs of one bin is similar. In this way, statistical noise was reduced by sharing counts between neighboring LORs.

\subsection{Results \& Discussion}

The results of the studies described in this chapter are presented in this section. A demonstration of the limited FOV of the probe serves to give insight into where improvement can be expected. Results of the multi-line algorithm and attenuation correction demonstrate the effectiveness of these components of the system model.

\subsubsection{Limited FOV}

\section{Probe Sinograms}

The sinograms, shown in figure 5.4, contain probe sensitivity information. The removal of the LORs defined by the $30^{\circ}$ limit (figure 5.2.1) from the sinogram results in sharp cuts in the distribution (figure 5.4 b). Probe data is contained in only a subset of all sinogram bins, whereas the ring LORs from the same system would provide counts 


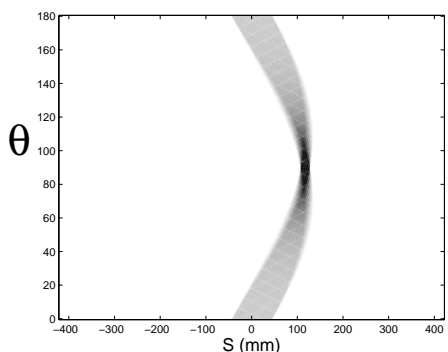

(a)

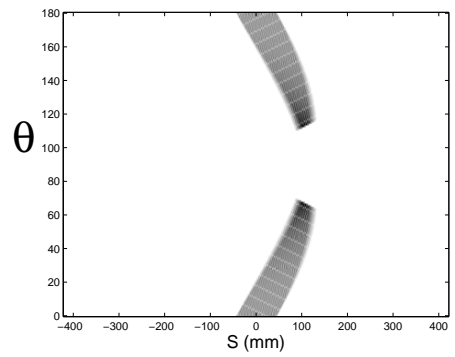

(b)

Figure 5.4: Sinograms showing the limited FOV of the probe in coincidence with the ring using a) all probe LORs, and b) only the LORs passing through the predefined FOV.

to all possible bins. The diagonal lines visible in the sinograms are due to gaps between scanner blocks.

\section{Probe Image}

A reconstructed image of a simulated Derenzo phantom using only probe events is shown in figure 5.5. The degradation due to limited angle data is clearly observed increasing with distance from the probe. The directionality of the resolution produces oblique, fan shaped artifacts. Although the probe data is not meant to be reconstructed in isolation, this reconstructed image is indicative of the information measured by the probe. In the figure, the probe is on the right side of the image where it causes an artifact understood to be due to a coincidence caused by a single photon scattering in the probe then being absorbed in the ring.

\subsubsection{Multi-Line Siddon}

The central transaxial slices from the sensitivity images calculated using the one and 16 Siddon-line methods, shown in figures 5.6 a) and 


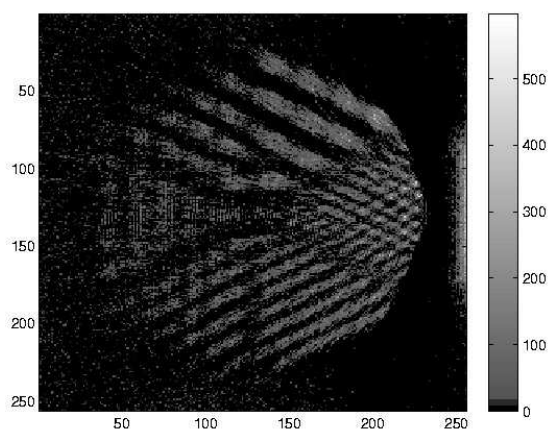

Figure 5.5: The effect of the probe's limited FOV in image space.

b) respectively, display a reduction of artifacts when using 16 Siddon lines. This is further demonstrated by line profiles taken through the centre of the slice (shown in $5.6 \mathrm{c}$ ) and d)). The sharp dip in the centre of the FOV is due to rounding in the implementation of the Siddon algorithm when calculating pixel indices for certain central LORs.

The high frequency fluctuations in the single-line image also appeared in reconstructed images of continuous activity distributions, even when sensitivity correction was applied. Artifacts were strongly reduced when the 16-line model was implemented. In this case, applying sensitivity correction to reconstructed images further reduced sensitivity-related artifacts, as shown in figure 5.7.

For all simulation studies in this work, images were reconstructed using an image space divided into $1 \times 1 \times 1 \mathrm{~mm}^{3}$ pixels, considered sufficient for the resolution attainable using the PET probe system. The 16-line method provided an accurate detection response estimate which allowed for adequate sampling of the image space and reduced artifacts while maintaining reasonable calculation times. Furthermore, a multi-line method is appropriate for modeling the fan shape of the probe LORs. 


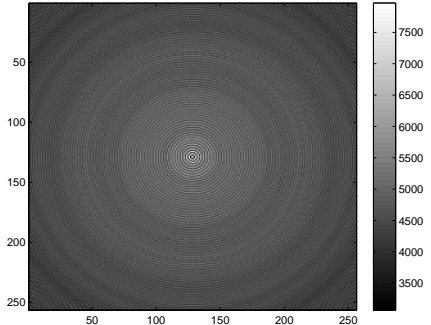

(a)

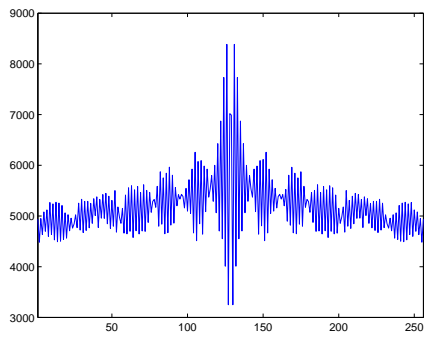

(c)

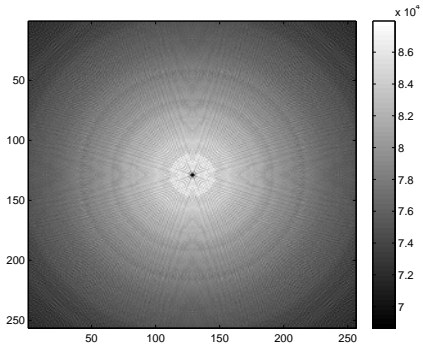

(b)

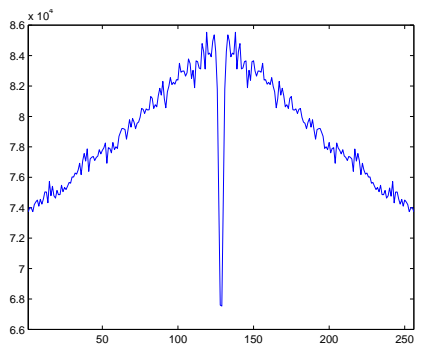

(d)

Figure 5.6: Effect of using the multi-line Siddon method on the ring sensitivity image. The central slice of the image obtained by backprojecting all ring detector pairs using: a) the single-Siddon-line model and b) the 16-line model. Corresponding line profiles thought the centres of the images are shown in c) and d).

\subsubsection{The Combined System Model}

Figure 5.8 shows the values of $\beta$ measured for the chosen probe positions. A near-linear decrease is observed as the probe is moved away from the centre of the FOV. In order to estimate $\beta$ values for other probe positions, more points may be added to the curve and a function fitted. While in this study the probe displacement is in one dimension, this method may be extended to 3D probe movement. Limitations of this method include the dependence of coincidence counting on factors such as pile-up and random coincidences. These effects may be reduced by using a low activity in the distribution $\lambda$. An analytic 


\subsection{RESULTS \& DISCUSSION}

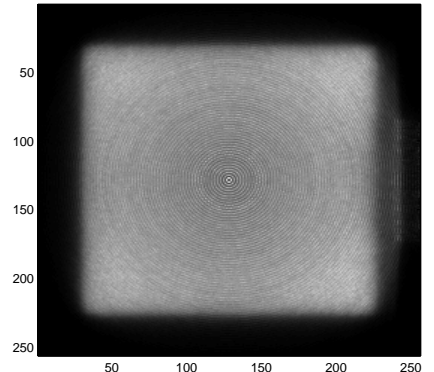

(a)

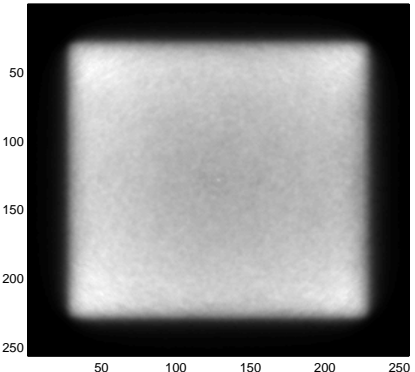

(b)

Figure 5.7: Effect of using the multi-line Siddon method on the image of a 2D square source distribution, reconstructed from ring data only. The image obtained using: a) the single-Siddon-line model and b) the 16-line model.

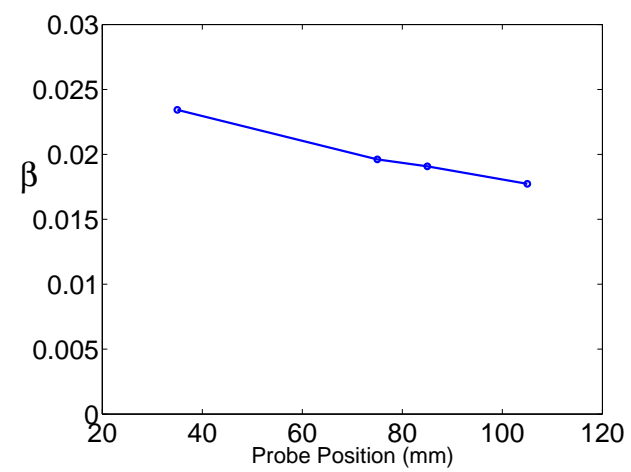

Figure 5.8: The value of $\beta$ measured at several probe offset positions. The probe is moved linearly away from the centre of the FOV, in a direction perpendicular to the Si detector surface.

method using a physical model of the system may be more appropriate and should be the subject of future work. 


\subsubsection{Probe Attenuation Correction}

The effect of probe attenuation correction on images reconstructed using only ring events is shown in figure 5.9. In the image for which attenuation was not considered, 'fading' was observed towards the location of the probe (in this case, near the top of the image). The fading was reduced when attenuation correction was applied. Summed line profiles taken vertically through the central region of the image, directly below the probe position, are shown in figure 5.9 c) and demonstrate that the correction reduces the artifact observed in figure 5.9 a). An intensity difference of $13 \%$ in the non-corrected image was reduced to $0.3 \%$ when the correction was applied, well below the noise level, about $10 \%$ in the corrected image.

\subsection{Conclusions}

This chapter described the development of an image reconstruction software package for the PET probe system. The nature of the probe data was discussed and illustrated using sinograms and reconstructed images. Its unique characteristics were taken into consideration when developing the details which allow the ML-EM reconstruction algorithm to be applied to a clinical PET probe system. The abundance of possible LORs in the data, its variable resolution, as well as the possibility of using multiple probe positions in a single acquisition, motivated the use of the LM version of the algorithm. The mobile probe as well as the abundance of LORs also required on-the-fly calculation of the system matrix and a fast method for computing system sensitivity. For this purpose, the Siddon algorithm was employed for efficiency, its multi-line version providing adequate sampling for the multiple resolution data. Using 16 Siddon lines per detector-pair, provided sufficient sampling for a $1 \times 1 \times 1 \mathrm{~mm}^{3}$ voxels while keeping a 


\subsection{CONCLUSIONS}

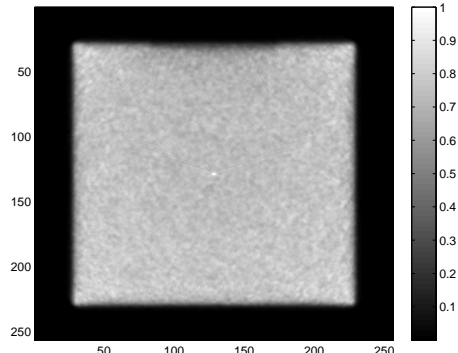

(a)

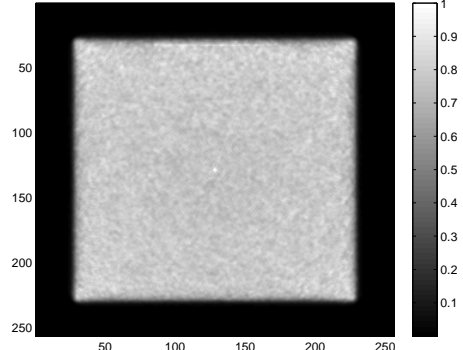

(b)

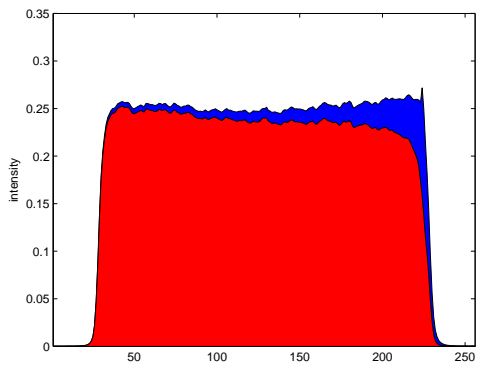

(c)

Figure 5.9: The effect of modeling attenuation due to the probe in the ring image. A homogeneous source distribution is reconstructed a) without and b) with probe attenuation modeling. A comparison of summed line profiles from the area under the probe, taken vertically, perpendicular to the probe surface is shown in c). The difference is seen as the darker shade.

reasonable computation time. A weighting factor $\beta$, which relates the ray-length of the two event types to the relative probability of detection, was introduced and a method of estimating it, based on Monte-Carlo simulation, was presented. An analytical method for $\beta$ calculation may be the subject of future work. Finally, methods for correcting for probe attenuation, probe and phantom scatter, and random coincidences were presented. The probe attenuation correction 
was shown to reduce artifacts related to this effect in the reconstructed image. As described in the Experimental Supplement at the end of the document, software based on the methodology developed in this chapter has also been appllied to several experimental PET prototypes which utilize high-resolution Si detectors.

\section{References}

[1] Barrett, H., White, T., Parra, L.: List-mode likelihood. J. Opt. Soc. Am. A 14 (1997) 2914

[2] Shepp, L.A., Vardi, Y.: Maximum likelihood reconstruction for emission tomography. Medical Imaging, IEEE Transactions on $\mathbf{1}(2)$ (Oct 1982) 113-122

[3] Siddon, R.L.: Fast calculation of the exact radiological path for a three-dimensional CT array. Med. Phys. 12 (2) (1985) 252

[4] Jacobs, F., Sundermann, E., De Sutter, B., Christiaens, M., Lemahieu, I.: A fast algorithm to calculate the exact radiological path through a pixel or voxel space. Cit. Journal of Computing and Information Technology 6(1) (1998) 89-94

[5] Aguiar, P., Rafecas, M., Ortuno, J., Kontaxakis, G., Santos, A., Pavia, J., Ros, D.: Geometrical and Monte Carlo projectors in 3D PET reconstruction. Med. Phys. 37 (2010) 5691

[6] Cheng, J.C., Rahmim, A., Blinder, S., Camborde, M.L., Raywood, K., Sossi, V.: A scatter-corrected list-mode reconstruction and a practical scatter/random approximation techniche for dynamic PET imaging. Phys. Med. Biol. 52 (2007) 2089-2106 
[7] Brasse, D., Kinahan, P., Lartizien, C., Comtat, C., Casey, M., Michel, C.: Correction methods for random coincidences in fully 3D whole-body PET: impact on data and image quality. J Nucl Med. 46 (2005) 859-67 


\section{Part III}

\section{SIMULATION STUDIES}




\section{Chapter 6}

\section{SYSTEM SPATIAL RESOLUTION}

\subsection{Introduction}

The main aim of the high-resolution $\mathrm{Si}$ probe is to provide improved spatial resolution in a limited ROI. Therefore, the first set of simulation studies will investigate the extent of this improvement.

In section 1.4.2, an empirical formula was presented for calculating the resolution of a cylindrical scanner as a function of a number of variables. Section 2.2 examined more closely the influence of detector size and placement on the intrinsic resolution of a detector pair. It was clear from these discussions that the addition of a finely-pixelated probe in the FOV of a clinical scanner would provide an advantage to resolution in the reconstructed image. However, many factors that would determine the degree of this advantage remain to be explored.

Probe and ring coincidences come in different proportions, depending on the sensitivity of the system to each type of event. This proportion is an important factor in determining the resolution of the 
images. The ability of the reconstruction algorithm to combine the two event types and to correctly model the detection response of each also has an important effect on image resolution. The block effect, which affects probe events differently than it does ring events, is another factor which should be studied. Section 2.2 illustrated how the resolution of the probe data varies across the FOV and section 5.3.1 described the spatial variation of the abundance of this data. Therefore, it is expected that the image resolution will also be spatially variant. Furthermore, effects such as timing and energy resolution, which affect randoms and scatter, may also affect image quality.

In this chapter, detailed Monte Carlo simulation studies of several activity distributions are presented to explore the resolution capabilities of the PET probe system under several different scenarios. A preliminary study using ideal events only was performed to investigate the maximum achievable resolution of the two event types. Next, a more realistic simulation of a grid of point sources, excluding object scatter and block effect, illustrates how the resolution improvement brought about by the probe varies over the FOV when these effects are ignored. A subset of the points from the grid was re-examined to observe the impact of adding those effects previously ignored. Finally, a small Derenzo-like phantom was simulated to observe the effects of the probe on a commonly used resolution phantom.

\subsection{Methods}

In section 2.2, the general concept of image resolution is described. In practice, this quantity can be estimated for a specific imaging system using a Monte Carlo simulation of the acquisition of point-source data and observing the response of the system. Below, we present three studies using point sources and describe methods of estimating resolution. In addition, the study of the Derenzo-like phantom is presented. 


\subsubsection{Estimating Resolution}

One method used for estimating transaxial resolution is based on sinograms of point-source data. A line profile taken across the curve that a single point source traces through a 2 -D sinogram, at a single $\theta$ value, gives the corresponding projection of the PSF of the system. By integrating along the length of the curve, all projections are summed, giving an overall resolution estimate for that location in the FOV. However, this method does not give any indication as to the shape of the PSF, which can be asymmetric. For this purpose, the individual projections of the PSF should be considered. These can be taken from the sinogram, as described above, or calculated from the reconstructed image of the point source. The image-based approach involves taking projections through the reconstructed point source in the image and has the advantage of including the effect of the reconstruction algorithm. However, when reconstructing a point source using ML-EM with no background activity, the spatial resolution is artificially enhanced due to an implicit non-negativity constraint. In addition to the artificial enhancement of resolution, cold areas surrounded by activity show positive bias when reconstructed using the algorithm [1]. These effects can be remedied by simulating the point sources over a uniform background which is subtracted prior to analysis [2]. The image profile method can also be used for estimating axial resolution.

\subsubsection{Initial Sinogram-Based Estimates}

In a preliminary study, the transaxial spatial resolution of the probe data was compared with that of the conventional scanner. For this purpose, a distribution of four point sources, of $10 \mu \mathrm{Ci}$ and with $(x, y)$ coordinates $(0,20),(50,70),(-90,10),(2,-20) \mathrm{mm}$, was simulated with the probe located at $(0,21) \mathrm{mm}$ and the origin at the center of the FOV. As an initial study, in order to asses the potential improvement 
in image resolution offered by the probe, simulations were performed without time or energy uncertainty. Annihilation photons were simulated back-to-back, in air. Random coincidences and those with undetected scatter in detectors were also removed to isolate those events with potential to offer maximum spatial resolution. In this way, it was possible to investigate improvement independent of sources of degradation. 2D sinograms were generated for both ring and probe datasets by compressing the data in the axial direction. Projection profiles through one of the points were integrated for all projection angles $\theta$, giving an estimate of resolution at that position as a $1 \mathrm{D}$ PSF.

\subsubsection{Variation Across the FOV}

To expand on the initial resolution study, an additional simulation was performed to investigate the spatial variation of the enhanced resolution and to examine how it is affected by the combination of the two data sets using the dedicated image reconstruction package.

The high-resolution probe LORs are fan-shaped, as they are narrower close to the probe and wider at the PET scanner perimeter. The angle and shape of the fan-like LORs vary, depending on the location and orientation of the two elements involved in detection. The probe data will lead to a PSF which is shift-variant, and non-isotropic, it is expected that this characteristic will be partially conserved when the data is combined with that from the conventional PET scanner. We have studied the varying shape of the PSF in the $x-y$ plane by considering the central transaxial plane of the FOV. For this purpose, a grid of $20 \times 20$ point sources was simulated. The plane of sources was simulated over a low background, which was also simulated separately, reconstructed in isolation and subsequently subtracted before analysis of the point-source grid. The points were spaced by $10 \mathrm{~mm}$ in the $\mathrm{x}$ and $\mathrm{y}$ directions and contained $10 \mu \mathrm{Ci}$ of activity each. The 


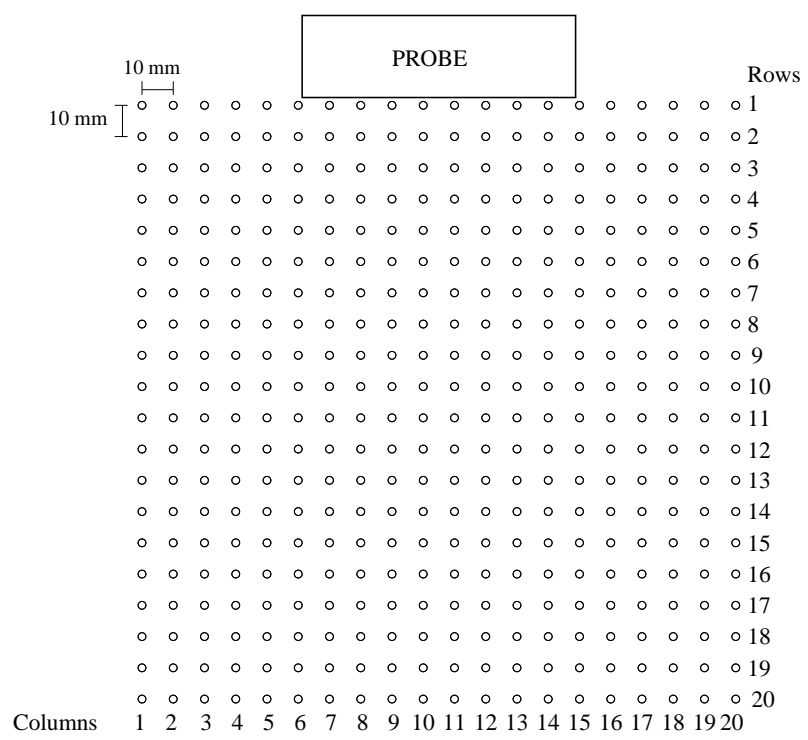

Figure 6.1: The grid of positions at which the point-spread function was calculated. The diagram is to scale.

background was a $200 \times 200 \mathrm{~mm}^{2}$ overlapping plane of $1 \mathrm{mCi}$, resulting in a $0.025 \mu \mathrm{Ci} / \mathrm{mm}^{2}$ concentration. Figure 6.1 shows the location of the point sources and numbering scheme used in analysis. To estimate uncertainty, three realizations of the simulation were performed, each containing 16 million coincidence events. Images were reconstructed on a $1 \times 1 \times 1 \mathrm{~mm}^{3}$ voxel grid using all measurements (including those that did not fit in the restricted FOV, shown in figure 5.1). Backto-back $511 \mathrm{keV}$ gamma photons were simulated in vacuum with the probe in the FOV. To obtain the ring-only image, probe events were not used and probe attenuation correction applied. The block effect was not simulated.

Reconstruction was terminated at 20 iterations of the ML-EM algorithm (see convergence study in section 6.3.2 ). Random coinci- 
dences were included in the reconstruction but not corrected for. The width of the projection profile was measured for each point source in directions perpendicular and parallel to the probe surface. A $10 \times$ $10 \times 10 \mathrm{~mm}^{3}$ region around each point source was considered, and by summing over two of the dimensions, a one dimensional projection of the PSF was obtained. This was conducted over two perpendicular directions to offer insight into the probe's contribution to resolution enhancement in two different directions. Resolution was quantified by calculating the FWHM of the projection profiles, using linear interpolation.

\section{Testing for Convergence}

In order to test the number of iterations required for the PSF to converge, two $10 \mu \mathrm{Ci}$ sample points were resimulated on low-activity background patches of $1 \times 1 \mathrm{~cm}^{2}$, each containing $2.5 \mu \mathrm{Ci}$ in order to maintain the activity concentration ratios of the full-grid study. The chosen points were taken from column 10, rows 2 and 20, to observe convergence in an area close to the probe as well as far away from it. Images were reconstructed and resolution measured at each iteration using the same method as for the the complete grid. Resolution was calculated for both the ring-only image and that including the probe data.

\subsubsection{Addition of Degradation Effects}

The point-source study was repeated in more realistic conditions on a subset of point locations in order to confirm the validity of the full-grid study. The effects of positron range, photon acollinearity, object and detector scatter as well as the block effect and that of probe attenuation on counting statistics were included. In addition, axial resolution 
was measured as it varied with distance from the probe. Column 10 was simulated in a $200 \times 200 \times 20 \mathrm{~mm}^{3}$ rectangular container of water using $10 \mu \mathrm{Ci}$ fluorine- 18 point sources and $1 \mathrm{mCi}$ of fluorine- 18 in the background. Two separate simulations were performed: with and without the probe. Images were reconstructed using 20 iterations of the ML-EM algorithm and the width of the projection profile was measured for each point in directions parallel to the probe surface (in the axial and transaxial directions) and perpendicular to it.

\subsubsection{Resolution Phantom Study}

Simulations of a small Derenzo-like hot-spot phantom, containing an activity concentration of $0.108 \mathrm{mCi} / \mathrm{cc}$, were performed to demonstrate the effect of the probe on the PET scanner's spatial resolution using a common imaging task. The Derenzo-like phantom, with inserts measuring 4.8, 4.0, 3.2, 2.4, 1.6 and $1.2 \mathrm{~mm}$ in diameter, was simulated with the probe $4 \mathrm{~mm}$ away from its surface $(35 \mathrm{~mm}$ away from the centre of the FOV), for two distinct orientations (configurations $\mathrm{A}$ and $\mathrm{B}$ as shown in figure 6.2). Simulations were performed which included the effect of positron range and photon acollinearity, block effect as well as detector time and energy resolutions. Object and detector scatter events, as well as randoms, were present in the coincidence data but not corrected for. Images were again reconstructed using a $1 \times 1 \times 1 \mathrm{~mm}^{3}$ pixel grid, stopping reconstruction at 20 iterations.

\subsection{Results}

In this section we show the results of the various resolution studies performed and analyze the features of the PET probe system's resolution response. A discussion of the implications of the increased resolution is also included. 


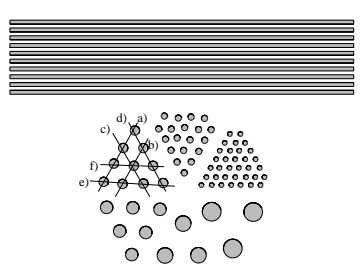

(a)

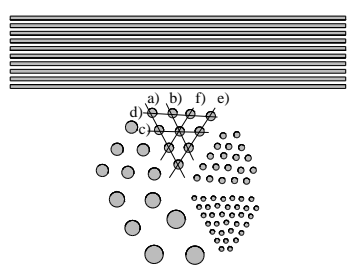

(b)

Figure 6.2: Diagrams showing the two orientations of the small Derenzo-like phantom relative to the probe, labeled (a) A and (b) $\mathrm{B}$ for future reference. Labeling of lines through the phantom is for reference in analysis.

\subsubsection{Estimates from Sinograms}

Separate 2-D sinograms, containing ring and probe data, of the group of four point sources are shown in figure 6.3. It can be seen that while all four points appear in the ring sinogram and can therefore be reconstructed from the PET ring data, only one of them can be clearly seen in the probe sinogram, and therefore lies in the FOV of the probe. This point is located at the detector's surface. A second point is barely visible at very low and very high $\theta$ values. This point is located far away from the probe $(400 \mathrm{~mm})$ but directly below it. The findings are in agreement with the probe sensitivity sinograms obtained in 5.3.1. The noise visible in the probe sinogram may be due to randoms or events where one of the annihilation photons is scattered and detected in the probe and subsequently absorbed in the ring.

Figure 6.4 shows a comparison of the 1D PSF for the ring and probe datasets for the point which most clearly appears in the probe sinogram. The curves are normalized to the number of counts in each peak for easier comparison. The full-width at half-maximum (FWHM) 


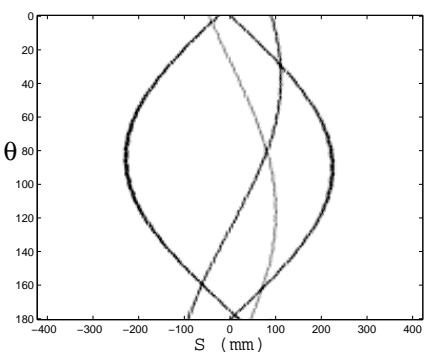

(a)

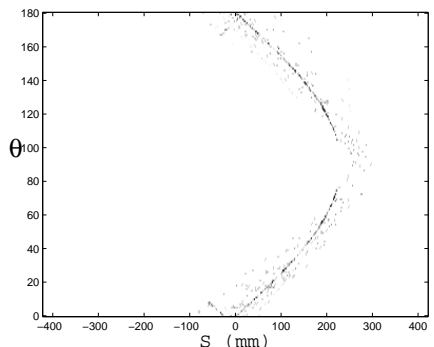

(b)

Figure 6.3: Sinograms of the four point sources containing a) ring coincidences only, and b) probe coincidences only.

of the ring data is $5.5 \mathrm{~mm}$ while for the probe events it is $2.7 \mathrm{~mm}$. The full-width at tenth-maximum also reduces from $7 \mathrm{~mm}$ for ring events to $4 \mathrm{~mm}$ for probe events. The improved spatial resolution of the probe provides a reduction of about half in the width of the PSF.

\subsubsection{Testing for Convergence}

Plots of measured resolution as a function of iteration number are shown in figure 6.5. The resolution is seen to converge to relatively stable values at about 10 iterations for both point-source locations. Therefore, 20 iterations, as used in the resolution studies, is sufficient for obtaining accurate results in the whole ROI. It is also noteworthy that with the addition of the probe data, resolution takes longer to converge. This is most evident in in figure 6.5 a) and is expected due to the increased resolution and sensitivity brought by the probe in this region. 


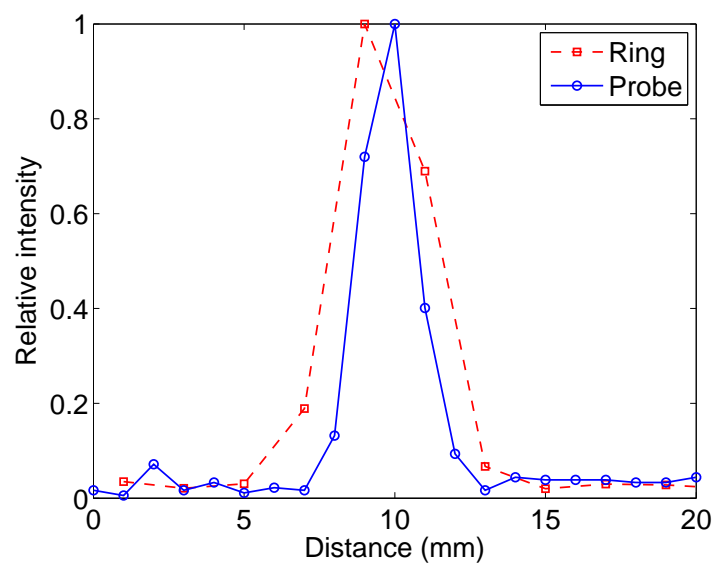

Figure 6.4: 2D PSFs of the ring data (red dashed line) and the probe data (solid blue line). A shift in the position of the maximum is notable here and is further discussed in section 6.4.

\subsubsection{Variation Across the FOV}

Each of the three simulations of the grid of point sources contained $1.1 \times 10^{7}$ coincidence events (excluding the background) of which $15 \%$ were probe events. Plots of the measured FWHM for representative point sources, are shown in figure 6.6 (resolution in the direction perpendicular to the surface of the probe varying over rows) and figure 6.7 (resolution parallel to the surface of the probe varying over columns). The results show improvements in resolution in limited regions of the FOV, the location of which depends on the direction of the projection profiles. We observe an improvement of up to $40 \%$ in the perpendicular direction and up to $30 \%$ in the parallel direction.

\subsubsection{Addition of Realistic Effects}

In re-simulating Column 10 under more realistic conditions, $4.4 \times$ $10^{6}$ coincidence events were acquired by the Biograph system alone 


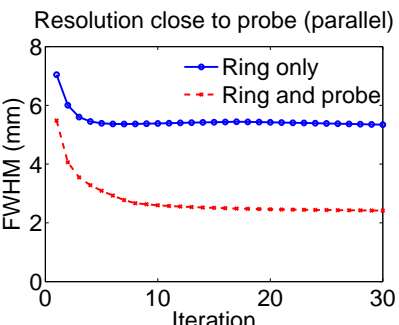

(a)

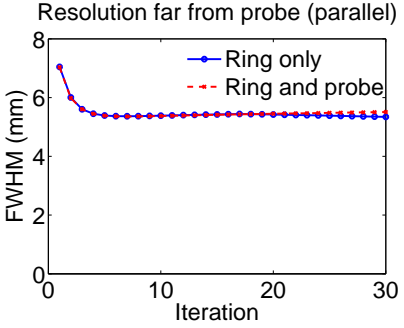

(c)

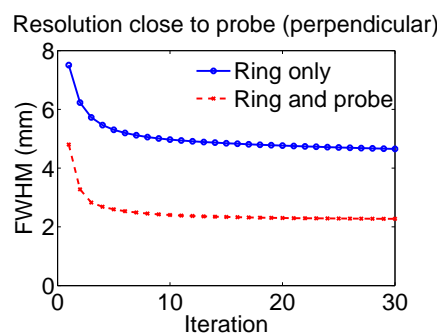

(b)

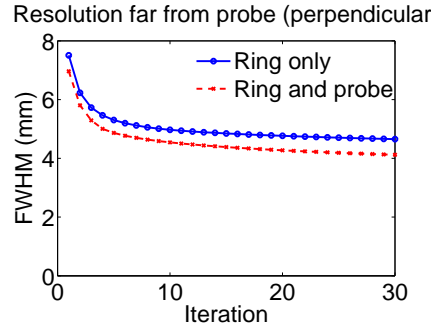

(d)

Figure 6.5: The transaxial FWHM at two positions and in two directions: $1 \mathrm{~cm}$ away from the probe surface, a) parallel and b) perpendicular, and $19 \mathrm{~cm}$ away from the probe surface, c) parallel and d) perpendicular. Data for the Biograph alone is represented by the blue solid line, while that of the probe system is represented by the red dashed line.

and $5.3 \times 10^{6}$ events acquired in total by the probe-and-ring system, of which $24 \%$ were probe events. In figure 6.8 , results are shown for simulations which include these realistic effects. Simulating the block effect was required in order to match the ring-only resolution as published in the scanner specifications ${ }^{1}$. The observed increase in resolution supplied by the probe rises to a maximum of over $55 \%$, for both previously studied projection profile directions, when degrading effects are added. Axial resolution, which by definition is parallel to

\footnotetext{
${ }^{1}$ activexray.com/pdf/Siemens B Biograph.pdf
} 


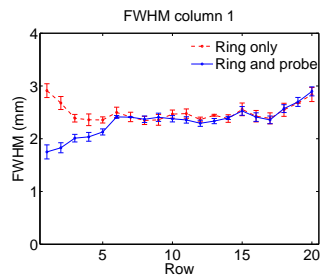

(a)

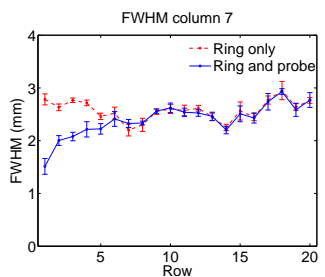

(d)

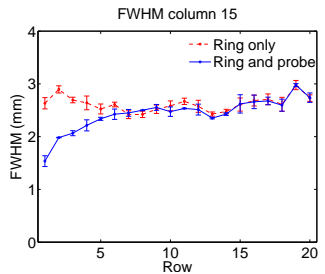

(g)

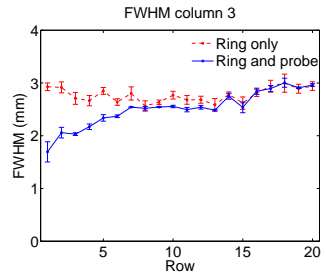

(b)

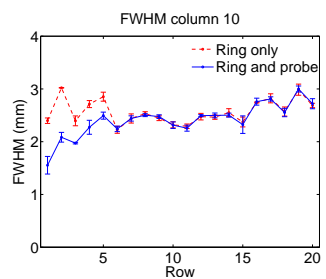

(e)

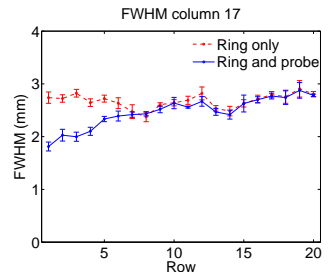

(h)

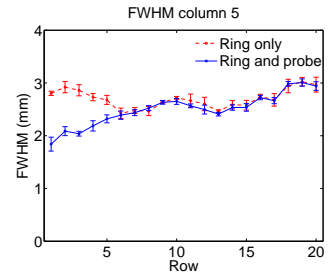

(c)

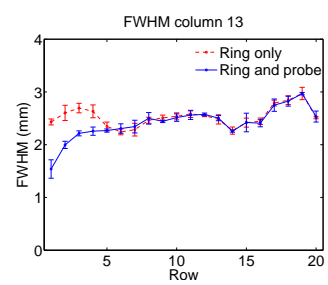

(f)

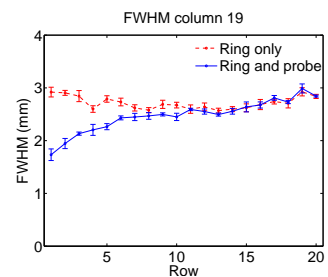

(i)

Figure 6.6: Comparison of FWHM, in the direction perpendicular to the probe surface, of the Biograph alone (red dashed line) and using the probe (blue solid line). Each graph presents the data corresponding to a specific column. Row and column numbers refer to the positions described in figure 6.1. The error bars indicate the standard deviation taken from 3 realizations.

the probe's surface, is observed to follow a similar trend to the other parallel resolution measurements, demonstrating $55 \%$ improvement. 


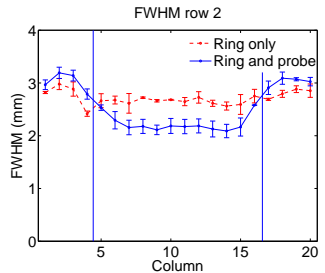

(a)

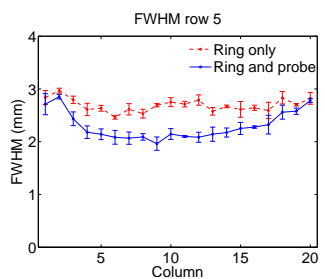

(d)

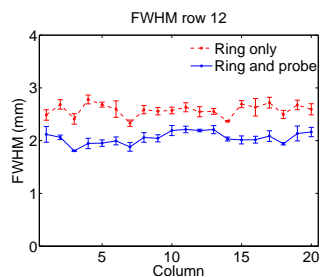

(g)

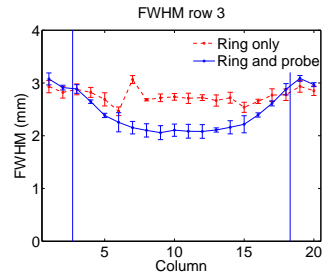

(b)

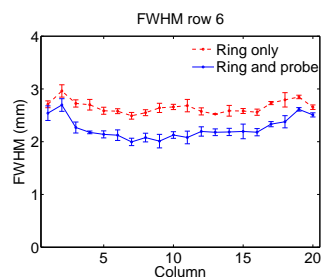

(e)

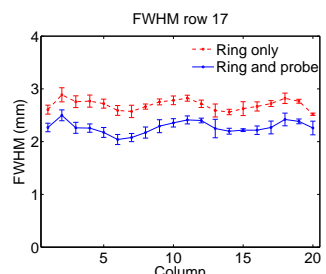

(h)

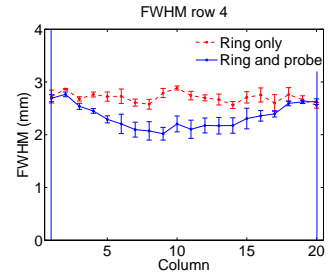

(c)

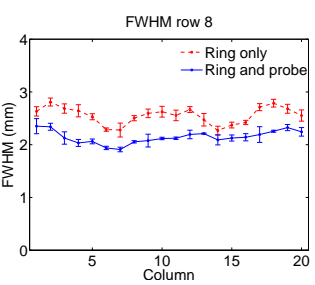

(f)

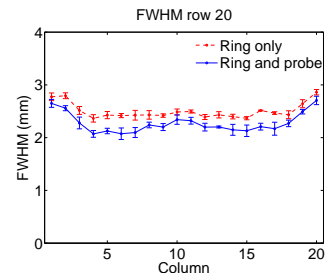

(i)

Figure 6.7: Comparison of FWHM, in the direction parallel to the probe surface, of the Biograph alone (red dashed line) and using the probe (blue solid line). Each graph presents the data corresponding to a specific row. Row and column numbers refer to the positions described in figure 6.1. The error bars indicate the standard deviation taken from 3 realizations. For reference, vertical lines in the plots for the first four rows show the locations corresponding to the $30^{\circ}$ cuts.

\subsubsection{Resolution Phantom Study}

Images of the Derenzo-like phantom in configuration A are shown in figure 6.9 , for the scanner-only and full probe system simulations. The scanner-only simulation contained $1.4 \times 10^{7}$ coincidence events and 


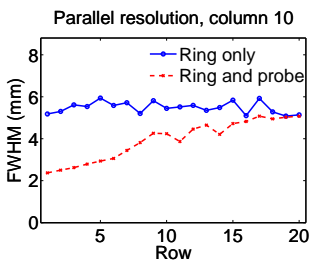

(a)

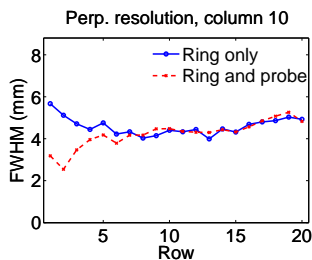

(b)

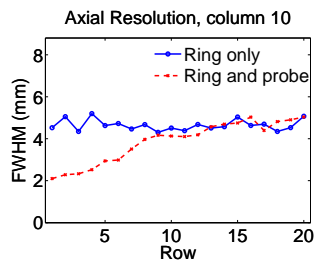

(c)

Figure 6.8: Comparison of resolution from the simulated single column of point-sources, including positron range, acollinearity, randoms, object and detector scatter as well as the block effect. Data for the Biograph alone is represented by the blue solid line, while that of the probe system is represented by the red dashed line. Transaxial resolution is shown in the direction (a) perpendicular and (b) parallel to the probe surface. Axial resolution is shown in c). Column and row numbers refer to the positions described in figure 6.1.

that which included the probe contained $1.7 \times 10^{7}$ events, of which $30 \%$ were probe events. The spatial resolution is visibly enhanced when including the high-precision probe data, most notably in the $2.4 \mathrm{~mm}$ insert region.

Line profiles over the $2.4 \mathrm{~mm}$ inserts, for the phantom in configuration $\mathrm{A}$, are shown in figure 6.10. All profiles demonstrate improvement to some degree, as predicted by our observations of the PSF. The phantom is located in the region where the PSF demonstrated enhanced resolution in directions both parallel and perpendicular to the probe surface.

Placing the probe closer to the 2.4 -mm rods (configuration B, figure $6.2 \mathrm{~b}$ ) further demonstrate this enhancement. The simulation of this orientation contained $1.4 \times 10^{7}$ coincidence events for the scanner alone and $1.7 \times 10^{7}$ for the probe system, of which $30 \%$ were probe events. Reconstructed images are shown in figure 6.11.

Line profiles through reconstructed images of the phantom in configuration $\mathrm{B}$ (figure 6.12) again show that the addition of the probe 
6.4. DISCUSSION

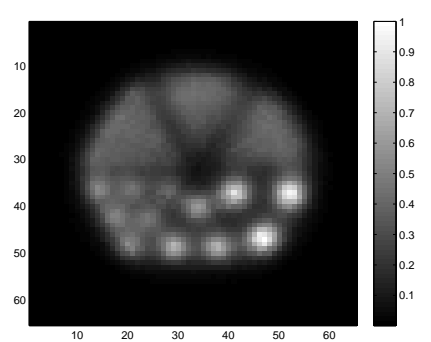

(a)

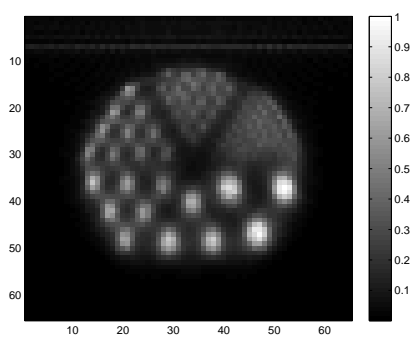

(b)

Figure 6.9: Images of the Derenzo-like phantom in configuration A, reconstructed using a) the scanner alone and b) including the probe data. The probe is located at the top of the image, in close proximity to the phantom as shown in figure 6.2 a). Pixels are $1 \times 1 \times 1 \mathrm{~mm}^{3}$.

improves resolution in a specific region of interest. The most significant improvement is achieved in the direction parallel to the surface of the probe. The profiles shown in figure $6.12 \mathrm{c}$ ) can be compared with those of figure $6.10 \mathrm{c}$ ), which represent the same phantom features in configurations B and A respectively. In configuration B these features are more closely aligned with the probe surface and are positioned closer to it than in configuration A, resulting in improved visualization, as clearly demonstrated by the profiles. A similar observation can be made when comparing figure $6.12 \mathrm{~d}$ ) and figure $6.10 \mathrm{~d}$ ). In configuration $\mathrm{B}$ these three features are aligned with the probe surface and are positioned closer to it, resulting in improved peak-to-valley ratios.

\subsection{Discussion}

The addition of probe events to the conventional PET data increases the resolution of the system, even in the direction perpendicular to the probe surface, where improvement might be less expected (figure 


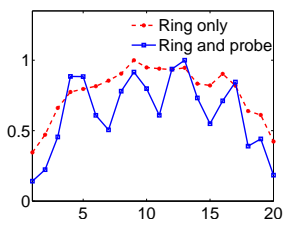

(a)

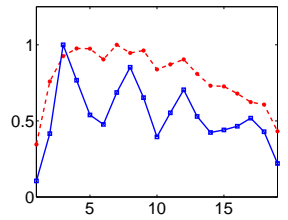

(d)

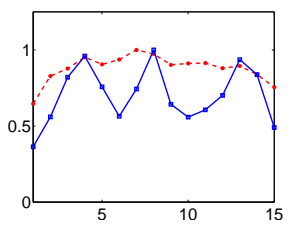

(b)

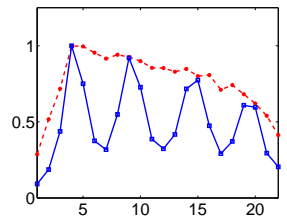

(e)

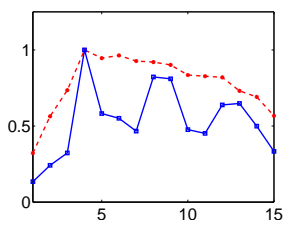

(c)

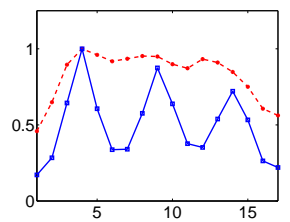

(f)

Figure 6.10: Line profiles through the Derenzo-like phantom, in configuration A, showing the improvement in spatial resolution achieved by including the probe data in image reconstruction. Profiles were taken in the region where this improvement was most visually evident. The locations of the corresponding line-profiles are shown in figure $6.2 \mathrm{a}$ ).

6.6). However, in the perpendicular direction, improvement was observed only in the first five rows of the simulated point-source array (1-5 cm), corresponding to positions close to the probe. In this region, the available probe projection angles offer high resolution in the direction under investigation. Resolution was observed to decrease with distance from the probe, as ring data begins to dominate, until reaching values comparable to those of the ring images. Resolution variation over the area defines a ROI were resolution enhancement can be expected, and which should be considered for probe placement. In this investigation we found this ROI to extend about $5 \mathrm{~cm}$ from the probe surface.

The profiles in figure 6.7 also show clear improvement to spatial 


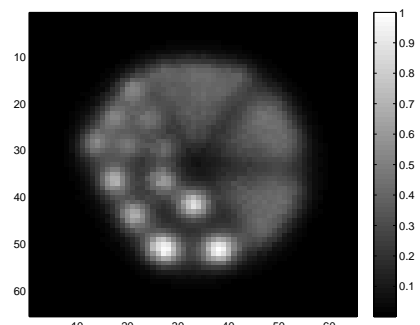

(a)

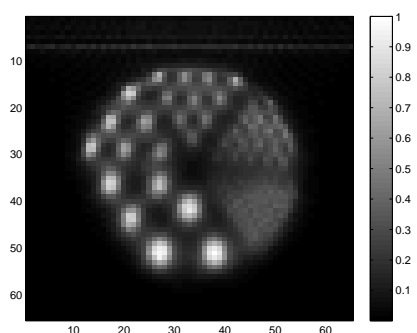

(b)

Figure 6.11: Images of the Derenzo-like phantom in configuration B, reconstructed using a) the scanner alone and b) including the probe data. The probe is located at the top of the image, in close proximity to the area of the phantom which contains the smallest features that are clearly resolved. This orientation of the phantom is shown in figure $6.2 \mathrm{~b}$ ). Pixels are $1 \times 1 \times 1 \mathrm{~mm}^{3}$.

resolution in the direction parallel to the probe surface, in certain areas of the FOV. The improvement is most evident in the central columns, where the probe LORs are perpendicular to the direction in which resolution is measured, and closer to the probe, where the fanshaped detection response has its narrowest section. The resolution is degraded at the top corners of the grid, where LORs are not optimally oriented to provide enhancement in the direction being investigated. For improved resolution in these regions, the probe would have to be repositioned, thus sacrificing resolution enhancement in other areas. Resolution improvement in the direction parallel to the probe surface is shown to extend out to the furthest rows, in agreement with Zhou and Qi (2009).

The increase in the abundance of probe events in the single-column simulation, from that observed in the full-grid simulation, may be explained by the exclusion of the outer columns of point sources which 


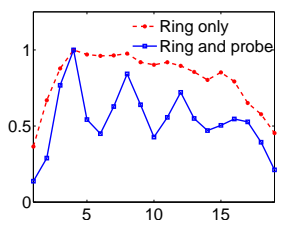

(a)

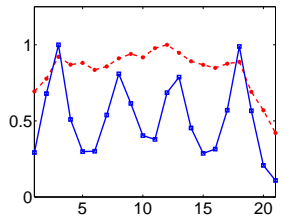

(d)

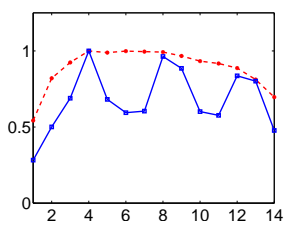

(b)

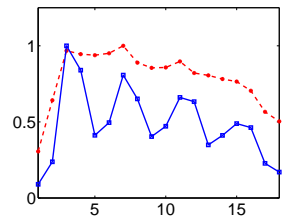

(e)

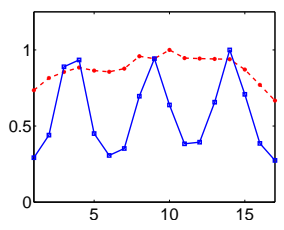

(c)

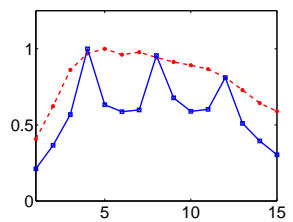

(f)

Figure 6.12: Line profiles through the Derenzo-like phantom in configuration $\mathrm{B}$, showing the improvement in spatial resolution achieved by including the probe data in image reconstruction. Data for the Biograph alone is represented by the red dashed line, while that of the probe system is represented by the blue solid line. The probe was placed next to the region where improvement was most visually evident (2.4 mm inserts). The locations of the corresponding line-profiles are shown in figure $6.2 \mathrm{~b}$ ).

are further away from the probe. Furthermore, the presence of randoms in the single-column data increases the count of probe events more than that of ring events, as the randoms fraction is expected to be greater in probe data. The inclusion of degrading effects to the simulated data degrades ring data more noticeably than probe data and thus increases the degree of improvement achieved using the probe.

Another contribution to the apparent broadening of the PSF in the top corners of the FOV was parallax due to the lack of depth of interaction (DOI) modeling in the system matrix for the ring detectors. DOI affects probe data differently than it does ring data, and as a result a spatial bias between the two measurement types was intro- 
duced. Combining the two data types in reconstruction has the effect of stretching the point-source image between two distinct locations, effectively broadening the PSF. This effect is demonstrated in figure 6.13 for a single sample point from the simulated point grid. The plot shows how the PSF of the combined probe-and-ring data is stretched between the two peaks of the ring-only and the probe PSF. Areas most affected by this loss of resolution are outside of the probe's FOV (as defined in section 5.2.1). Although the data were reconstructed without the application of the FOV reduction, the locations of the cuts are included in figure 6.7 for reference. The degradation outside of the $30^{\circ}$ region further motivates the removal of certain probe LORs from the reconstructed data, as mentioned in section 5.2.1. Besides patient placement, the magnitude of the parallax effect should be considered when choosing the cut angle, which could be adjusted according to the DOI model being used. A more accurate model in the ring crystals could increase the region where probe data contributes constructively to the image, allowing greater flexibility in its placement relative to the patient.

The measurements of FWHM from the realistic simulation, which include positron range, acollinearity and object scatter, support the observations extracted from the more simplified simulation of the points grid. The addition of the crystal decoding penalty in the detector blocks widens the gap between the FWHM of the two systems, especially in the area close to the probe. Resolution values for the scanner are similar to those given in the Biograph specifications, thereby supporting our model. The resolution of the combined system is less affected since the Si detector does not suffer from a block-like geometry. When including realistic effects in the simulation of a Derenzo-like phantom, the probe produces a clear improvement in image resolution. Enhancement is most evident in the region of the 


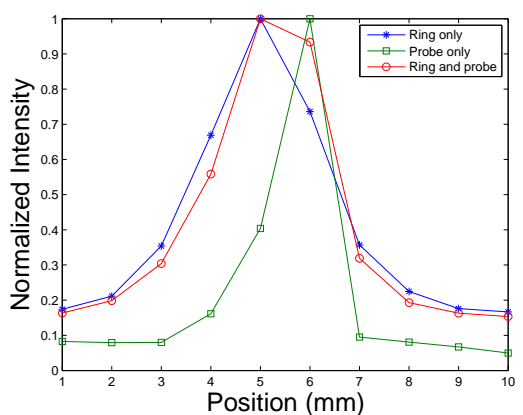

Figure 6.13: PSF (direction parallel to the probe) for the point in row 3 , column 4 measured from images reconstructed from the three data sets.

$2.4 \mathrm{~mm}$ inserts, where the inclusion of the probe reveals individual features, indistinguishable when using data from the scanner alone.

In the studies presented in this chapter, resolution was quantified as a function of position relative to the probe, with the device fixed at a single position. However, another factor affecting resolution is probe position relative to the ring. Moving the probe would alter the coincidence response of the probe LORs, thereby affecting the spatial variation of the system resolution. The study of such variation may be the subject of future work.

\subsection{Conclusions}

Studies comparing the spatial resolution of the probe-and-ring system with those of the PET scanner alone have shown the improvement provided by the probe in a limited region close to its surface. The extent of this region and of the expected resolution enhancement have been quantified. The greatest improvement in resolution, of values up 
to $55 \%$, was measured close to the probe's surface. While the resolution measured from images obtained by the scanner alone was fairly constant, at around $5 \mathrm{~mm}$ FWHM, values of just above $2 \mathrm{~mm}$ were observed in limited region when including the probe in simulations. The enhancement in the direction perpendicular to the surface of the probe was observed to extend up to $5 \mathrm{~cm}$ away from it, while in the parallel direction the enhancement extended further. Improvement was clearly demonstrated in an imaging study using a Derenzo-like phantom, where features which were indistinguishable to the scanner alone were revealed when the probe was incorporated. An imaging study analogous to that which used the Derenzo-like phantom has been performed using experimentally obtained data. The study, performed using an experimental prototype of the PET-probe system, can be found in the Experimental Supplement at the end of the document.

\section{References}

[1] Nuyts, J., Stute, S., Van Slambrouck, K., Van Velden, F., Boellaard, R., Comtat, C.: Maximum-likelihood reconstruction based on a modified poisson distribution to reduce bias in PET. In: IEEE Nucl. Sci. Symp. Med. Imaging Conf. (2011-oct. 2011) 4337-4341

[2] Zhou, J., Qi, J.: Theoretical analysis and simulation study of a high-resolution zoom-in PET system. Phys. Med. Biol. 54 (2009) 5193 


\section{Chapter 7}

\section{IMAGE QUALITY}

\subsection{Introduction}

The main aim of the PET probe is to increase the spatial resolution of a clinical scanner in a limited region of interest (ROI). However, it is expected that other measures of image quality will also be influenced by the presence of the Si detectors in the scanner's field of view (FOV). It is important to measure the extent of this influence as part of an analysis of the probe system. If any degradation in image quality is observed, then the trade-off between resolution enhancement and this degradation must be studied in order to asses the overall benefit of the PET probe system. If, on the other hand, it is found that other image quality measures are not sacrificed, then any improvement should be identified for potential exploitation.

The first study in this chapter focuses on investigating the probe's effect on the scanner's contrast and Spill-Over Ratio (SOR). By means of simulation, these Figures Of Merit (FOMs) are measured independently for the Biograph64 and the PET probe system. The study of the probe's effect on these FOMs is conducted over a wide FOV and 
is not only limited to the region in which resolution improvement is expected.

In the second study of this chapter, we demonstrate the probe system's applicability for PET mammography (PETM) by means of simulations which mimic a breast imaging scenario. A simplified breast phantom, adjacent to heart and torso compartments was simulated with the probe in close proximity to the breast.

In the context of breast imaging, we study the probe's contribution to lesion detectability through measurements of the local contrast recovery coefficient-to-noise ratio (CNR). In addition, we examine hotwarm contrast recovery and noise individually in the region where greatest improvement is expected. Although here we focus on breast imaging, the proposed system could be used in a similar way in any application where improved detectability is desired in a specific region of interest.

\subsection{SOR and Contrast Study}

Preliminary work has indicated that the addition of the probe to a clinical scanner has little effect on image quality at distances of the order of $10 \mathrm{~cm}$ from its surface [1]. The study was indicative of the negligible effect of the probe on certain image quality measures but did not investigate their spatial variation and was further limited by the exclusion of certain realistic effects from the simulations. In this study the previous work was revised, now incorporating more realistic simulations and extending the scope by investigating image quality at several positions over the FOV. The probe's impact on SOR and contrast is studied using a phantom with cold and hot regions at varying distances from the Si detector. 


\subsubsection{Contrast Phantom}

As in the case of image resolution, it is expected that other measures of the probe-and-ring system's image quality will also vary with position relative to the probe. As the number of probe-ring LORs passing through a single image pixel reduces with distance from the Si detector, it is expected that its effect on image quality will also decrease. For this reason, a dedicated phantom was designed with lesions located at several different distances from the probe. Since the objective of this study was to observe the effect of the probe on regular PET scanner performance, the lesion size was chosen as representative of features that could be imaged by a clinical scanner, as both a hot and cold lesion. For this reason, the chosen sphere diameter is one commonly used for image quality phantoms for clinical scanners [2]. The phantom used in this study consists of an $80 \times 144 \times 80 \mathrm{~mm}^{3}$ box at the center of the FOV, containing $1 \mathrm{mCi}$ of activity $(1.1 \mu \mathrm{Ci} / \mathrm{cc})$, which serves as a warm background for the SOR and contrast studies. Four empty (cold) spheres, with diameter of $16 \mathrm{~mm}$, are placed at distances of 27, 59, 91 and $123 \mathrm{~mm}$ from the probe surface, and offset by $16 \mathrm{~mm}$ relative to its centre. Equally offset, in the opposite direction but at the same distances from the probe, are hot spheres of $16 \mathrm{~mm}$ diameter, whose activity concentration is five times that of the background. Each hot sphere is separated from its corresponding cold feature by a distance of $32 \mathrm{~mm}$ (centre to centre), which is also the distance that separates successive hot-cold pairs. Figure 7.1 shows the phantom positioned relative to the probe.

Separate simulations were run using the PET ring alone and including the probe, each of a 10 second acquisition. Back-to-back photons were simulated in air in order to isolate the measurements from the effects of scatter and attenuation. Random events were included in the coincidence data but corrected for during image reconstruction. 


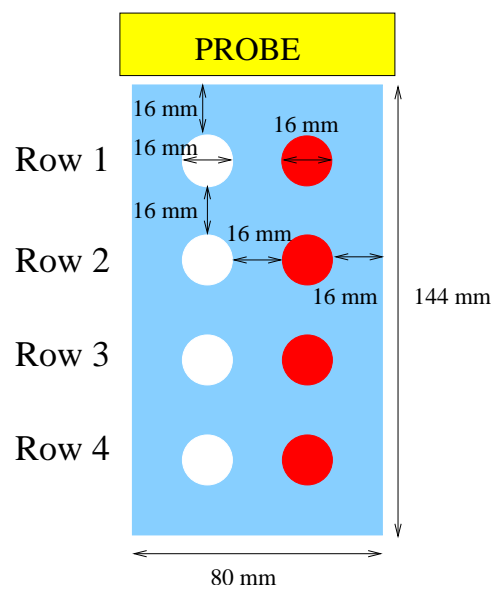

Figure 7.1: The phantom used for the image quality study and its position relative to the probe. The phantom is centered within the FOV. Rows 1, 2, 3, 4 correspond to distances of 27, 59, 91 and $123 \mathrm{~mm}$ respectively.

In order to observe the convergence of the various FOMs, 50 iterations were used.

\subsubsection{Figures of Merit}

Spherical regions of interest (ROIs) with diameter of $14 \mathrm{~mm}$ were chosen centered on each hot and cold spot. The ROI diameter was chosen to be smaller than that of the phantom features in order to avoid edge effects. For the warm ROIs, spheres of the same size were chosen exactly between each hot-cold pair. The SOR, hot-warm contrast $\left(C_{h o t / w a r m}\right)$ and warm-cold contrast $\left(C_{\text {warm } / \text { cold }}\right)$ were calculated at each iteration of the reconstruction up to 50, using the following definitions. 


\section{Spill-Over Ratio}

The SOR is a measure of the ability to image cold regions embedded in an area of higher activity. Cold-region imaging is a difficult task since, in reconstruction, counts from the surrounding active area tend to be misplaced, or "spill over", into the cold region. The SOR was calculated according to:

$$
S O R=\frac{\mu_{\text {cold }}}{\mu_{\text {warm }}} .
$$

Here, as in the definitions below, $\mu$ is the mean intensity of the corresponding ROI in the reconstructed images. The ideal value for the SOR is 0 .

\section{Hot-Warm Contrast}

Hot-warm contrast is a measure of the ability to image a hot area with the correct intensity relative to an adjacent less active area. It is defined by:

$$
C_{\text {hot } / \text { warm }}=\frac{\mu_{\text {hot }}-\mu_{\text {warm }}}{\mu_{\text {warm }}},
$$

The ideal value of $C_{\text {hot/warm }}$ for this phantom is 4 .

\section{Warm-Cold Contrast}

Warm-cold contrast is another measure of the ability to image a cold area. We define it by:

$$
C_{\text {warm } / \text { cold }}=\frac{\mu_{\text {warm }}-\mu_{\text {cold }}}{\mu_{\text {warm }}}
$$

The ideal value of $C_{\text {warm/cold }}$ for this phantom is 1 . 


\subsection{Breast Imaging Study}

A clinical application of the PET-probe system would be to imaging problems where higher resolution is desired in a localized region of interest (ROI), such as in the case of breast cancer imaging. PET has already been shown to be an accurate tool in mammography [3]. Its applications include the detection of the primary tumor, axillary staging, defining the extent of recurrent or metastatic breast cancer and monitoring response to therapy. Higher resolution images may be beneficial for a number of these tasks [3, 4]. The application of the PET-insert concept to breast imaging has already been investigated with encouraging results $[5,6]$. In this study, the application of the Si PET probe to breast imaging is examined in terms of its effect on lesion detectability.

\subsubsection{Breast Phantom}

A previously published computational breast phantom [7] was simulated adjacent to heart and torso compartments and with the probe in close proximity. The water-filled breast phantom consisted of a box with $\mathrm{x}, \mathrm{y}, \mathrm{z}$ dimensions of $12 \times 4 \times 15 \mathrm{~cm}^{3}$, adjacent to a waterfilled torso $\left(\mathrm{a} 20 \times 30 \times 30 \mathrm{~cm}^{3}\right.$ box $)$, both axially centered in the scanner. The heart was represented by a hot spherical source, 10 $\mathrm{cm}$ in diameter, centered in the scanner in the $\mathrm{y}$ and $\mathrm{z}$ dimensions and offset towards the breast by $3 \mathrm{~cm}$ in $\mathrm{x}$. Figure 7.2 a) shows an illustration of the simulation setup. The breast section of the phantom contained hot spheres at $2 \mathrm{~cm}$ depth, with diameters of 4, 5, 6, $7 \mathrm{~mm}$, representing breast lesions. The distribution of the spheres in the $\mathrm{x}-\mathrm{z}$ plane is shown in figure $7.2 \mathrm{~b}$ ). The probe is positioned so as to have the highest impact on imaging the four central lesions. Another four are located near the edge of the probe while the four 


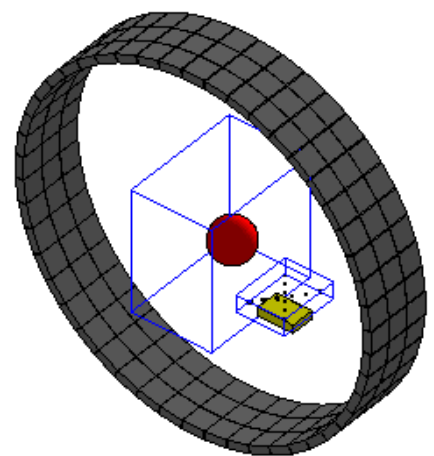

(a)

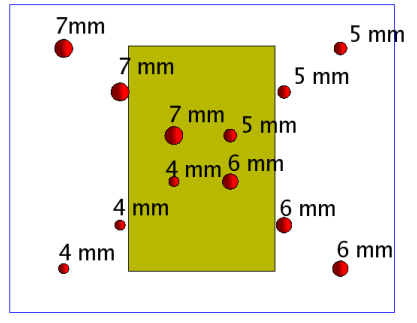

(b)

Figure 7.2: The breast-and-torso phantom shown to scale. The phantom consists of box-shaped torso and breast (both transparent in the figure), and a spherical heart, centred inside the scanner, with the probe underneath breast (a). A closer view of the breast shows the distribution of spherical sources and their position relative to the probe (b).

corner lesions are further away from it, in a region where the probe is expected to have little impact on their visualization. The hot spheres had a 9:1 uptake ratio with respect to the background, which had an activity concentration of $0.01 \mu \mathrm{Ci} / \mathrm{cc}$. The torso also contained 0.01 $\mu \mathrm{Ci} / \mathrm{cc}$ of activity and the heart $0.1 \mu \mathrm{Ci} / \mathrm{cc}$, according to the activity concentration ratio used by Peng and Levin [7]. A scan time of 17 minutes was simulated. Images were reconstructed using 20 iterations of the ML-EM algorithm, correcting for random and scattered coincidences. Phantom attenuation was also corrected for using the method described for probe attenuation correction. 


\subsubsection{Quantifying Lesion Detectability}

Lesion detectability is affected by lesion contrast and background noise. According to empirical studies, the performance of human observers in lesion detection is correlated with the ratio of contrast recovery to standard deviation of background noise [8]. Measurements of this ratio were performed on the lesions in the breast phantom to determine the advantage in detectability provided by the probe.

In addition, the impact of the probe on hot-warm contrast recovery was examined for the four lesions that are closest to its surface. This study expands on the contrast measurements presented in chapter 7 by including the effect of object scatter and by varying the diameter of the hot features to several values around the Biograph resolution. The effect of the probe on background noise was also measured in the central region of the breast, where the device was positioned.

For each lesion, a sphere was defined, centered on the simulated location of the lesion and with its corresponding radius. ROIs were chosen to contain all pixels that are completely contained in the defined spheres. A background ROI was chosen as a hollow sphere surrounding each lesion, with an outer radius of $14 \mathrm{~mm}$ and inner radius of $5 \mathrm{~mm}$. Mean values were calculated for all ROIs, as well as the variance for the background ROIs using:

$$
\operatorname{var}_{B}=\frac{1}{|B|-1} \sum_{i \in B}\left(\lambda_{B}(i)-\bar{\lambda}_{B}\right)^{2}
$$

where B represents the background ROI, $|B|$ denotes its size in voxels, $\lambda_{B}(i)$ the value in voxel $i$ and $\bar{\lambda}_{B}$, the mean voxel value. Using this definition, noise was calculated as $\sqrt{\operatorname{var}_{B}}$ and the contrast-to-noise ratio (CNR) was calculated as the ratio of contrast recovery coefficient to background standard deviation: 


$$
C N R=\frac{C R C}{\sqrt{\operatorname{var}_{B}}}
$$

Here, CRC is the contrast recovery coefficient defined as

$$
C R C=\frac{(\text { Contrast })_{\text {measured }}}{(\text { Contrast })_{\text {theory }}}=\frac{\bar{\lambda}_{H} / \bar{\lambda}_{B}-1}{9 / 1-1},
$$

where $\bar{\lambda}_{H}$ and $\bar{\lambda}_{B}$ denote the mean values in the hot lesion and background ROIs respectively.

\subsection{Results}

Comparisons of the various image quality metrics, taken from the contrast phantom and the breast phantom, are shown below. In all graphs, the solid line represents values from the probe system and the dashed line is for the ring-only system.

\subsubsection{SOR and Contrast Study}

The simulation of the contrast phantom using the PET ring alone produced $1.7 \times 10^{7}$ coincidence events. The simulation incorporating the probe accumulated $1.9 \times 10^{7}$ coincidences, $12 \%$ of which were probe-ring events. Plots of $C_{\text {hot } / \text { warm }}, C_{\text {warm/cold }}$ and SOR are shown as a function of iteration number, for the four different feature pairs.

\section{Spill-Over Ratio}

Figure 7.3 shows plots of the SOR. It can be seen that when using the probe there is a slight drop in the value in all rows, towards the ideal SOR. Improvement of about $10 \%$ is observed in rows 2-3. There is a slight rise of the SOR in row 1 when the probe is used. This, nevertheless, constitutes an improvement of $5 \%$ over the ring-only 

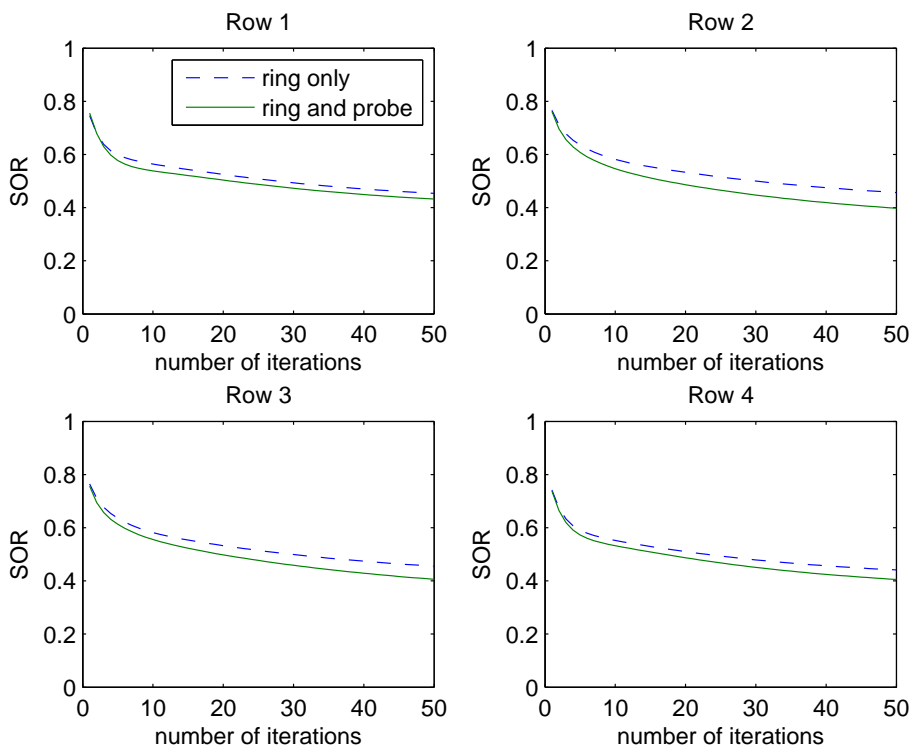

Figure 7.3: Plots showing SOR at different distances from the probe, vs. iteration number. Data from the ring-only images are shown using a dashed blue line and those including the probe are shown using a solid green line. The ideal value for SOR is 0 .

SOR. At 50 iterations the SOR values appear to be still decreasing, not having yet reached convergence.

\section{Hot-Warm Contrast}

Figure 7.4 shows plots of $C_{\text {hot } / \text { warm }}$. The plots indicate that the addition of the probe also improves this FOM. It can be seen that close to the probe there is considerable improvement, about $17 \%$ and in rows 2-3 there is a smaller but consistent improvement of about $7 \%$. Row 1 coincides with the region of the FOV where resolution enhancement was observed. The improvement in $C_{\text {hot/warm }}$ here may be due to local sensitivity enhancement in this region. 

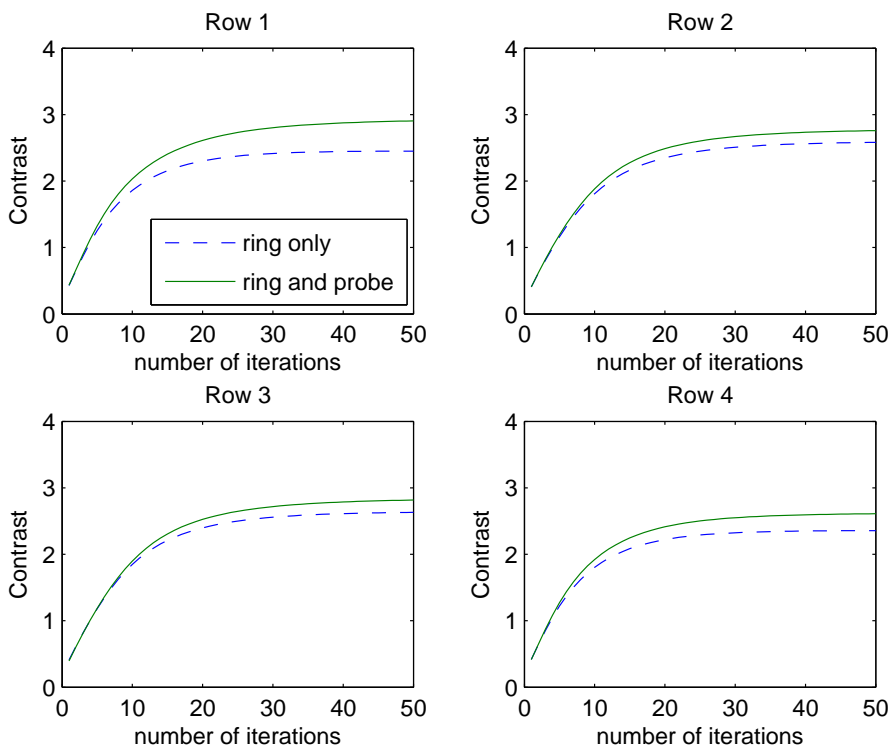

Figure 7.4: Plots showing $C_{\text {hot } / \text { warm }}$ at different distances from the probe, vs. iteration number. Data from the ring-only images are shown using a dashed blue line and those including the probe are shown using a solid green line. The ideal value for $C_{\text {hot } / \text { warm }}$ is 4 .

\section{Warm-Cold Contrast}

Figure 7.5 shows plots of $C_{\text {warm/cold }}$. The plots indicate a small improvement in this FOM when using the probe. The enhancement is similar to that observed in the SOR measurements. The behavior of $C_{\text {warm/cold }}$ reflects the tendency exhibited by the SOR: an improvement of about $5 \%$ near the probe and 10\% elsewhere. As was the case with SOR, $C_{\text {warm } / \text { cold }}$ appears not to have reached convergence at 50 iterations. 

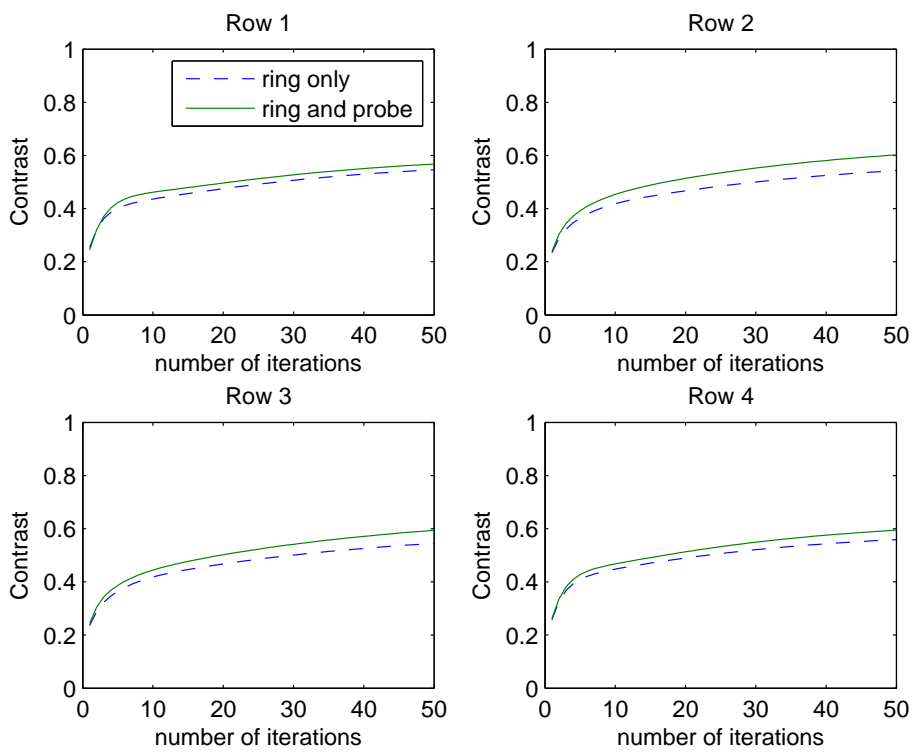

Figure 7.5: Plots showing $C_{\text {warm/cold }}$ at different distances from the probe, vs. iteration number. Data from the ring-only images are shown using a dashed blue line and those including the probe are shown using a solid green line. The ideal values for $C_{\text {warm/cold }}$ is 1 .

\subsubsection{Breast Imaging Study}

In this study $1.3 \times 10^{7}$ events were collected in the ring-only simulation. In the probe-and-ring simulation $1.4 \times 10^{7}$ events were collected, of which $1.3 \times 10^{5}$ were probe-ring events (just $1 \%$ of the total, since the probe is located far away from the main source of activity - the heart).

Images reconstructed from the breast-and-torso data are shown in figure 7.6. These show only the breast section of the phantom, as described by figure $7.2 \mathrm{~b}$ ). Figure $7.6 \mathrm{a}$ ) shows the image obtained from the simulation of the scanner alone and b) that of the probe-and-ring system. The images are normalized using a $20 \mathrm{~mm} \times$ 


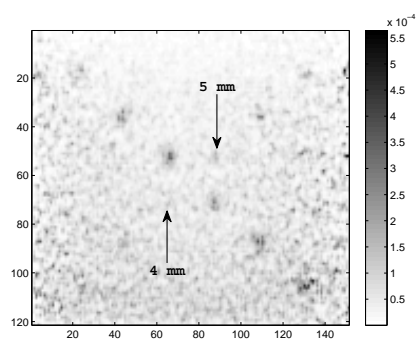

(a)

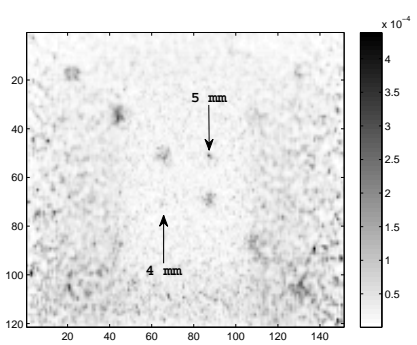

(b)

Figure 7.6: Images of the breast region of the breast-and-torso phantom a) without and b) including the probe. Pixels are $1 \times 1 \times 1 \mathrm{~mm}^{3}$. The point of view is looking down at the breast from the side opposite the probe.

$20 \mathrm{~mm}$ background patch in the breast. Despite the statistically low contribution of the probe-ring events, the improvement obtained by inclusion of the probe is visible in the central lesions, especially that with $5 \mathrm{~mm}$ diameter. A study of average intensity and noise values, for the four background ROIs of the corners and centre of the breast, reveals a $40 \%$ corner-to-centre noise decrease in the ring-only image. This value becomes $54 \%$ when the probe is used. The corresponding corner-to-centre difference in intensity is a $19 \%$ decrease for the ring-only image and a $54 \%$ decrease when using the probe.

$\mathrm{CRC}$ and noise measurements for the four central lesions are plotted in figure 7.7 as a function of lesion size. From figure 7.7 a) it is evident how contrast is increased with lesion size and that it is improved with the presence of the probe. An exception to the trend can be seen in the case of the $7 \mathrm{~mm}$ lesion which, in the case of the image which includes the probe, has a lower CRC, close to that of the ringonly image. This can be seen in figure 7.6, where the image appears to be affected by noise. Figure $7.7 \mathrm{~b}$ ) shows that noise is reduced in 


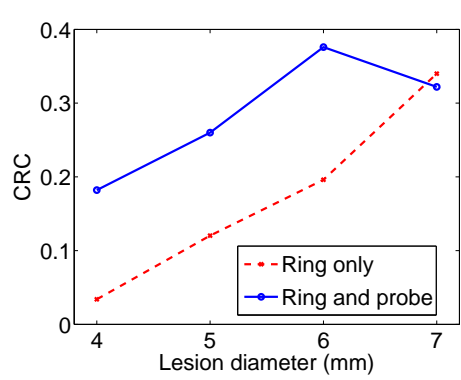

(a)

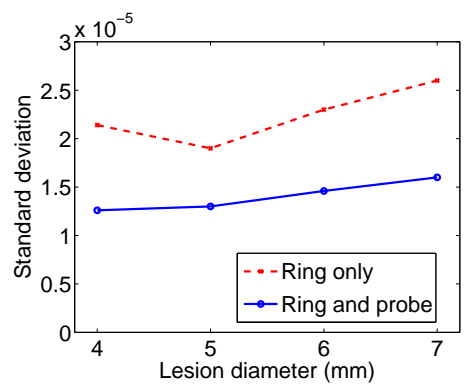

(b)

Figure 7.7: Plots of a) CRC and b) noise for the four central lesions.

the central background region due to the presence of the probe.

Figure 7.8 shows plots of CNR vs. position relative to the probe for the four lesion sizes. The dashed line representing the conventional scanner data is expected to be almost flat across the three distinct axial positions, rising slightly towards the center (position 3), where better sensitivity can be expected. It does so for the $6 \mathrm{~mm}$ and $7 \mathrm{~mm}$ lesions. Deviations from this trend for the $4 \mathrm{~mm}$ and $5 \mathrm{~mm}$ lesions can be expected since these are just at the limit of the scanner's resolving power. The addition of the probe increases the CNR values for the lesions in position 3, the location directly above the probe. This improvement is smaller for the $7 \mathrm{~mm}$ lesions which were already resolved by the scanner alone, and whose contrast in the probe image is affected by noise.

\subsection{Discussion}

The SOR and contrast studies show a clear enhancement in hot-warm contrast in the area close to the probe, in the same region where resolution was found to be increased. However, by choosing ROIs that are 

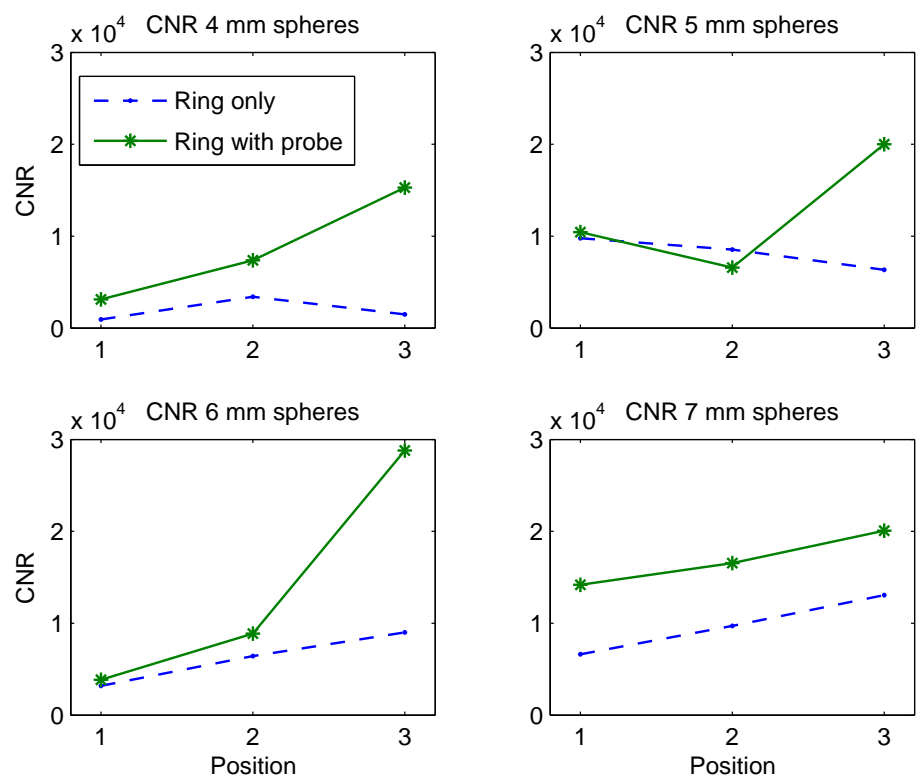

Figure 7.8: Plots of CNR calculated for lesions of different sizes and different locations relative to the probe. Position 1 refers to the lesion in the corner of the breast, furthest from the probe. Position 2 refers to the lesion near the edge of the probe. Position 3 is the location above the probe. (Refer to figure $7.2 \mathrm{~b}$.)

smaller than the phantom features, we have shown that the improvement in contrast provided by the probe is independent of resolution increase. The enhanced contrast is likely a result of increased sensitivity due to the probe, since this increase lowers background noise. However, enhancement in cold-warm contrast and SOR is limited. The plots suggest that these quantities had not converged at 50 iterations and that, in general, these FOMs are improving faster with iteration in the presence of the probe than in its absence.

An effect that contributes to added background counting rates, 
and thus reduces contrast, is scatter. The absence of scatter in the contrast phantom simulation limits the conclusions made about the effect of the probe on image quality. In order to address this issue, further image quality studies were performed which include a scattering medium in the simulated phantom. This was done using the breast imaging scenario.

Here, the increase in CNR provided by the probe in the central lesions is due to an increase in contrast and reduction in noise, as illustrated in figure 7.7. The region of the breast which is directly above the probe can be clearly identified visually in figure $7.6 \mathrm{~b}$ ) as an area of altered background. The noise in this area is reduced by the probe by about $1 / 3$ (according to figure $7.7 \mathrm{~b}$ ) compared to when the probe is absent, and by $54 \%$ compared to outer regions of the breast when the probe is in place. However, the reduction in noise which occurs in the region next to the probe, compared to outer regions, is accompanied by a $56 \%$ reduction in intensity. Such a reduction also occurs in the absence of the probe, but in that case is only of $20 \%$. The result of these effects is that the signal to noise ratio (SNR) in this region is not greatly affected by the probe. The reduction of intensity may be an artifact due to inaccuracies in the system model and is intensified by the presence of the probe.

\subsection{Conclusions}

The inclusion of the Si probe enhances the spatial resolution of reconstructed images. However, the presence of the Si detectors in the scanner's FOV is also expected to have an effect on other image quality measures. In order to investigate this effect, we measured the impact of the probe on image contrast and spill-over ratio. The studies show a clear enhancement in hot-warm contrast in the region of resolution 
enhancement. Further away, the addition of the probe was shown to offer reduced improvement of this figure of merit, but in no case was found to jeopardize the contrast obtained by the scanner alone. It was also shown that the probe did not degrade cold-spot imaging, as measured by SOR and contrast, but rather, again, offered limited improvement. This improvement is reduced in the area close to the probe, possibly due to an inadequate correction for random probe coincidences in this region. For a full assessment of the impact of the probe on image quality, other (non-sensitive) hardware components of the probe should be simulated, as these would increase the contribution of scatter in both the probe-ring and ring-ring datasets. This study is left to future investigations. Furthermore, a phantom which provides a scatter medium should be simulated in order to study the effects of scatter on the image quality of the probe system. Such a study was performed using a breast imaging scenario.

To demonstrate a potential clinical application of the PET probe system, and investigate its effect on lesion detectability, a breast phantom was simulated containing lesions of different sizes at varying positions relative to the probe. Reconstructed images showed a small improvement in lesion detectability when using the high-resolution device. Upon visual inspection of the reconstructed images, visualization of the $5 \mathrm{~mm}$ lesion directly above the probe was improved. The results are indicative of how a phantom feature, invisible to the PET scanner in isolation, is brought to light with the addition of the probe. Quantitative results support the observation that detectability is improved. CNR measurements, which are indicative of lesion detectability, demonstrate that the use of the probe improves the detectability of small lesions, which may be particularly relevant for early detection. The increase in CRC is due to the simultaneous increase in contrast and lowering of noise. In addition to noise, the 
probe also lowers background intensity in the region where improved detectability is observed. However, it does so in a way that does not significantly alter the SNR in the region. This artifact should be the subject of further study.

\section{References}

[1] Brzezinski, K., Gillam, J., Oliver, J.F., Lacasta, C., Rafecas, M.: Multiple Resolution PET Data: Image Properties using the ListMode MLEM Reconstruction Algorithm. In: 11th International Meeting on Fully Three-Dimensional Image Reconstruction in Radiology and Nuclear Medicine. (2011) 282-285

[2] National Electrical Manufacturers Association (NEMA): Performance Measurements of Scintillation Cameras. NEMA Standards Publication No. NU2, Washington, D.C. (2001)

[3] Eubank, W., Mankoff, D.: Evolving role of positron emission tomography in breast cancer imaging. Semin. Nucl. Med. 35 (2005) 84

[4] Shkumat, N., et al.: Investigating the limit of detectability of a positron emission mammography device: A phantom study. Med. Phys. 38(9) (2011) 5176

[5] Tai, Y.C., Wu, H., Janecek, M.: Initial study of an asymmetric PET system dedicated to breast cancer imaging. IEEE Trans. Nucl. Sci. 53(1) (2006) 121-126

[6] Aswim J. Mathews, S.K., Wu, H., O'Sullivan, J.A., Tai, Y.C.: Improving PET imaging for breast cancer using virtual pinhole PET half-ring insert. Phys. Med. Biol. 58 (2013) 6407-6427 
[7] Peng, H., Levin, C.S.: Design study of a high-resolution breastdedicated PET system built from cadium zinc telluride detectors. Phys. Med. Biol. 55 (2010) 2761

[8] Qi, J., Leahy, R.H.: A theoretical study of the contrast recovery and variance of MAP reconstructions from PET data. IEEE Trans. Med. Imag. 18 (1999) 293 


\section{Part IV}

\section{EXPERIMENTAL SUPPLEMENT}




\section{Chapter 8}

\section{SILICON PET PROTOTYPES}

\subsection{Introduction}

In addition to the simulations presented in the main document, experimental prototypes utilizing the PET insert concept have also been studied. The prototypes, which utilize the ring insert geometry (described in section 2.3) as well as the probe geometry (described in section 2.4) were developed at the University of Michigan. The laboratory setup under its various implementations, together with data acquisition software, were developed by the CIMA collaboration which includes researchers from various institutions: the University of Valencia, the University of Michigan, the Jozef Stefan Institute, the Ohio State University and CERN. The work presented here, performed during various research stays, aims to present preliminary studies using these prototypes. The experimental data used is constrained to that taken during the stays and, as a result, to that obtained from the experiments run during those periods. Although the experimental 
systems differ from that used in the simulation studies, thereby limiting the possibility of direct comparison and validation, these extra studies have the added value of allowing for the testing of the PET insert concept using experimentally acquired data. Furthermore, reconstructing data from several insert prototype geometries provided an opportunity to apply the image reconstruction tools developed in this thesis to other multi-resolution PET systems, thus demonstrating the flexibility of the reconstruction method.

Contained in the experimental studies presented here is preliminary work useful for the development of data-processing and image reconstruction software that can be applied to future PET insert laboratory setups. Approaches to solving a range of problems such as detector response modeling, detector placement and sensitivity correction were developed using the prototype geometries.

The work includes studies performed using several variations of the ring insert geometry. In these prototypes, two Si detector modules were placed on either side of a rotating phantom, thereby obtaining the full range of projections. Two different ring-insert geometries are described: a collimated 2D setup for imaging one trans-axial slice and a fully $3 \mathrm{D}$ version. Additionally, two different types of $\mathrm{Si}$ detector modules were used as part of work which tested hardware for future prototype development. Preliminary imaging studies with several different phantoms are presented and the reconstruction process is outlined.

As an extension to the study of the PET probe system, images from such a geometry, reconstructed from experimentally acquired data, are presented. Limited probe-only data was acquired and reconstructed in a 3D probe geometry. Combined probe-and-ring images were obtained by isolating a subset of the $2 \mathrm{D}$ ring-insert data to achieve the equivalent, for practical purposes, of a 2D PET probe system. 
The 3D imaging study serves to demonstrate the highest possible resolution available in the probe data. The effect of adding such data to a conventional PET scan of a commonly-used resolution phantom is demonstrated in the $2 \mathrm{D}$ study. Using the Derenzo-like phantom, a comparison of an image obtained using the PET ring is compared with that obtained when probe data was included. In this sense, the resolution study from section 6.2.5 is approximated here using data from the experimental prototype.

\subsection{Materials \& Methods}

The full-ring geometries used in this work consisted of partial rings of detectors on either side of the phantom, which was rotated to obtain full angular coverage. Basic motion-control hardware was in place for this, controlled by software with which the user was able to specify the angle through which the phantom rotated and the angular step. To simulate a full ring, multiple rotations were used with variable step size. The development of these systems was conducted by various members of the CIMA collaboration $[1,2,3]$. Probe data was obtained by using a subset of rotation positions.

\subsubsection{Sources used}

Data was acquired using three source distributions: a group of three low-activity sodium-22 $\left({ }^{22} \mathrm{Na}\right)$ point-like sources inside a plastic disk, a Derenzo-like hot-spot resolution phantom, similar to the one used for the resolution studies in chapter 6, filled with low-activity FDG18 solution, and small glass vile, with $1 \mathrm{~cm}$ inner diameter, filled to 1 $\mathrm{cm}$ with the FDG-18 solution. Schematic diagrams of the ${ }^{22} \mathrm{Na}$ and Derenzo-like phantoms are shown in figure 8.1. 


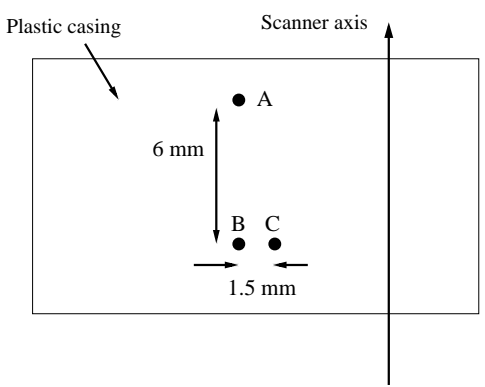

(a)

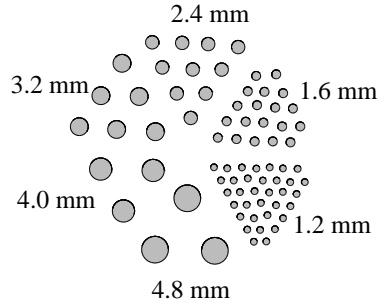

(b)

Figure 8.1: Two of the phantoms used in the imaging experiments: a) the group of three ${ }^{22} \mathrm{Na}$ point-like sources and b) Derenzo-like resolution phantom.

\subsubsection{The PET Scanner}

The PET scanner consisted of two partial rings using 24 block detectors from a decommissioned CTI 931 PET scanner. The blocks were organized into two groups of 12 , directly opposite one another at a distance of $1000 \mathrm{~mm}$, with each spanning 67.5 degrees (out of 180). To emulate the complete ring, the object being imaged was rotated to several positions. Each block consisted of 32 BGO crystals, each with dimensions $6 \times 12 \times 30 \mathrm{~mm}$ thick, in an $8 \times 4$ array ( 8 circumferential and 4 axial). The external PET ring was used for all geometries studied. The setup is shown in figure 8.2.

\subsubsection{The Ring Insert Geometry}

Two types of Si detectors were used for the experimental systems. The first generation of detectors consisted of $32 \times 16$ arrays of $1.4 \times 1.4$ $\mathrm{mm}$ pixels, the second consisted of $40 \times 26$ arrays of $1.0 \times 1.0 \mathrm{~mm}$ pixels. Both detector types were $1 \mathrm{~mm}$ thick. They were organized into modules made up of face-to-face detector-pairs, separated by a $0.8 \mathrm{~mm}$ gap. 


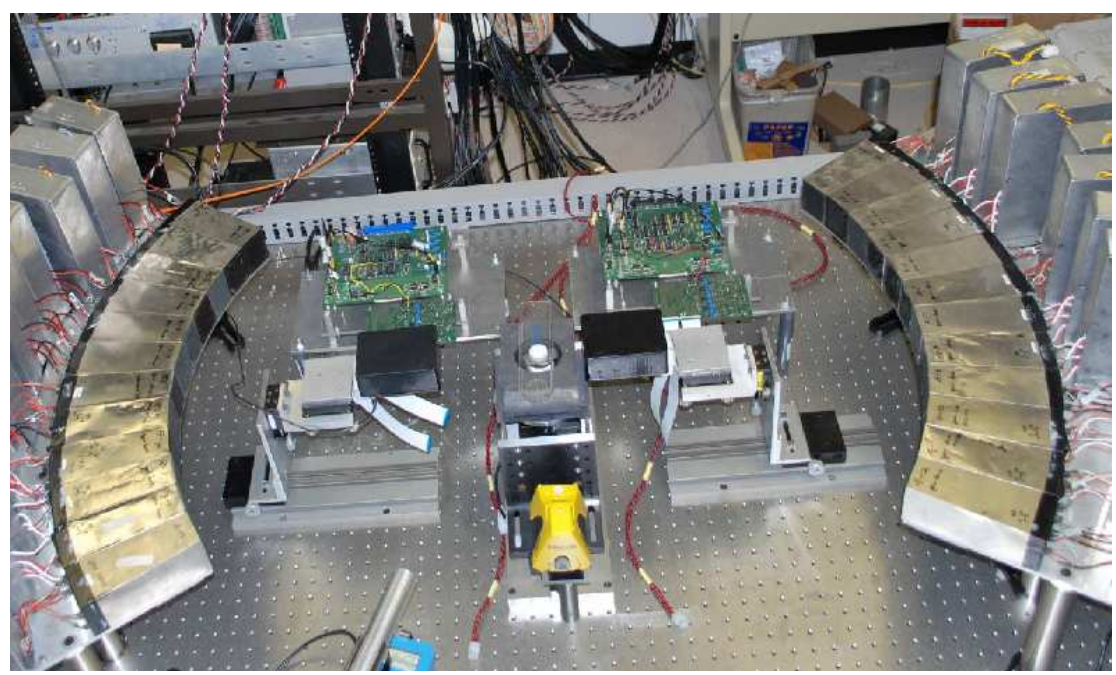

Figure 8.2: Photograph of the experimental setup. Visible in the centre is the lead shielding surrounding the phantom, with two black boxes on either side which house the Si detectors, and, further away, the two partial-rings of BGO block detectors.

2D Silicon Ring Setup. Two-dimensional setups were studied using both generations of Si detector modules. These were placed on opposite sides of the centre of the FOV, with the long edge of the detectors facing the centrally located phantom. The first-generation modules were offset by $97 \mathrm{~mm}$. Due to their more complex electronics, the second-generation modules were located at a further offset of $108 \mathrm{~mm}$. Lead shielding was placed around the phantom, leaving only a $1 \mathrm{~mm}$ slit through which annihilation photons could escape. The collimation was aligned with the edge of one detector from each Si pair and resulted in only one axial row of BGO crystals being involved in detection. A schematic diagram illustrating the collimated setup, using the second generation Si detectors, is shown in figure 8.3. The lead shielding around the phantom, with the black plastic boxes 
containing the Si detectors on either side of it, can be seen in figure 8.2 .

We consider four types of events:

- BGO-BGO. Each photon is detected in opposing BGO lobes.

- Si-BGO. One photon is detected in a BGO lobe and the other in the opposing $\mathrm{Si}$ detector.

- BGO-Si. Like above, but involving the other Si-BGO pair.

- Si-Si. Each photon is detected in opposing Si detectors. These events are specific to the ring insert geometry and were not present in the simulation studies.

Images were reconstructed, using the Derenzo-like phantom and 20 iterations of ML-EM, separately from the four types of events and also by combining Si-BGO and BGO-Si.

$3 D$ Silicon Ring Setup. To achieve 3D images, the collimation was removed and the $32 \times 16$-pixel Si detectors flipped to face the object being imaged. In this configuration, both detectors in each module face the phantom and all axial BGO detector-rows participate in measurement. A schematic diagram illustrating the 3D setup is shown in figure 8.4.

Images were reconstructed, using the ${ }^{22} \mathrm{Na}$ point-source phantom and the vile of FDG, with 20 iterations of the ML-EM algorithm.

\subsubsection{The Probe Geometry}

The two and three dimensional probe geometries were based on the setups used for the ring-insert studies. For the 3D case, one of the Si detector modules was removed and the phantom was not rotated, collecting Si-BGO events for one angular position. For the 2D geometry, probe-type events were isolated from files containing data from 
8.2. MATERIALS \& METHODS

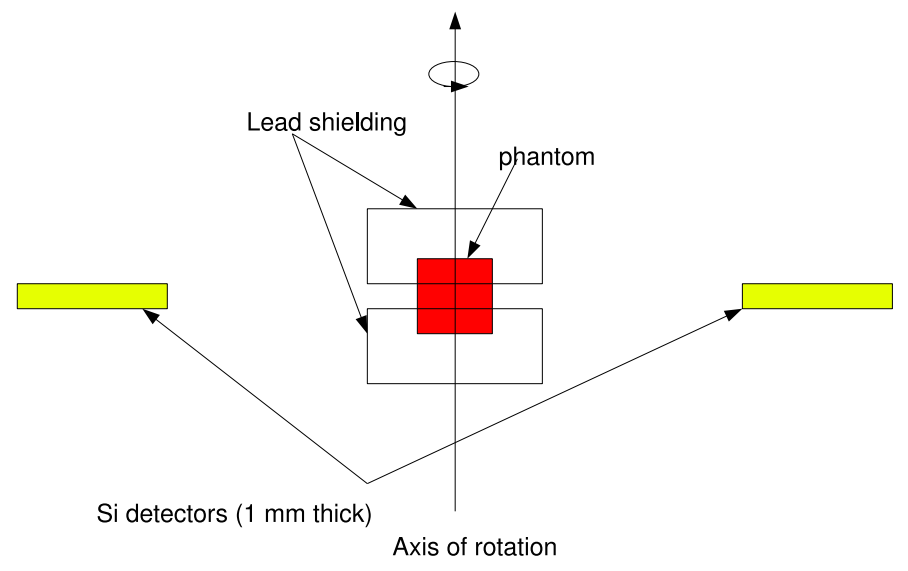

(a)

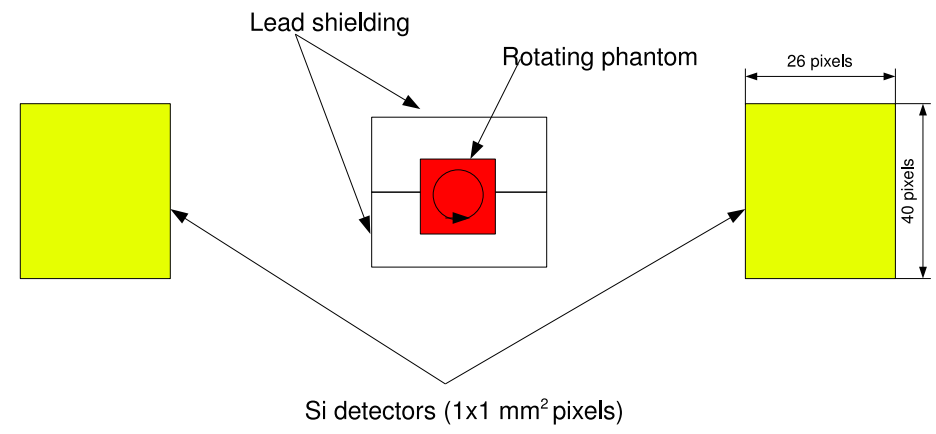

(b)

Figure 8.3: Diagram of the 2D ring insert geometry showing a) the side view and b) the top view. The drawing is not to scale, the centres of the Si detectors are both $108 \mathrm{~mm}$ away from the centre of the FOV. Si detector dimensions correspond to the second generation modules. The phantom rotating through 360 degrees simulates a Si ring and can obtain complete tomographic data. 
190

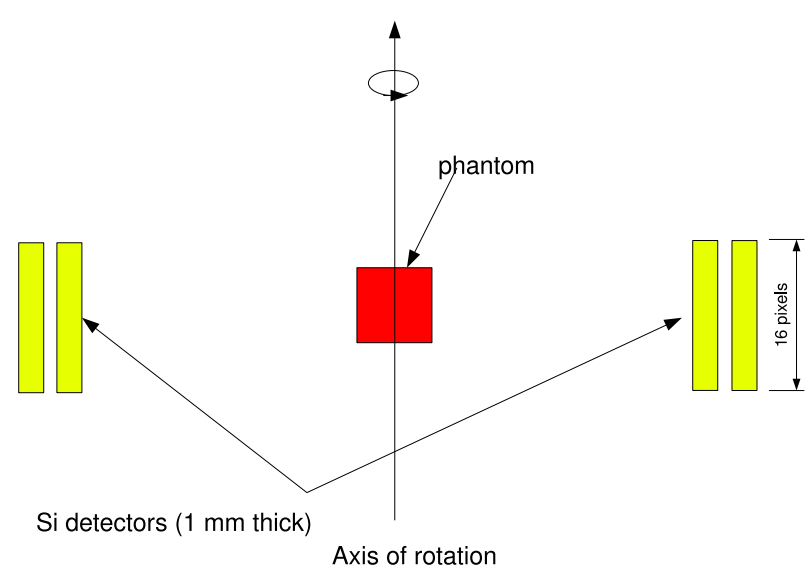

(a)

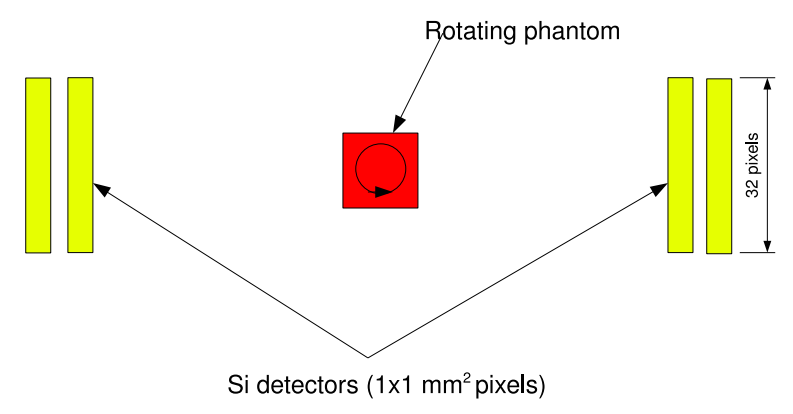

(b)

Figure 8.4: Diagram of the 3D ring insert geometry showing a) the side view and b) the top view. The drawing is not to scale. Si detector dimensions correspond to the first generation modules.

Si-BGO acquisitions. Complementary BGO-BGO acquisitions were also available for this geometry. 
$3 D$ Probe Setup. In order to look at the highest resolution achievable with this geometry, the ${ }^{22} \mathrm{Na}$ point-source phantom was placed in an area close to the probe. The phantom was taped to the probe's plastic casing, about 1.5 and $2.5 \mathrm{~cm}$ away from each of the two $\mathrm{Si}$ detectors. The sources were oriented so that the $1.5 \mathrm{~mm}$ separation between points $\mathrm{B}$ and $\mathrm{C}$ was approximately aligned parallel to the detectors' surface. Figure 8.5 shows a schematic diagram of the orientation of the point-like sources relative to the probe. Summed projections onto three orthogonal planes are shown in the figure for reference during analysis. Only Si-BGO data was obtained due to constrictions in the schedule. Images were reconstructed from the probe coincidence events.

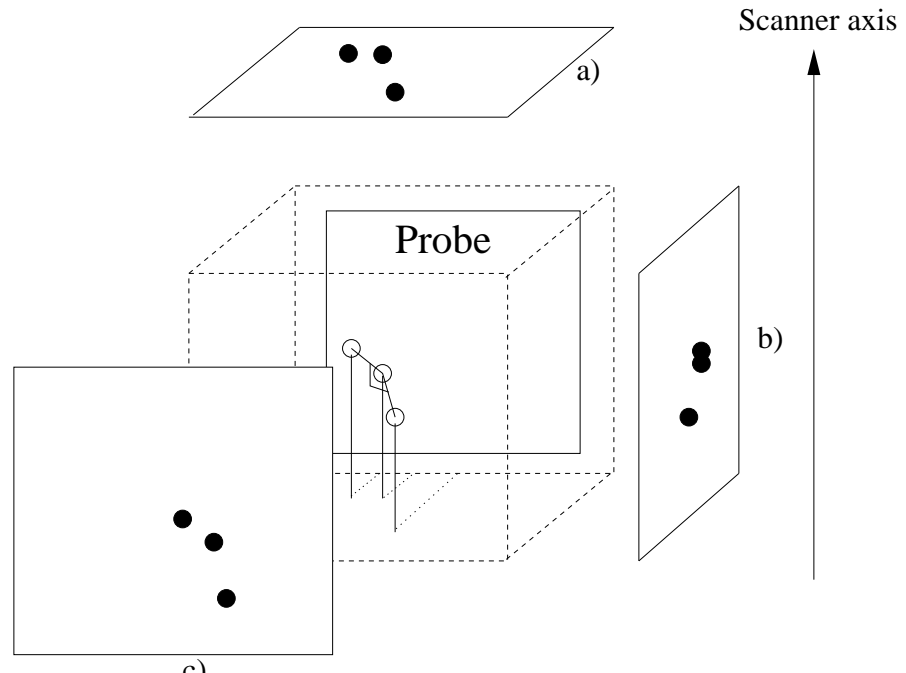

c)

Figure 8.5: Schematic diagram of the orientation on the Na point-like sources relative to the probe location.

Resolution Phantom in 2D. In order to obtain probe data from an acquisition dedicated to the ring-insert system, those Si-BGO events 
corresponding to two specific rotational phantom positions were isolated from the full-rotation data. The two positions corresponded to those where the $4.8 \mathrm{~mm}$ phantom features were at their closest to each of the two Si detectors. Using this method, events from both sets of Si-BGO pairs were combined to represent probe data, since both $\mathrm{Si}$ modules were at the same location relative to the phantom at these rotational positions. $1.3 \times 10^{5}$ such events were collected. In a separate acquisition, $8 \times 10^{5} \mathrm{BGO}-\mathrm{BGO}$ events were obtained to represent the ring-ring events, so that the probe events made up $15 \%$ of the total. The simulation studies using the Derenzo-like phantom gathered $30 \%$ probe events, but in the experimental study the phantom was further from the Si detectors so half that number was considered an appropriate estimate. For the ring-only image, $9 \times 10^{5}$ BGO-BGO events were used in order to compare images with the same number of events, eliminating sensitivity as an influencing factor.

Images were reconstructed using 20 iterations of ML-EM from the ring-only and ring-and-probe data, according to the methods described in section 8.2.5. Line profiles were taken through the sections of the phantom where individual features were distinguishable in the ring-only image. These include the three sections with the largest features, of diameter $4.8 \mathrm{~mm}, 4.0 \mathrm{~mm}$ and $3,2 \mathrm{~mm}$. Figure 8.6 shows a diagram of the phantom with the labeled profile locations. Also included is the relative position of the probe, which is not shown to scale.

\subsubsection{Reconstructing the Data}

The rotation of the phantom to many possible positions relative to the detectors made histograming the data and pre-calculating the system matrix non-trivial. The list-mode ML-EM algorithm and the multiline Siddon method for on-the-fly calculation of the system matrix 


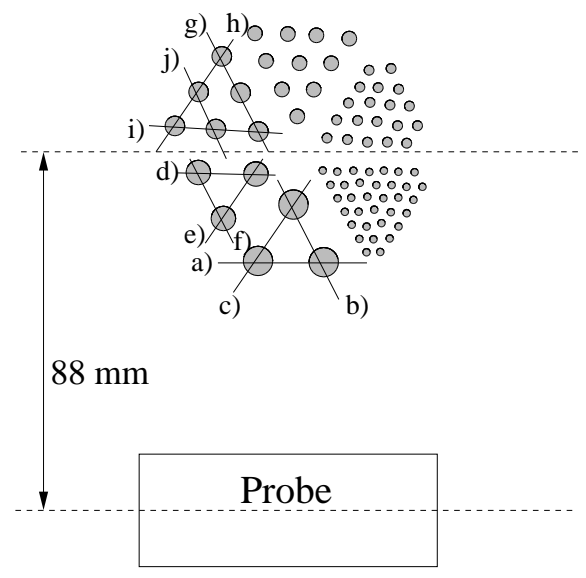

Figure 8.6: Derenzo-like phantom in the orientation relative to the $\mathrm{Si}$ detectors used in the study. Probe size and distance to the phantom are not to scale. Profile locations are shown for reference.

elements was especially useful for image reconstruction using this experimental setup. The end-points of the Siddon lines were randomly distributed throughout the BGO crystal and randomly at the halfdepth plane for the Si pixels, using three and two dimensional uniform distributions respectively. This method was used to take advantage of data processing software which was already in place at the University of Michigan and which calculated several coordinates throughout the detector for each event. However, attenuation in the BGO was not accounted for in lines originating from a finite depth within the detector crystal. A fine image grid of $0.2 \times 0.2 \times 0.2 \mathrm{~mm}^{3}$ voxels was used 
for the reconstructions in order to resolve the finest phantom features, which were within the expected resolution capabilities of the highest resolution data ( $\mathrm{Si}-\mathrm{Si}$ events). The resulting increase in image pixel density, by a factor of 125 from the $1 \times 1 \times 1 \mathrm{~mm}^{3}$ image grid used in the simulation studies, would indicate that a increase in Siddon line count is required. Furthermore, larger scintillation crystals in the scanner also motivate such an increase. As an initial estimate, 100 Siddon lines per measurement were implemented and used for both $2 \mathrm{D}$ and $3 \mathrm{D}$ geometries, both generations of Si detectors, as well as both ring insert and probe setups. Corrections for randoms, scatter, probe attenuation and detector efficiency were not implemented.

All final images involving BGO-BGO events were convolved with a Gaussian function of $0.4 \mathrm{~mm}$ FWHM to reduce undersampling artifacts created by reconstructing the low-resolution data on the fine $0.2 \times 0.2 \times 0.2 \mathrm{~mm}^{3}$ voxels. This was considered a more efficient approach of overcoming the artifacts than increasing Siddon-ray count.

Sensitivity Corrections. Initial reconstructions were performed with a uniform sensitivity estimate. The lack of a correction had the greatest impact on images reconstructed from Si-Si events, due to the system's fast-moving sensitivity response to these events. Si-Si sensitivity was calculated by backprojecting one count per detector pair, using one Siddon line with endpoints at the centre of each detector. The result was then blurred with a Gaussian of $0.4 \mathrm{~mm}$ FWHM in order to smooth sampling artifacts. Since the sensitivity calculated in this way does not correspond to the system matrix, which uses hundredfold random over-sampling of the detector response, it was not used to normalize the system matrix, thus avoiding possible artifacts created by a system matrix-sensitivity mismatch. Rather, images were reconstructed using a sensitivity-corrected version of the ML-EM algorithm: 


$$
f_{j}^{k+1}=\frac{f_{j}^{k}}{S_{j}} \sum_{l=1}^{N} a_{i j} \frac{1}{\sum_{b=1}^{J} a_{i b} f_{b}^{k}},
$$

where $S_{j}$ is the sensitivity and the system matrix elements, $a_{i j}$, are proportional to the probability that a detection due to an event in pixel $j$ is detected in LOR $i$, now without the condition that it be detected by the system.

The sensitivity matrix was obtained for the Si-Si only, as problems in correctly matching BGO crystal positions with those calculated using pre-existing software persisted until the end of the investigation.

\subsection{Results \& Discussion}

Reconstructed images which use data obtained from the prototype systems are presented below. With the exception of the $2 \mathrm{D}$ study of the probe system these are only preliminary images, with the various event types reconstructed separately. Joint reconstructions, combining the multi-resolution datasets, require further work on obtaining the system sensitivity.

Despite the limited acquisitions dedicated to the PET probe system, the reconstructed images offer insight into the probe prototype and reproduce some qualitative results from the simulation studies.

\subsubsection{D Ring Insert Geometry}

Preliminary images using the Derenzo-like phantom, reconstructed using the 2D double-ring setup with the second generation of Si detectors, are shown below. We include a sample of the images obtained using BGO-BGO and Si-Si events separately. Si-BGO and combineddata images can be obtained in a similar fashion in future studies once sensitivity matrices are correctly calculated. 


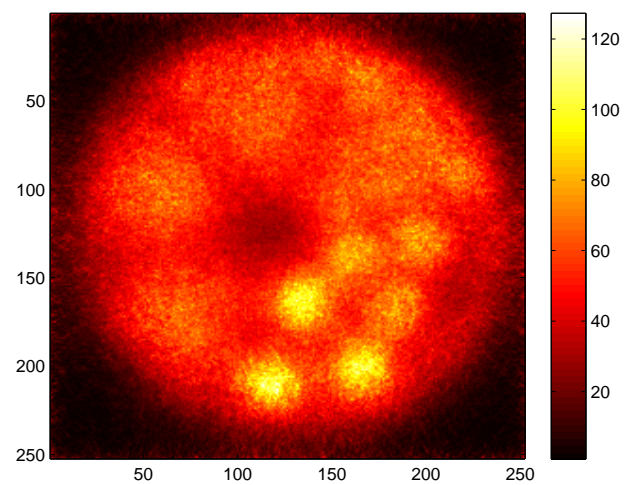

Figure 8.7: Resolution phantom reconstructed using BGO-BGO events only, using a uniform sensitivity and 20 iterations of the MLEM algorithm.

PET scanner images. Images were reconstructed from $9 \times 10^{5}$ BGO-BGO events without sensitivity correction in the $2 \mathrm{D}$ configuration. The images are not greatly affected by this lack of correction, suggesting a nearly flat BGO-BGO sensitivity in the reconstructed FOV of $51.2 \times 51.2 \times 51.2 \mathrm{~mm}^{3}$. This was to be expected, since for a scanner of $1 \mathrm{~m}$ diameter sensitivity will not vary significantly over such a relatively small distance. Figure 8.7 shows the image of the Derenzo-like phantom reconstructed using BGO-BGO events only.

Si-Si images. Using the Derenzo-like phantom in the 2D geometry, $8 \times 10^{6}$ Si-Si coincidence events were reconstructed. Figure 8.8 a) shows the image after 20 iterations of ML-EM, initially without any sensitivity correction (a uniform estimate). Sensitivity-related artifacts are evident, with high intensity at the centre and fading towards the edges of the FOV. Figure $8.8 \mathrm{~b}$ ) shows the $2 \mathrm{D}$ sensitivity estimate for $\mathrm{Si}-\mathrm{Si}$ events. The effect of applying the correction to the image is shown in figure $8.8 \mathrm{c}$ ). 


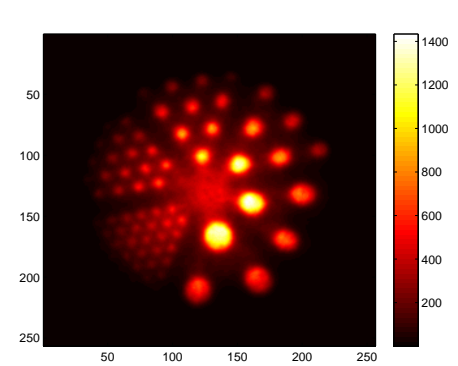

(a)

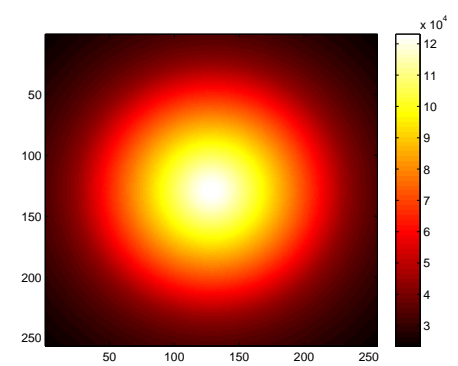

(b)

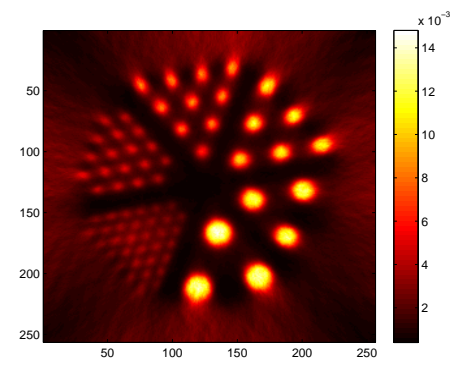

(c)

Figure 8.8: Si-Si images: a) Resolution phantom without sensitivity correction. b) Sensitivity estimate. c) Resolution phantom reconstructed including the sensitivity estimate.

The sensitivity-related artifacts present in figure 8.8 a) are reduced in figure $8.8 \mathrm{c}$ ). However, a new artifact is introduced. The "fraying" that can be seen around the phantom edges is due to data truncation. The phantom is slightly wider than the two Si detectors, which results in a limited number of projection angles in the outer regions of the FOV. This artifact should be removed by adding Si-BGO and BGOBGO data to the reconstructed image. 


\subsubsection{D Ring Insert Geometry}

For the 3D double-ring geometry, the ${ }^{22} \mathrm{Na}$ phantom was used to observe resolution, and the FDG-filled vile aided the assessment of uniformity. Data was acquired using the first generation Si detectors.

Point-Like Sources. Figure 8.9 show images of the ${ }^{22} \mathrm{Na}$ phantom reconstructed from three separate datasets: $\mathrm{Si}$-Si and the two opposing Si-BGO pairs. BGO-BGO data was not obtained for this setup due to constraints in the experimental schedule. The Si-Si images show very high resolution, demonstrating clearly the $1.5 \mathrm{~mm}$ separation between point sources B and C (see figure 8.1 a). The Si-BGO images are unable to distinguish between these two but clearly show the separation to point A, which lies $6 \mathrm{~mm}$ away.

Extended Source. Figure 8.10 shows images of the vile of diluted FDG, reconstructed using Si-Si and Si-BGO data separately, employing the second generation Si detectors. In all cases, artifacts are observed in the summed axial slices that appear to be related to axial sensitivity. However, attempts to reproduce these artifacts in the Si-Si sensitivity image, by using a narrower Gaussian in the blurring, were not successful.

\subsubsection{D Probe Geometry}

Figure 8.11 shows images from the 3D probe acquisition using the ${ }^{22} \mathrm{Na}$ point-source phantom. The three images were obtained by summing all slices in three orthogonal directions. The $1.5 \mathrm{~mm}$ separation between B and C appears slightly smaller due to the small inclination of the three-point system relative to the probe. Nevertheless, the two points are clearly differentiated in figure $8.11 \mathrm{a}$ ), which shows the summed transaxial slices, and c), representing the axial slices which are parallel to the probe surface. The images demonstrate a spatial 
resolution of under $1.5 \mathrm{~mm}$ at this position, about $1 \mathrm{~cm}$ away from the probe's surface. The two points are indistinguishable in figure 8.11 b) since they lay in different slices one behind the other and overlap when the slices are summed.

\subsubsection{Resolution Phantom: 2D Probe Geometry}

Images of the Derenzo-like phantom are displayed in figure 8.12, with a) showing the image reconstructed from BGO-BGO events only, and b) that which includes the probe data. An improvement in resolution is clearly visible, especially in the $3.2 \mathrm{~mm}$ features. The directionality of this improvement can also be seen, as predicted by simulation studies.

The improvement can be further observed by looking at line profiles through the sections of the Derenzo-like phantom (described in figure 8.6). Once again, the most significant improvement is observed in the direction parallel to the probe face, with all profiles taken in that direction showing significant increase in resolution. The profiles which are perpendicular to the probe, such as $g$ ), j) and f), do not show significant improvement. It should be noted that the increase in resolution is observed at distances of up to $80 \mathrm{~mm}$ from the probe, substantially further than observed using the simulated system. This can be explained by the lower resolution of the BGO detectors and, as a result, of the PET ring image which is enhanced by the probe data. The simulated LSO scanner already produced images of relatively high resolution on its own, which made it more challenging to enhance.

In the probe-only images of the point-sources, their apparent elongation is due to truncation. In an area so close to the probe only very limited projection angles are available. The images reflect only what the probe "sees", this means that the ability to distinguish features aligned perpendicular to its surface is lost. In the images of the 
Derenzo-like phantom, the directionality of the resolution enhancement makes the circular cross-section of some of the phantom features appear oblate, giving the impression of elongation. In fact, the features are not elongated by the probe but rather show improved resolution limited to the direction perpendicular to the probe LORs. That is to say, the circular features are shortened in one direction. This effect is made evident by the line profiles. The increased directional limitation of the resolution enhancement, compared to the simulation studies where this effect was not observed, may be caused by data truncation due to the larger distance to the probe and the shorter length of the probe detector facing the phantom. These two factors limit the number of projection angles available at the phantom location. Furthermore, the larger size of the BGO crystals, compared to those of the Biograph, produces a larger difference between probe and ring resolutions in the experimental system. This increases the impact of the probe on ring data.

\subsection{Conclusions}

Ring-insert and PET probe prototype systems have been studied, in $2 \mathrm{D}$ and $3 \mathrm{D}$ configurations. For the ring-insert geometry, two partial rings of $\mathrm{BGO}$ detectors and two $\mathrm{Si}$ detector modules on either side of a rotating phantom simulated the full PET ring and the Si ringinsert. The studies performed using $2 \mathrm{D}$ and $3 \mathrm{D}$ implementations of this setup consisted of preliminary work on the development of the prototype. It was found that coincidence events between two insert detectors (Si-Si events) were able to achieve image resolution of under $1.5 \mathrm{~mm}$. However, the area where this resolution was achievable was limited by data truncation outside a limited ROI.

A dedicated image reconstruction software was developed based on the one described in chapter 5 , but with some important modi- 
fications. Si-Si sensitivity was calculated using one Siddon line per LOR in order to reduce calculation time. The sensitivity image was then smoothed by convolution with a Gaussian function to remove undersampling artifacts. Si-BGO and BGO-BGO sensitivity calculation was left to future work. In the projection and back-projection operations, Siddon line-count for modeling detector response was increased from 16 to 100, due to higher voxel density. Due to the different system models used in calculating the sensitivity matrix and the system matrix elements, matrix normalization was not performed. Instead, a modified implementation of the ML-EM algorithm was used. Finally, the original reconstruction software was prepared for data from GATE simulations, so tools for processing the experimental data into a format usable by the software were developed during studies of the ring insert geometry.

Experimental data was also acquired for the PET probe geometry and images were reconstructed. Probe data was obtained from a dedicated 3D system, but no complementary ring data was available for these acquisitions. However, from images reconstructed from the probe data only, image resolution of under $1.5 \mathrm{~mm}$ was observed. For a comparison of the resolution of the PET scanner alone to that of a system which includes the probe, 2D probe data was isolated from an acquisition dedicated to the ring-insert geometry. Reconstructed images revealed a clear improvement achieved by adding the probe data. Resolution was increased and the directionality of this improvement was in accordance to the findings of simulation studies, which measured resolution variation across the FOV. However, unlike in studies using a simulated Derenzo-like phantom, increased truncation in the probe data and a larger difference between probe and ring resolutions created the impression of an elongation of the phantom features in the experimental system. Finally, corrections for randoms, scattered 
events and attenuation were not performed during image reconstruction. Implementing these corrections would improve the quality of all images, although further investigation extending these experiments would be required to determine whether improvement provided by the probe would be augmented.

\section{References}

[1] Studen, A., Brzezinski, K., Chesi, E., Cindro, V., Clinthorne, N., Grkovski, M., Grošičar, B., Honscheid, K., Huh, S., Kagan, H., Lacasta, C., Llosá, G., Linhart, V., Mikuž, M., Stankova, V., Weilhammer, P., Žontar, D.: Narrowing coincidence timing window through time-walk correction in silicon ring enhanced PET scanner. In: Nuclear Science Symposium and Medical Imaging Conference (NSS/MIC), 2011 IEEE. (Oct 2011) 266-270

[2] Clinthorne, N.H., Brzezinski, K., Chesi, E., Cochran, E., Grošičar, B., Honscheid, K., Huh, S., Kagan, H., Lacasta, C., Linhart, V., Mikuž, M., Stankova, V., Studen, A., Weilhammer, P., Žontar, D.: Silicon as an unconventional detector in position emission tomography. Nucl. Instrum. Methods A 699 (2013) 216-220

[3] Studen, A., Brzezinski, K., Chesi, E., Cindro, V., Clinthorne, N.H., Cochran, E., Grošičar, B., Grkovski, M., Honscheid, K., Kagan, H., Lacasta, C., Llosá, G., Mikuž, M., Stankova, V., Weilhammer, P., Žontar, D.: Silicon detectors for combined MRPET and MRSPECT imaging. Nucl. Inst. Meth. Phys. Res. Sec. A 702 (2013) $88-90$ 


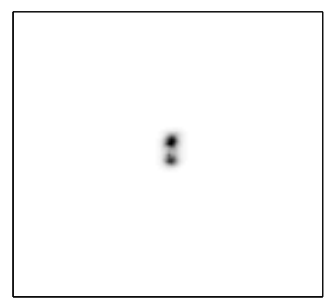

(a)

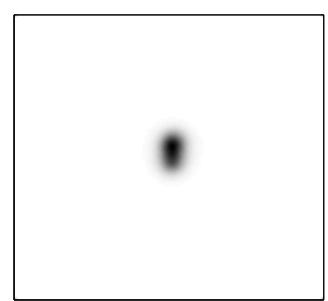

(d)

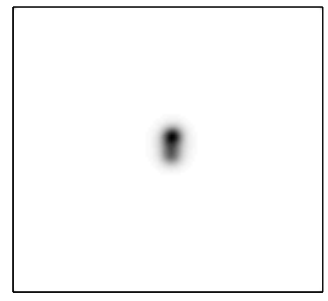

(g)

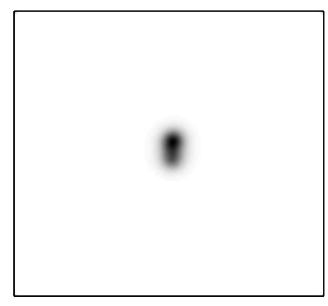

(j)

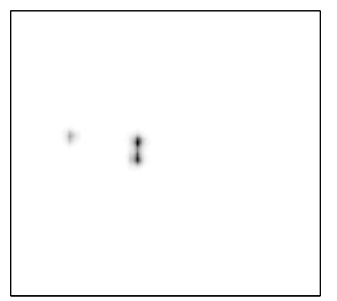

(b)

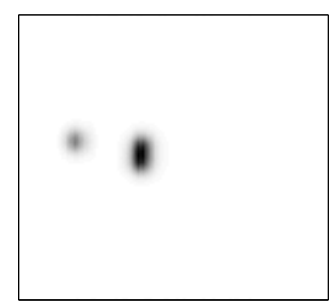

(e)

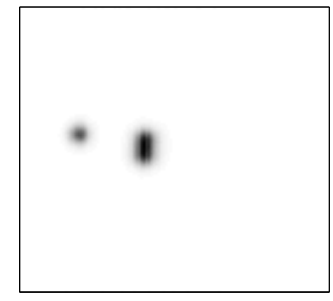

(h)

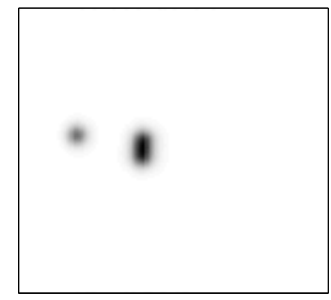

(k)

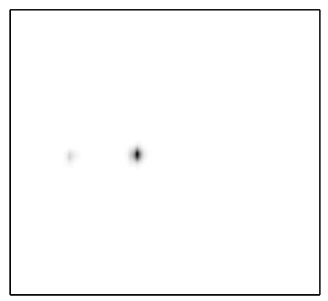

(c)

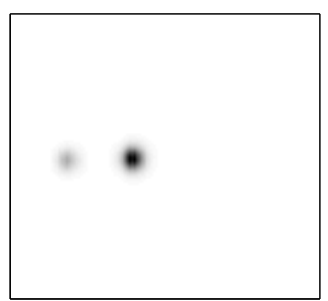

(f)

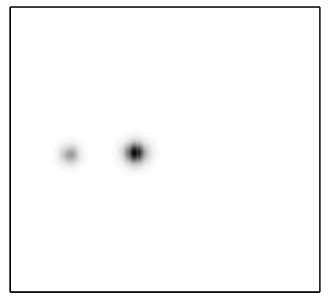

(i)

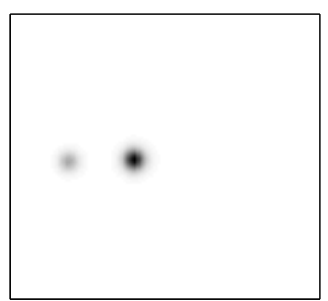

(l)

Figure 8.9: ${ }^{22} \mathrm{Na}$ phantom reconstructed from the separate datasets, using 100 Siddon lines. Images a), b) and c) are reconstructions of the Si-Si data, and are sums over the transverse, coronal and sagittal planes respectively. The corresponding images for Si-BGO are shown in d), e), f) for one side, in g), h), i) for the other and in j), k), l) for the combination of the two. 


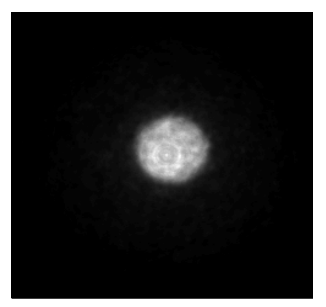

(a)

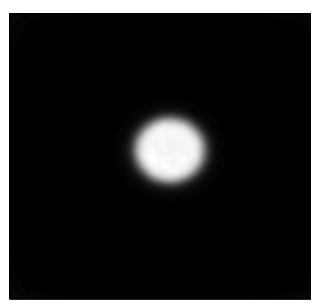

(d)

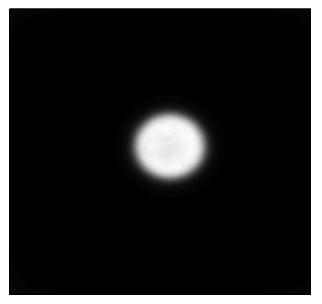

(g)

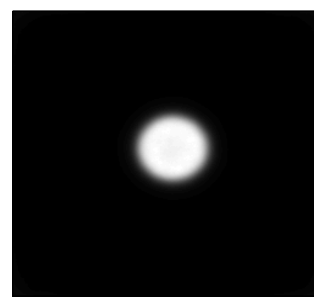

(j)

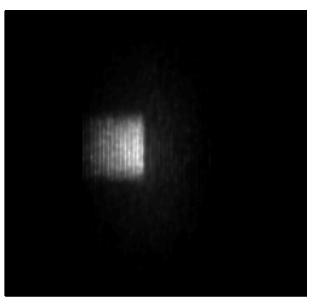

(b)

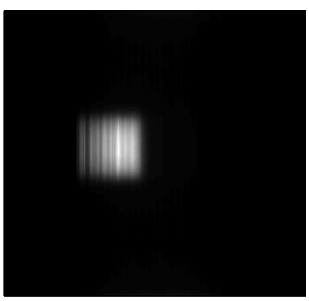

(e)

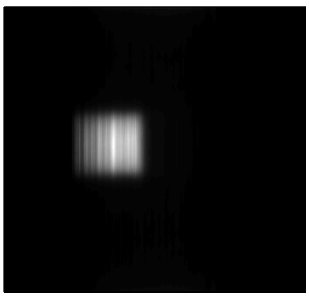

(h)

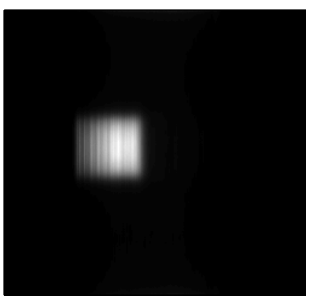

(k)

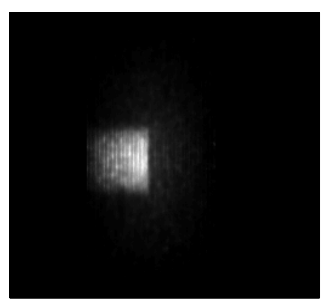

(c)

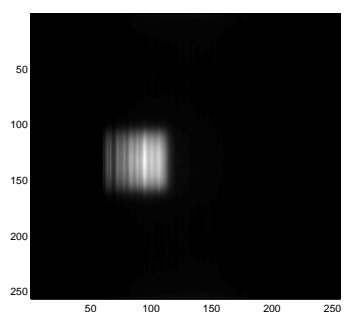

(f)

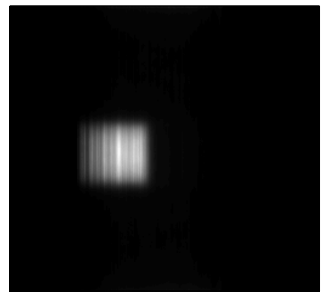

(i)

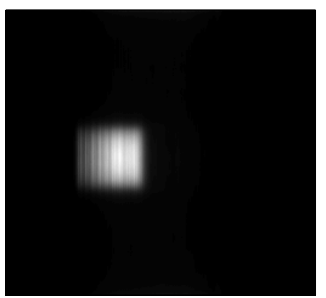

(1)

Figure 8.10: FDG-filled vile reconstructed from the separate datasets, using 100 Siddon lines. Images a), b) and c) are reconstructions of the Si-Si data, and are sums over the transverse, coronal and sagittal planes respectively. The corresponding images for Si-BGO are shown in d), e), f) for one side, in g), h), i) for the other and in j), k), l) for the combination of the two. 


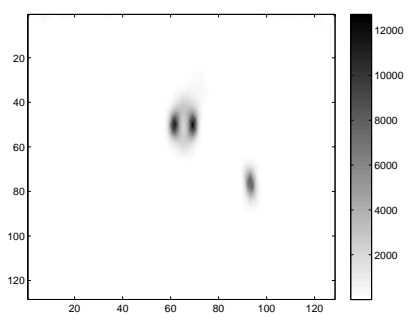

(a)

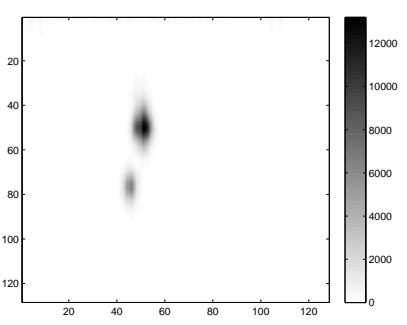

(b)

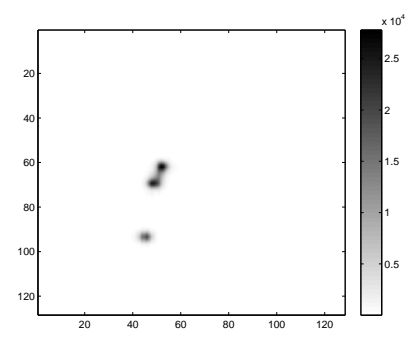

(c)

Figure 8.11: Images reconstructed from Si-BGO data in the 3D probe configuration. Summed slices of three orthogonal planes are shown: a) the transaxial, b) and c) two axial planes. The summed slices in c) are what is seen from the probe's point of view. The images correspond to the projections described in figure 8.5. 


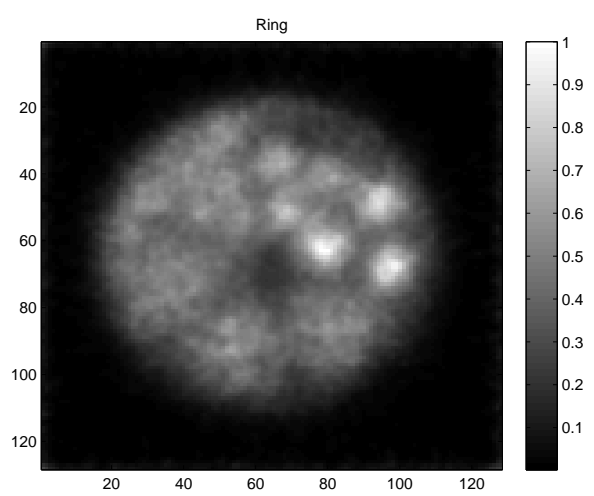

(a)

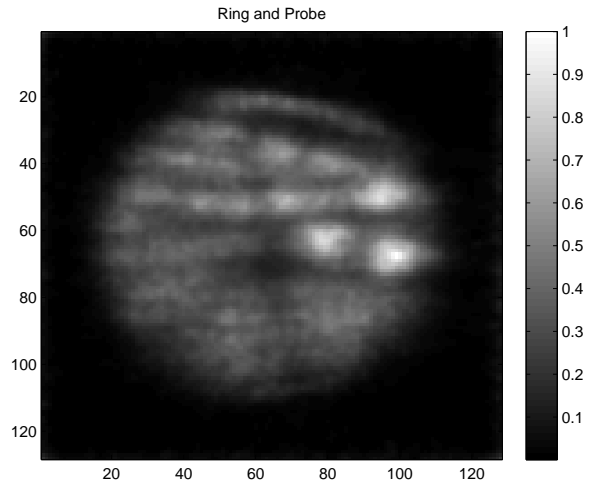

(b)

Figure 8.12: Images of the resolution phantom from the 2D probe data. Shown are images reconstructed using a) ring (BGO-BGO) data only and b) including probe data. The Si detector is to the right of the image. 


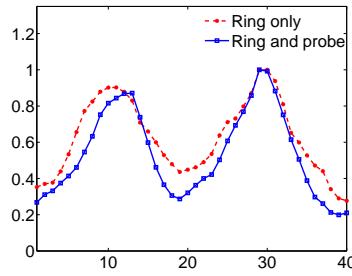

(a)

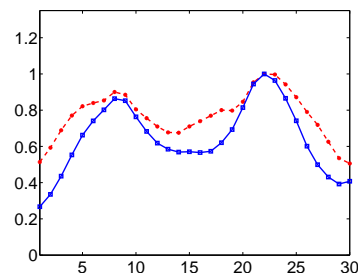

(d)

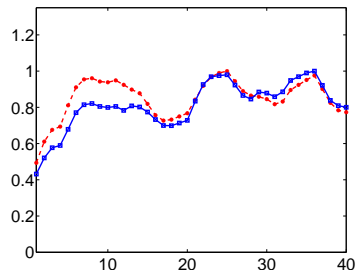

(g)

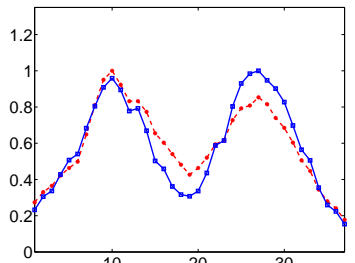

(b)

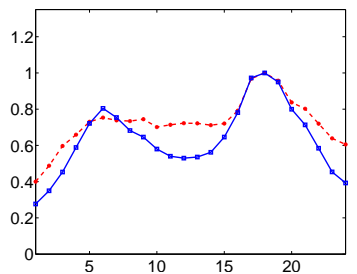

(e)

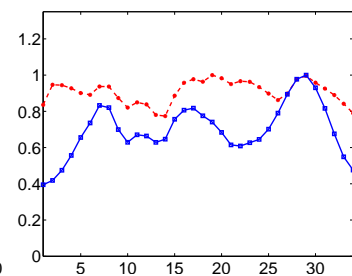

(h)

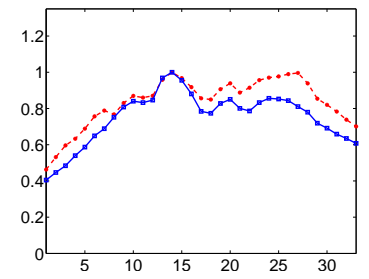

(j)

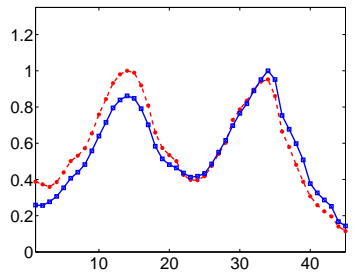

(c)

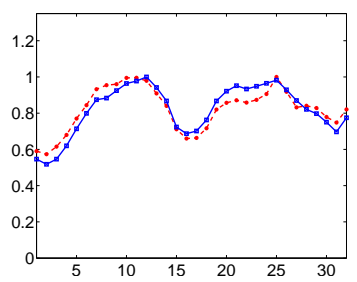

(f)

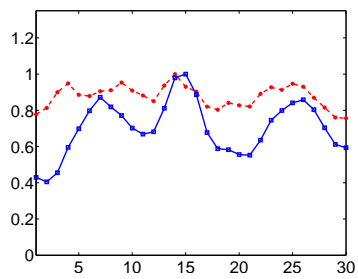

(i)

Figure 8.13: Comparison of profiles through the resolution phantom as defined in figure 8.6. Red lines represent the ring-only image and blue the one where probe data is included. 


\section{Part V}

\section{CONCLUSIONS}




\section{Conclusions and Prospects}

This chapter presents a summary of our contribution to the development of a high-resolution Positron Emission Tomography (PET) system, which utilizes a silicon $(\mathrm{Si})$ detector probe to obtain improved image quality in a limited region of interest (ROI). The following is an overview of the system under investigation and the image reconstruction method developed for it. Results of simulation studies into the effect that the Si probe would have on the image quality of a clinical PET scanner are summarized. The chapter concludes with a discussion of an experimental prototype of the system and prospects for future work.

PET is an invaluable clinical tool when used in combination with CT or MRI. However, compared to these other modalities, it has relatively low spatial resolution. Modern PET scanners achieve resolutions of $5 \mathrm{~mm}$ FWHM, while clinical CT and MRI are capable of sub-millimeter resolution. One of the methods proposed for improving the spatial resolution of PET is the combination of a clinical scanner with high-resolution detectors within the field of view (FOV). This approach has the benefit of utilizing pre-existing clinical devices. The simplest implementation of this technique is to use a single detector, or a compact group, in the form of a detector probe. Because of their high spatial and energy resolution, Si detectors were determined as an appropriate choice for the probe device. A prototype of the probe has 211 
been constructed by the CIMA group, a collaboration of five leading institutions, and used for several Si PET geometries. The geometry used in simulation studies combined a layered Si detector probe axially centred in the FOV of a clinical PET scanner based on the Siemens Biograph64. The Monte-Carlo package GATE was used for simulations. The use of GATE for simulating an insert geometry was a novel application of the Monte-Carlo package, which was developed with more conventional geometries in mind. It was shown that a device like the probe, which does not belong to the typical hierarchal organization of the PET scanner, can also be simulated.

The first main contributions of this work was the development of an image reconstruction software package suitable for reconstructing the multi-resolution data of the PET probe system and other geometries involving high resolution inserts. The package remains the only one capable of reconstructing fully 3D images from the CIMA Si-PET prototypes, although further modifications such as accurate sensitivity and attenuation corrections would be required for quantitative studies. The method was based on the Maximum Likelihood-Expectation Maximization (ML-EM) algorithm. The Si probe geometry required a dedicated implementation of the algorithm to suit its characteristic properties. The abundance of possible detector pairs in the data and the variable resolution of the possible lines of response (LORs), made sinograms and LOR histograms inefficient for use as the data input into the ML-EM equation. List-Mode ML-EM was considered to be most appropriate. System matrix elements were calculated on-the-fly due to the large number of possible LORs. The Siddon ray-tracing algorithm was used as an efficient method for this purpose, as well as for sensitivity matrix calculation. Multiple Siddon lines were required to accurately model low-resolution PET data in an image space of adequate granularity for high-resolution probe events. Attenuation, 
scatter and random coincidences were accounted for during reconstruction.

A special weighting factor was necessary in order to account for the difference in sensitivity of the system to the two types of events. An efficient method for calculating this factor for each probe position was presented. However, the method is sensitive to the effects of pile-up and dead-time on counting statistics, the effects of which require detailed investigation which is left to future work. Due to the multi-ray approach taken, additional advancements are required to reduce the current computational burden of the image reconstruction software. The aim of the current software was to provide a flexible and accurate reconstruction package, therefore little time was spent on optimization.

The second main contribution presented in this work is the quantification of the effect of the probe on the image quality obtained by the simulated PET scanner, as measured using a number of figures of merit. Comparison studies were conducted between the original PET system and the novel PET-probe geometry in terms of resolution, contrast, spill-over ratio (SOR) and contrast recovery coefficient (CRC). Resolution improvement was predicted and confirmed to be directionally dependent. In a region near to the probe, extending out to nearly $6 \mathrm{~cm}$ away from its surface, resolution was shown to increase in all directions, with improvements of up to $50 \%$ observed. Resolution enhancement was shown to extend out up to $20 \mathrm{~cm}$ from the probe surface, in both the axial and transaxial direction. Improvement in the direction perpendicular to the probe's surface was observed to be limited to the $6-\mathrm{cm}$ region. The results of the resolution studies indicate a ROI where resolution improvement can be expected. Such information can be useful for task-specific probe placement.

It has been shown that, while constituting only a moderate percentage of the total (1-30\%, depending on activity distribution), probe 
data was able to improve the resolution of the scanner. This observation indicates that, despite the low sensitivity of the Si detectors, their high spatial resolution has a notable effect on image resolution, making Si an appropriate material for this application.

It was demonstrated that the improvement in resolution did not come at the cost of other image quality measures. Small improvements in contrast and SOR were observed at various distances away from the probe surface. Close to the probe, an improvement of as high as $17 \%$ was observed. Measurements of CRC in a breast phantom containing spherical lesions, with diameters of 4, 5, 6 and $7 \mathrm{~mm}$, demonstrated how this quantity is improved in a region close to the probe surface. Improvements in CRC, which is correlated to lesion detectability, indicated that the addition of the probe would offer the advantage in small lesion detection. We must mention that the improvements to these image quality metrics did come at the expense of an artifact. A dip in the image intensity of a homogeneous region directly adjacent to the probe face, as observed during the breast phantom study, is likely due to a deficiency in the system model and possibly related to sensitivity. This should be the subject of further study.

Results from studies of an experimental prototype have been included in this thesis as an appendix and further support the results of the simulation studies. In particular, these demonstrate the high resolution capabilities of the PET probe system in the laboratory in a physical experiment. Images of a resolution phantom from the experimental PET scanner were compared to those which were obtained using the scanner and the probe prototype. The increase in resolution was evident, and the directionality of the improvement corresponded to that predicted by simulation studies. However, the limited spatial orientation of this improvement gave an oval appearance to circular phantom features, an artifact which may be misleading in a clinical setting.

The investigation into the PET-probe system has also revealed a 
number of specific challenges that should be addressed before such a system could be clinically employed. The main challenge of the present system is the poor timing-resolution of the Si detectors. The simulated resolution of $10 \mathrm{~ns}$ requires a time coincidence window of $20 \mathrm{~ns}$, which, at high activities, causes random fractions of over $90 \%$ in the probering data. The solution to this problem could come from hardware enhancements of the system and entail, for example, creating thinner $\mathrm{Si}$ detectors which would reduce depth-related broadening in the timing response. A double time-window has also been envisioned and could be implemented for use in future coincidence sorting. The observation that a significant fraction of random events occurs in LORs unrelated to phantom position, motivates the imposition of geometric acceptance limits in probe measurement space.

Further software improvements could also be made. A more accurate system model could be developed including effects such as depth of interaction (DOI) and attenuation in the block detectors, and a analytical method for estimating scatter would be beneficial. An analytic model of the sensitivity weighting factor should also be the subject of future work. A more sophisticated system model may further improve the reconstructed images and increase the benefit provided by the probe. The source of the intensity dip which was observed in the breast phantom studies should be studied and the effect included in system modeling. The apparent elongation of phantom features may be addressed by using multiple probe positions in one scan, a possibility which is also left to future investigation. In order to shorten reconstruction time, an Ordered Subsets Expectation Maximization algorithm is under development and testing for further investigation. This more efficient software should be further developed.

Further work can also be performed in exploring the capabilities of the probe system. Although resolution has been measured as a 
function of position relative to the probe, this was done for one probe location only. Varying the probe position would offer further insight into how the device could be used to modify the scanner's resolution, thus providing further information for task-specific probe positioning.

The probe has been shown to have the potential to improve the performance of a conventional PET scanner in a limited region of interest (ROI). By providing augmented resolution and lesion detection capabilities it is a practical way of upgrading an already-existing clinical device for applications where a "closer look" at a specific region is desired. The investigations described in this thesis into the properties of such a system, using a Si detector probe and a clinical PET scanner, have shed light on the advantages it provides. The modifications and extensions described can enhance the present work, developing the Si PET probe into a potentially usefully clinical tool. 


\section{Part VI}

\section{SUMMARY IN SPANISH}




\section{Estudio de un Sistema PET de Alta Resolución usando una Sonda de Detectores de Silicio}

\section{Introducción}

La tomografía por emisión de positrones (PET, en sus siglas en inglés) es ampliamente usada en la diagnosis clínica y en estudios pre-clínicos. Como herramienta diagnóstica, se usa principalmente en oncología, cardiología y neurología. Proporcionando información funcional en vez de estructural, PET es complementario a otras técnicas de imagen médica. Los escáneres PET modernos se combinan con aparatos de tomografía axial computarizada (TAC) o de imágen por resonancia magnética (MRI, en sus siglas en inglés), produciendo imágenes PET/TAC y PET/MRI, que combinan información estructural y metabólica en la misma imagen.

Sin embargo, una de las principales limitaciones de PET es su baja resolución espacial. Los escáneres clínicos típicamente tienen una resolución de $5 \mathrm{~mm}$ [1] (anchura a media altura, o FWHM por sus siglas 
en inglés) en el centro del compo de visión, mientras que imágenes TAC e IRM alcanzan una resolución de $1 \mathrm{~mm}$, aproximádamente.

Esta tesis presenta un estudio de un sistema PET de alta resolución, empleando el novedoso principio que combina un escáner convencional con una sonda de detectores de silicio, situada dentro del campo de visión del escáner.

Un detector de silicio ( $\mathrm{Si}$ ) de alta resolución, en coincidencia con un escáner clínico, debería producir imágenes de más alta calidad que las que se obtenienen usando solamente datos del escáner. La mejora en resolución espacial de la imágen, que aumentaría gracias al pequeño tamaño de los pixeles del detector, se limitaría a una región próxima a la sonda. El sistema PET desarrollado en este trabajo utiliza estos principios. Se consideraría una herramienta apropiada para estudios localizados como, por ejemplo, la diagnosis de cáncer de mama.

Los estudios presentados en este trabajo cuantifican el beneficio aportado por la sonda y la posible degradación de la imágen, e indican una posible aplicación clínica para el dispositivo. El sistema ha sido estudiado mediante simulaciones Monte Carlo y experimentos con un prototipo.

\section{Metodología}

La tesis se estructura en cuatro partes. En primer lugar, se diseñó un sistema que combina un anillo PET con una sonda de detectores de silicio, basandose en trabajos previamente realizados con geometrías parecidas. Las características del escaner clínico que sirvió de modelo para el sistema anillo-sonda y las propiedades de los detectores de silicio empleadas en la construcción del prototipo, se usaron para implementar una simulación realista del sistema. Segundo, se desarrollaron herramientas para la reconstrucción de la imágen a partir de los datos 
simulados. Debido a su novedosa geometría, métodos convencionales de reconstrucción no se podían aplicar al sistema anillo-sonda. Un software dedicado se implementó, con la flexibilidad necesaria para su uso tanto con datos simulados como con los obtenidos con el sistema prototipo. En tercer lugar, se estudiaron los efectos de la sonda sobre las imágenes reconstruidas. Se investigó la resolución espacial, el contraste y la detectabilidad de lesiones en el ámbito de imágenes de mama. Por último, se obtuvieron imágenes usando un prototipo experimental del sistema.

\section{El sistema anillo-sonda}

El escaner clínico en el que se basa el componente anillo del sistema anillo-sonda es el Biograph64, comercializado por Siemens ${ }^{1}$. El Biograph consiste en detectores de cristales centelladores (LSO) con dimensiones $4 \times 4 \times 20 \mathrm{~mm}^{3}$, organizados en bloques de $13 \times 13$. El escáner tiene un diametro de $842 \mathrm{~mm}$ y extensión axial de $162 \mathrm{~mm}$. La sonda está formada por 10 capas de detectores de silicio, de $1 \mathrm{~mm}$ de grosor y separadas por $1 \mathrm{~mm}$, con pixeles de una superficie de $1 \times 1 \mathrm{~mm}^{2}$. La figura 14 presenta la geometría, con la sonda junto con un fantoma Derenzo.

El sistema se simuló empleando el paquete Monte Carlo GATE [2]. En los detectores del escáner se simuló una resolución temporal de 2.25 ns FWHM, y una resolución energética de $15 \%$ a $511 \mathrm{keV}$, siguiendo las especificaciones del fabricante. En la sonda se usó una resolución temporal de 10 ns FWHM y una resolución energética constante (independiente de la energía del fotón) de $1.5 \mathrm{keV}$ FWHM, valores basados en medidas hechas con un prototipo de la sonda $[3,4,5]$. Para formar coincidencias se usó una ventana energética de 400-650 keV, la del Biograph. Para la ventana temporal, se usó 4.5 ns

\footnotetext{
${ }^{1}$ activexray.com/pdf/Siemens ${ }^{*}$ Biograph.pdf
} 


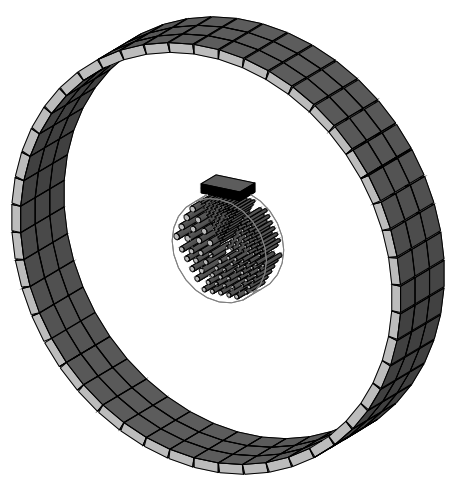

(a)

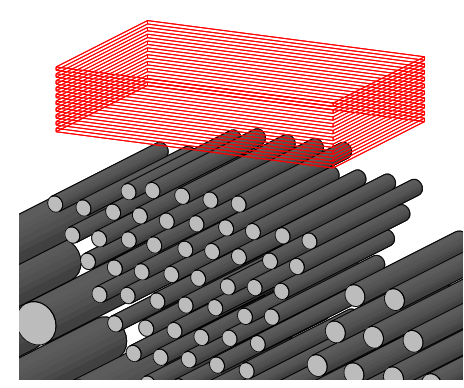

(b)

Figura 14: El sistema PET con la sonda de silicio cerca de la superficie de un fantoma Derenzo. En a) se ve el sistema entero y en b) la sonda en primer plano. Las capas de la sonda se visualizan como capas transparente y no se muestran los píxeles individuales.

para formar coincidencias del sistema PET convencional (sin sonda), según la práctica común de escoger el doble de la resolución temporal de los detectores. Para el sistema anillo-sonda, las coincidencias se formaron usando el mismo principio, pero usando una ventana temporal de 20 ns, igual a doble la resolución de los detectores de Si, para mayor sensibilidad. Un umbral energético de $20 \mathrm{eV}$ se implementó en la sonda, representativo de del valor que se usaría para reducir el ruido en la señal del silicio.

\section{Reconstrucción de la imagen}

Para la reconstrucción de imagen, en este trabajo se ha utilizado el algoritmo ML-EM (Maximum Likelihood Expectation Maximization, en inglés) en modo lista (List-Mode ML-EM). Con este algoritmo iterativo, la imágen $\boldsymbol{f}$ obtenida en la iteración $k+1$ se calcula usando: 


$$
f_{j}^{k+1}=f_{j}^{k} \sum_{i=1}^{M} a_{i j} \frac{1}{\sum_{j=1}^{J} a_{i j} f_{j}^{k}} .
$$

En esta expresión, $\boldsymbol{a}$ es la llamada matriz de sistema, cuyo elemento $a_{i j}$ es proprcional a la probabilidad de que un evento detectado y con origen en el pixel $j$ de la imagen, se haya detectado por el par de detectores que corresponden al evento $i$. Este algoritmo básico se adoptó a las características especificas del sistema sonda-anillo.

Se han aplicado correcciones por coincidencias aleatórias y dispersadas en el fantoma se han aplicado mediante una modificación en el denominador de la ecuasión 2 según:

$$
\sum_{j=1}^{J} a_{i j} n_{j}^{k}+r_{i}+s_{i}
$$

El total de coincidencias aleatorias, $r_{i}$, en el par de detectores $i$ se calculó a partir de la tasa estimada de estos eventos, $R_{i}$, y el tiempo de adqusición. La estimación de la tasa se obtuvo empleando la ecuación

$$
R_{i}=2 \tau S_{i_{1}} S_{i_{2}},
$$

donde $\tau$ es la magnitud de la ventana temporal y $S_{i_{1}}$ y $S_{i_{2}}$ son las tasas de cuentas en los dos detectores individuales. El total de coincidencias con dispersión en el fantoma $s_{i}$ se calculó usando un método Monte Carlo.

\section{Calidad de imagen}

Para evaluar el beneficio que la sonda aporta a la calidad de la imágen, se han hecho varias simulaciones del sistema con diversos fantomas. Estudios de resolución espacial, contraste y detectabilidad de lesiones se han hecho con fantomas dedicados. En cada caso, se ha simulado el sistema PET convencional y el sistem anillo-sonda por separado. 


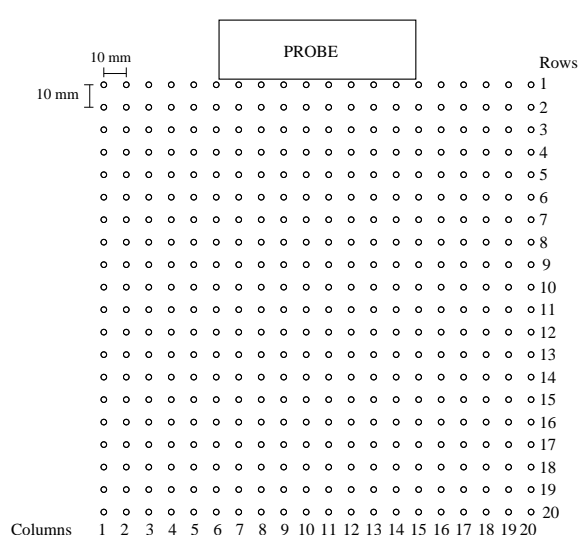

Figura 15: La matriz de posiciones donde se midió la PSF.

Resolución espacial. Para estudiar la resolución espacial, se ha simulado una matriz (en 2D) de fuentes puntuales en el plano transaxial central del campo de visión del escaner, sobre un fondo cuadrado homogéneo, relleno de agua. Las fuentes estaban separadas por $10 \mathrm{~mm}$ en ambas direcciones y contenían una actividad acumulada de $10 \mu \mathrm{Ci}$ mientras que el fondo contenía $1 \mathrm{mCi}$. Imágenes de los dos sistemas se reconstruyeron usando 20 iteraciones del ML-EM, previamente establecidas como suficientes para conseguir la convergencia de resolución. Se restó el fondo homogéneo de las imágenes reconstruídas y se midió la point-spread function (PSF) en 3 direcciones perpendiculares. La figura 15 demuestra la posición relativa de los puntos a la sonda y una numeración para indentificarlos en el momento de presentar los resultados.

Contraste y SOR. El efecto de la sonda sobre el contraste y la spill-over ratio (SOR) se midió usando un fantoma conteniendo lesiones esféricas de $16 \mathrm{~mm}$ de diámetro, calientes y frías y a varias distancias de la sonda, sobre un fondo "templado" homogéneo. No 


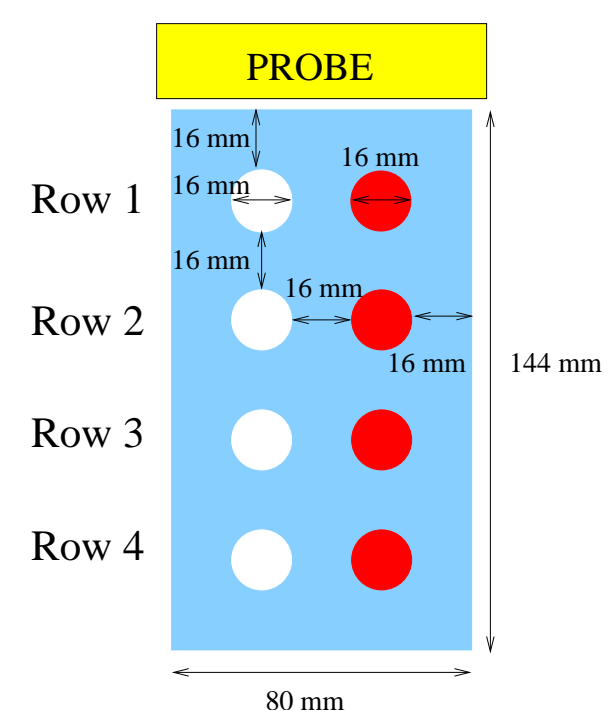

Figura 16: El fantoma usado en estudios de contraste y SOR, en su posición relativa a la sonda. El fantoma está centrado en el FOV. Las filas 1, 2, 3 y 4 corresponden a distancias de 27, 59, 91 y $123 \mathrm{~mm}$.

se ha simulado ningún medio de dispersión. La figura 16 ilustra el fantoma en su posisión relativa a la sonda.

Imágenes de los dos sistemas se reconstruyeron unsando 50 iteraciones del ML-EM. Regiones de interes (Regions Of Interest, ROIs) se elegieron usando esferas de $12 \mathrm{~mm}$ de diámetro, centradas sobre cada uno de las lesiones y en el espacio entre cada par frío/caliente. Las ROIs centrales entre cada par sirvieron de regiones templados. El contraste caliente-templado $\left(C_{\text {hot } / \text { warm }}\right)$, templado-frío $\left(C_{\text {warm/cold }}\right)$ y es SOR se midieron según las siguientes definiciones:

$$
S O R=\frac{\mu_{\text {cold }}}{\mu_{\text {warm }}},
$$




$$
\begin{gathered}
C_{\text {hot } / \text { warm }}=\frac{\mu_{\text {hot }}-\mu_{\text {warm }}}{\mu_{\text {warm }}}, \\
C_{\text {warm } / \text { cold }}=\frac{\mu_{\text {warm }}-\mu_{\text {cold }}}{\mu_{\text {warm }}} .
\end{gathered}
$$

En las ecuaciones $\mu$ simboliza el valor medio de cada uno de los ROIs.

\section{Aplicación a mamografía PET}

Para investigar el efecto de la sonda sobre la detectabilidad de lesiones y demostrar su aplicación a escaneados mamarios, se simuló un fantoma antropomorfo relleno de agua, consistiendo de un torso, un corazón y una mama con lesiones de diferentes tamaños. La geometría de la simulación está ilustrada en la figura 17.

\section{Prototipo experimental}

Se ha estudiado el principio de la sonda de Si también en el laboratorio, empleando un prototipo del sistema anillo-sonda, en 2D. La geometría consistía en dos anillos parciales (o arcos) de detectores centelladores, con una plataforma giratoria al centro, donde se colocaba el fantoma. Una rotación completa de la plataforma permitía la colección de las proyecciones necesarias para formar una imágen tomográfica. Colocando la sonda de Si entre uno de los arcos y el fantoma, ahora sin rotar este último, se obtienen coincidencias entre la sonda y el arco opuesto. Los datos obtenidos se limitaron a un solo plano mediante un sistema de colimación de plomo. Una fotografía muestra el prototipo en la figura 18. 


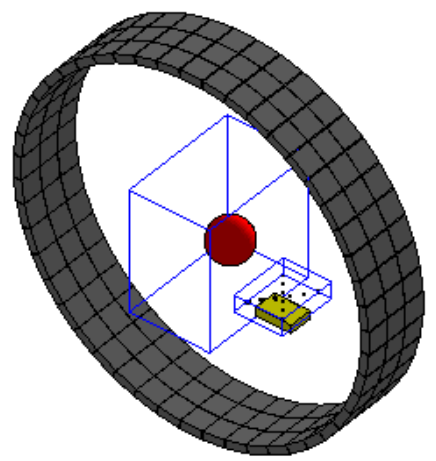

(a)

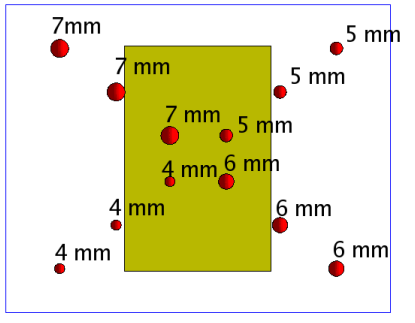

(b)

Figura 17: El fantoma antropomorfo en y posición relativa al escaner y la sonda. En a), el torso y la mama son transparentes, el corazón un esfera y la sonda está situada por debajo de la mama. En b) se demuestra la distribución de las lesiones esféricas dentro de la mama, y su posición relativa a la sonda.

\section{Resultados}

En la figura 19 se presenta parte de los resultados del estudio de la resolución espacial. La resolución de los puntos de la columna 10 (column 10 según la figura 15), cuantificada por el FWHM, se presenta como función del numero de la fila (row, según la figura 15) que representa la distancia de la sonda. Los resultados del Biograph, enseñados junto con los del sistema anillo-sonda para comparar, demuestran el aumento de resolución aportado por la sonda.

Los resultados del estudio de contraste y SOR se muestran en la figura 20. Las gráficas demuestran la evolución de las tres figuras de mérito con el número de iteraciones usadas. Los valores teóricos de $\left(C_{\text {hot } / \text { warm }}\right),\left(C_{\text {warm/cold }}\right)$ y SOR son 4,1 y 0 , respetivamente. 


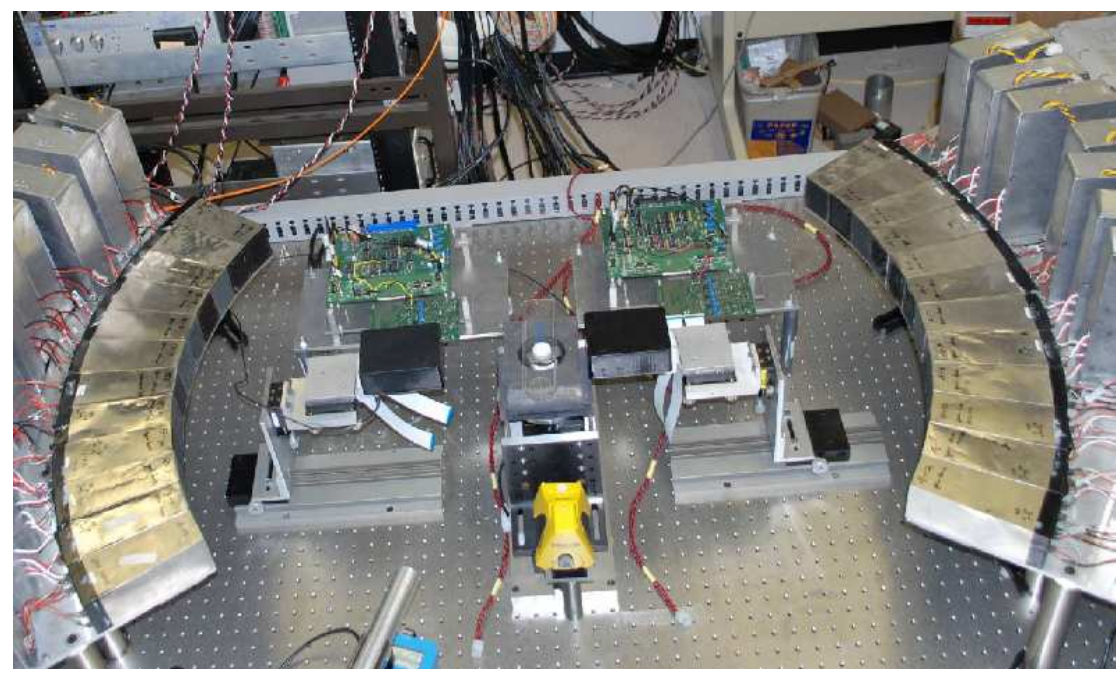

Figura 18: Fotografía de prototipo experemental. En el centro está visible el colimador de plomo que rodea el fantoma. Las dos cajas negras a cada lado del fantoma contienen dos sondas, de las cuales se usa una para la geometría anillo-sonda y las dos para un sistema doble-anillo.

Los resultados del estudio de detectabilidad de lesiones se muestran en la figura 21. Las imágenes enseñan solamente la parte del campo de visión donde se encuentran la mama y las lesiones.

Las imágenes del fantoma Derenzo, reconstruidas con datos del prototipo experimental del sistema se ven en la figura 22 , con y sin sonda.

\section{Discusión y Conclusiones}

Los resultados de los estudios realizados con datos simulados han demostrado una clara mejora en la calidad de las imágenes cuando se usa la sonda en combinación con el escáner clínico convencional. Se ha 


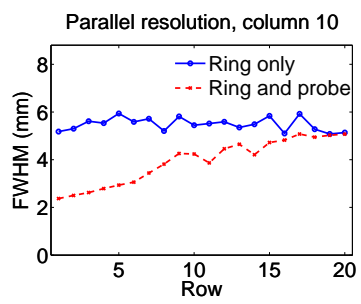

(a)

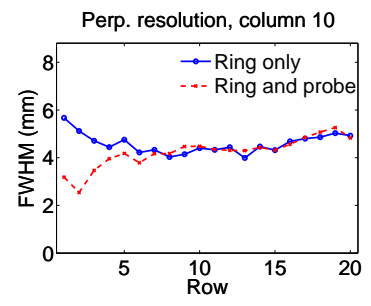

(b)

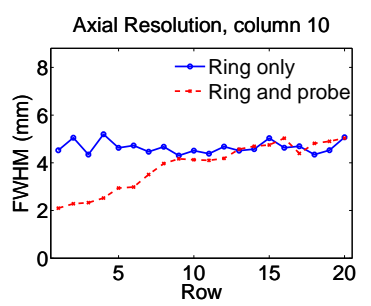

(c)

Figura 19: Comparación de la resolución espacial del Biograph y la del sistema sonda-anillo, usando datos de la columna central de la matriz de fuentes puntuales. La resolución transaxial se presenta en la dirección (a) paralela y (b) perpendicular a la superficie de la sonda. La resolución axial se muestra c). Los números de columnas (column) y filas (row) corresponden a posiciones en la figura 15 .

demostrado que la resolución espacial mejora en un factor dos cerca de la superficie de la sonda y este aumento se va reduciendo más lejos de la sonda a un ritmo que depende de la dirección en que está medida. El aumento de la resolución alcanza distancias de la sonda mayores cuando es medido en la dirección paralela a su superficie, sea ésta axial o transaxial. El estudio usando el fantoma Derenzo ha demonstrado claramente un aumento de resolución espacial.

El estudio de contraste y SOR demuestra que el aumento en resolución espacial que proporciona la sonda no conlleva una degradación de otros aspectos de la calidad de la imágen. El estudio tampoco in- 

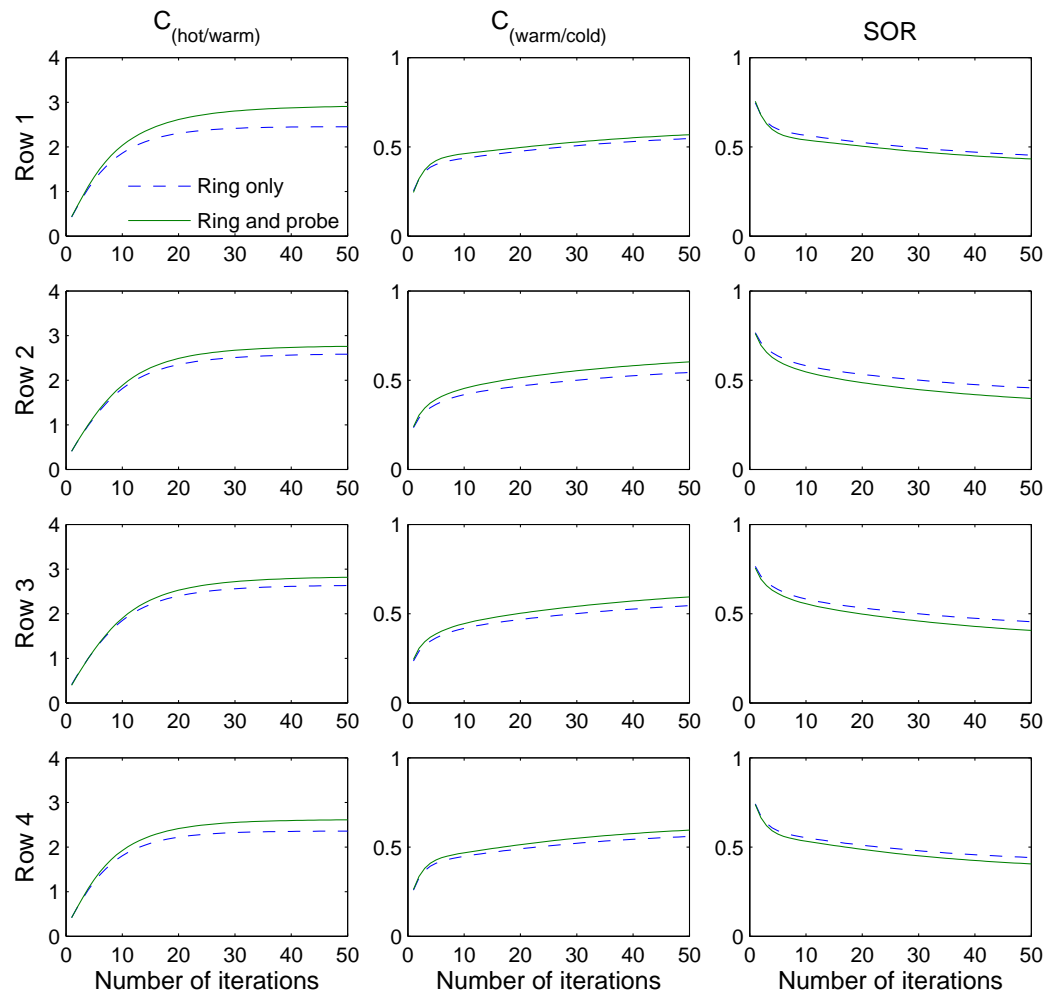

Figura 20: $C_{\text {hot } / \text { warm }}, C_{\text {warm/cold }}$ y SOR a varias distancias de la sonda en función de número de iteraciones. Las filas (rows) 1, 2, 3, 4 corresponden a distancias de 27, 59, 91 y $123 \mathrm{~mm}$. La linea sólida representa valores del sistema anillo-sonda mientras que la linea discontinua representa medidas de la simulación del Biograph sin sonda.

dica su mejora. Sin embargo, los tamaños de las lesiones que se han usado para el estudio eran suficientemente grandes como para asegurar un rendimiento adecuado del Biograph, en cual caso no se espera ver mucha mejora empleandon la sonda. 


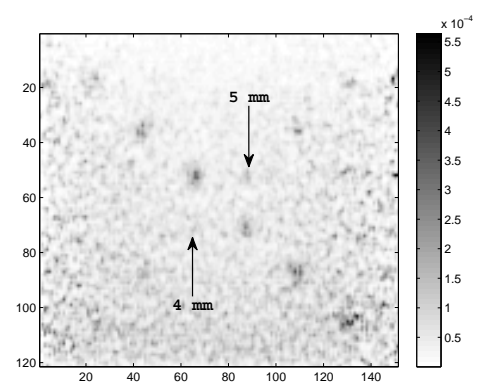

(a)

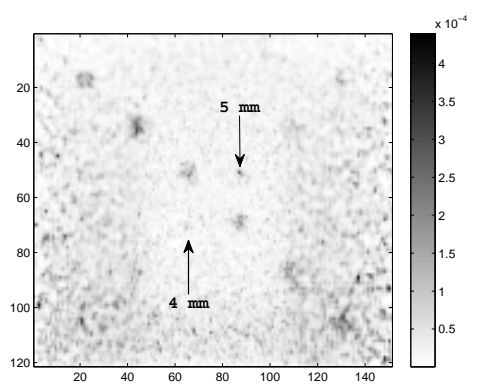

(b)

Figura 21: Imágen de la mama a) sin usar la sonda y b) incluyendo la sonda en la simulación. En las imágenes, las lesiones son vistas desde el lado opuesto al que se encuentra la sonda.

La sonda se ha demonstrado capaz de aumentar la detectabilidad de lesiones, en un estudio de una mamografía PET. En la figura 21, la lesión de $5 \mathrm{~mm}$ que se encuentra la más cercana a la superficie de la sonda se ve claramente mejor en la imágen donde se ha empleado la sonda. Además de las imágenes mostradas en este resumen, se ha hecho un estudio cuantitativo de detectabilidad de lesiones que ha sido excluído de este breve resumen. Las medidas de contrast-to-noise ratio (CNR) han demonstrado que la detectabilidad de las cuarto lesiones situadas arriba de la superficie de la sonda es aumentada por su uso $[6]$.

El principio de la sonda de silicio también ha sido demostrado usando un prototipo experimental del sistema sonda-anillo. Imagenes de un fantoma Derenzo, reconstruidas con datos obtenidos usando una sonda de silicio y un escáner PET convencional, demuestran un aumento en resolución espacial si son comparadas con imágenes que solamente usan datos del escáner. 


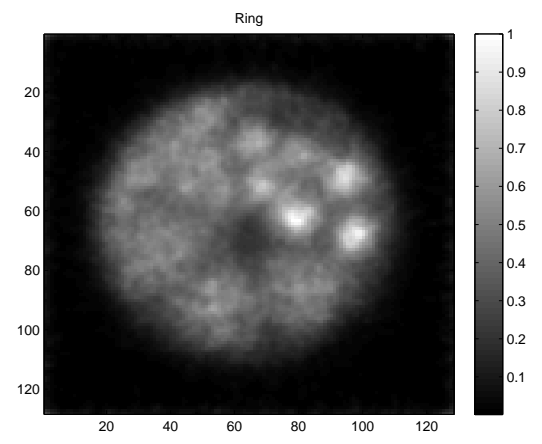

(a)

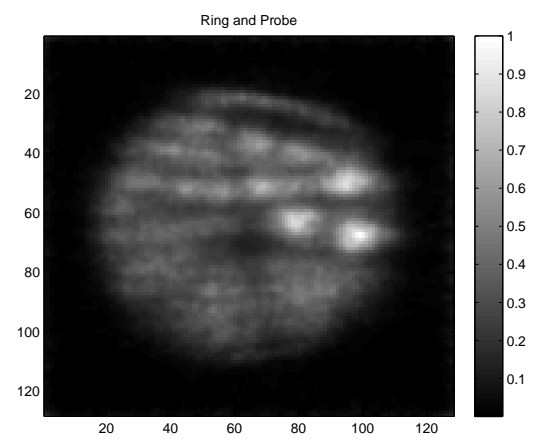

(b)

Figura 22: Imágenes del fantoma Derenzo reconstruidos con a) datos del anillo solamente y b) incluyendo datos de la sonda.

\section{Referencias}

[1] Lonsdale, M.N., Beyer, T.: Dual-modality PET/CT instrumentation - Today and tomorrow. European Journal of Radiology 73(3) (2010) $452-460$

[2] Jan, S., et al.: GATE: a simulation toolkit for PET and SPECT. Phys. Med. Biol. 49 (2004) 4543 
[3] Clinthorne, N., Burdette, D., Studen, A., Honscheid, K., Kagan, H., Chesi, E., Huh, S., Lacasta, C., Llosá, G., Mikuž, M., Smith, D., Weilhammer, P.: Timing in silicon pad detectors for compton cameras and high resolution PET. In: Nuclear Science Symposium Conference Record, 2005 IEEE. Volume 5. (Oct 2005) 2868-2872

[4] Studen, A., et al.: Performance of the MADEIRA PET probe prototype. In: IEEE Nucl. Sci. Symp. Med. Imaging Conf. (24 2009-nov. 1 2009) 3111

[5] Studen, A., Burdette, D., Chesi, E., Cindro, V., Clinthorne, N., Cochran, E., Grošičar, B., Honscheid, K., Kagan, H., Lacasta, C., Llosá, G., Linhart, V., Mikuž, M., Stankova, V., Weilhammer, Z., Žontar, D.: Performance of the MADEIRA PET probe prototype. In: Nuclear Science Symposium Conference Record (NSS/MIC), 2009 IEEE. (Oct 2009) 3111-3115

[6] Brzezinski, K., Oliver, J.F., J., G., Rafecas, M.: Study of a highresolution PET system using a silicon detector probe. Phys. Med. Biol 52 (2014) 2807 


\section{Appendix}

\section{Publications Associated with the Thesis}

[1] Grkovski, M., Brzezinski, K., Cindro, V., Clinthorne, N.H., Kagan, H., Lacasta, C., Mikuž, M., Solaz, C., Studen, A., Weilhammer, P., Žontar, D.: Evaluation of a high resolution silicon PET insert module. Nucl. Inst. Meth. Phys. Res. Sec. A 788 (2015) 86 - 94

[2] Brzezinski, K., Oliver, J.F., Gillam, J., Rafecas, M.: Study of a high-resolution PET system using a silicon detector probe. Phys. Med. Biol 52 (2014) 6117-6140

[3] Clinthorne, N.H., Brzezinski, K., Chesi, E., Cochran, E., Grošičar, B., Honscheid, K., Huh, S., Kagan, H., Lacasta, C., Linhart, V., Mikuž, M., Stankova, V., Studen, A., Weilhammer, P., Žontar, D.: Silicon as an unconventional detector in position emission tomography. Nucl. Inst. Meth. Phys. Res. Sec. A 699 (2013) 216220

[4] Studen, A., Brzezinski, K., Chesi, E., Cindro, V., Clinthorne, N.H., Cochran, E., Grošičar, B., Grkovski, M., Honscheid, K., Kagan, H., Lacasta, C., Llosá, G., Mikuž, M., Stankova, V., Weilhammer, P., Žontar, D.: Silicon detectors for combined MR-PET 
and MR-SPECT imaging. Nucl. Inst. Meth. Phys. Res. Sec. A 702 (2013) $88-90$

[5] Studen, A., Brzezinski, K., Chesi, E., Cindro, V., Clinthorne, N.H., Grkovski, M., Grosicar, B., Honscheid, K., Huh, S.S., Kagan, H., Lacasta, C., Llosá, G., Mikuž, M., Rudge, A., Smith, S., Stankova, V., Weilhammer, P., Žontar, D.: A laboratory PET scanner with silicon detectors segmented to $1 \mathrm{~mm}$ detection cells. In: Nuclear Science Symposium Conference Record, 2012 IEEE. (2012) 1305-1310

[6] Studen, A., Brzezinski, K., Chesi, E., Cindro, V., Clinthorne, N., Grkovski, M., Grošičar, B., Honscheid, K., Huh, S., Kagan, H., Lacasta, C., Llosá, G., Linhart, V., Mikuž, M., Stankova, V., Weilhammer, P., Žontar, D.: Narrowing coincidence timing window through time-walk correction in silicon ring enhanced PET scanner. In: Nuclear Science Symposium and Medical Imaging Conference (NSS/MIC), 2011 IEEE. (Oct 2011) 266-270

[7] Brzezinski, K., Gillam, J., Oliver, J.F., Lacasta, C., Rafecas, M.: Multiple Resolution PET Data: Image Properties using the ListMode MLEM Reconstruction Algorithm. In: 11th International Meeting on Fully Three-Dimensional Image Reconstruction in Radiology and Nuclear Medicine. (2011) 282-285

[8] Brzezinski, K., Oliver, J.F., Gillam, J., Lacasta, C., Rafecas, M.: Observing the high resolution capabilities of a silicon PET insert probe. In: Medical Imaging 2011: Physics of Medical Imaging. Volume 7961. (2011) 796146

[9] Brzezinski, K., Oliver, J.F., Llosá, G., Solevi, P., Linhart, V., Cabello, J., Lacasta, C., Rafecas, M.: Monte-Carlo modelling of 
a silicon detector insert combined with a PET scanner. In: 5th

European Molecular Imaging Meeting EMIM2010. (2010) 184 